\title{
Good night, sleep tight : performance and EEG measures in primary insomnia patients and during sleep deprivation in health volunteers
}

Citation for published version (APA):

Perrier, J. I. (2015). Good night, sleep tight : performance and EEG measures in primary insomnia patients and during sleep deprivation in health volunteers. [Doctoral Thesis, Maastricht University]. Datawyse / Universitaire Pers Maastricht. https://doi.org/10.26481/dis.20151118jp

Document status and date:

Published: 01/01/2015

DOI:

10.26481/dis.20151118jp

Document Version:

Publisher's PDF, also known as Version of record

Please check the document version of this publication:

- A submitted manuscript is the version of the article upon submission and before peer-review. There can be important differences between the submitted version and the official published version of record.

People interested in the research are advised to contact the author for the final version of the publication, or visit the DOI to the publisher's website.

- The final author version and the galley proof are versions of the publication after peer review.

- The final published version features the final layout of the paper including the volume, issue and page numbers.

Link to publication

\footnotetext{
General rights rights.

- You may freely distribute the URL identifying the publication in the public portal. please follow below link for the End User Agreement:

www.umlib.nl/taverne-license

Take down policy

If you believe that this document breaches copyright please contact us at:

repository@maastrichtuniversity.nl

providing details and we will investigate your claim.
}

Copyright and moral rights for the publications made accessible in the public portal are retained by the authors and/or other copyright owners and it is a condition of accessing publications that users recognise and abide by the legal requirements associated with these

- Users may download and print one copy of any publication from the public portal for the purpose of private study or research.

- You may not further distribute the material or use it for any profit-making activity or commercial gain

If the publication is distributed under the terms of Article $25 \mathrm{fa}$ of the Dutch Copyright Act, indicated by the "Taverne" license above, 


\section{Good night, sleep tight}

\section{Performance and EEG measures in primary insomnia patients and during sleep deprivation in healthy volunteers}


(C) Joy Perrier, 2015

All rights reserved. No part of this thesis may be reproduced or transmitted in any form or by any means without prior written permission of the author.

(C) Cover design: Michele Colombo, Amsterdam

Printed by: Datawyse - Universitaire Pers Maastricht

ISBN: 9789461594983 


\section{Good night, sleep tight \\ Performance and EEG measures in primary insomnia patients and during sleep deprivation in healthy volunteers}

\section{DISSERTATION}

To obtain the double degree of Doctor at the Maastricht University and at the Caen - Normandie University

on the authority of the Rector Magnificus of the Maatricht University, Prof. dr. L.L.G. Soete and of the President of the Caen - Normandie University, Prof. P.

Sineux

in accordance with the decision of the Board of Deans,

to be defended in public on the $18^{\text {th }}$ of November 2015 , at 14:00 hours

by

Joy Irène Francine Perrier 


\section{Promotor:}

Prof.dr. J.G. Ramaekers

\section{Copromotor:}

Dr. M.L. Bocca (Université de Caen -Normandie)

\section{Assessment Committee:}

Prof. Dr W. J. Riedel (Chair)

Dr. A. Blokland

Prof. dr. M. Boulouard (Université de Caen - Normandie)

Prof. dr. K. Brookhuis (Rijksuniversiteit Groningen)

Dr. G. Rauchs (INSERM - Université de Caen - Normandie)

Prof. dr. E. J. W. van Someren (Universiteit van Amsterdam)

The research described in this dissertation was conducted as part of a double degree at the Unit COMETE U1075, University of Caen Basse Normandie, France (Chapters 2, 3;6) and the department of Neuropsychology and Psychopharmacology, Faculty of Psychology and Neuroscience, Maastricht University, The Netherlands (Chapters 4 and 5). Studies 2-4 were funded by the project DRiving Under the Influence of Drugs (DRUID), which was part of the European Union's 6th Framework Program. The research described here reflects only the author's view. The European Community is not liable for any use that may be made of the information contained therein. 


\section{Université de Caen Normandie}

Ecole doctorale Normande de Biologie Integrative, Santé et Environnement (EDNBise)

\section{Thèse de doctorat}

présentée et soutenue le : 18/11/2015 à $14: 00$ heures

par

\section{Joy Perrier}

pour obtenir le

\section{Doctorat de l'Université de Caen Normandie}

Spécialité : Biologie des populations et des organismes

préparée dans le cadre d'une cotutelle internationale de thèse entre l'Université de Caen Normandie et l’Université de Maastricht

\section{Performances diurnes et fluctuations du signal EEG chez des patients souffrant d'insomnie primaire et chez des participants sains en condition de privation de sommeil}

\section{Jury}

Directrice de thèse : Dr. Marie Laure Bocca

E. J. W. van Someren, Professeur des universités, Universiteit van Amsterdam, Rapporteur

K. Brookhuis, Professeur des universités, Rijksuniversiteit Groningen, Rapporteur

W. J. Riedel, Professeur des universités, Maastricht University

M. Boulouard, Professeur des universités, Université de Caen - Normandie

A. Blokland, Maître de conférence, Maastricht University

G. Rauchs, Chargée de recherche, Institut National de la Santé et de la Recherche Médicale

J.G. Ramaekers, Professeur des universités, Maastricht University

M.L. Bocca, Maitre de conférence, Habilitée à diriger des recherches, Université de Caen Normandie 

Paranimfen

Anne Havermans Stefan Jongen 


\section{Tables of contents}

CHAPTER 1

GENERAL INTRODUCTION

ATTENTION AND VIGILANCE DEFICITS IN PRIMARY INSOMNIA

ChAPTER 2

ATTENTIONAL NETWORKS AND VIGILANCE IN PRIMARY INSOMNIA

CHAPTER 3

IMPAIRED DRIVING PERFORMANCE ASSOCIATED WITH EFFECT OF TIME DURATION IN PATIENTS WITH PRIMARY INSOMNIA.

CORTICAL ACTIVITY MODIFICATIONS IN PRIMARY INSOMNIA AND AFTER SLEEP DEPRIVATION

CHAPTER 4

ELECTROENCEPHALOGRAPHY DURING ON-THE-ROAD DRIVING IN OLDER UNTREATED INSOMNIA PATIENTS AND NORMAL SLEEPERS

CHAPTER 5

DRIVING PERFORMANCE AND EEG FLUCTUATIONS DURING ON-THE-ROAD DRIVING

FOLLOWING SLEEP DEPRIVATION.

CHAPTER 6

SPECIFIC ELECTROENCEPHALOGRAPHIC SLEEP PATTERN IN THE PREFRONTAL CORTEX IN PRIMARY INSOMNIA: A SPECTRAL ANALYSIS

Chapter 7

GENERAL DISCUSSION

REFERENCES

SUMMARY

175

RESUME

181

VALORIZATION ADDENDUM

189

REMERCIEMENTS/ACKNOWLEDGMENTS

201

CURRICULUM VitAE

205

PUBLICATIONS LIST 
Chapter 1

General introduction 


\section{Epidemiology of sleep deprivation and insomnia}

Sleep disruption has become a major problem in Western society $(1,2)$. Among the different types of sleep disruption, we will consider sleep deprivation and chronic insomnia in the current dissertation. The latter is one of the most prevalent health concerns $(3,4)$. Between 10 and $15 \%$ of adults in the general population is reported to have complaints of insomnia associated with daytime consequences, and between 6 and 10\% meet the Diagnostic Statistical Manual of Mental Disorders, $4^{\text {th }}$ Edition (DSMIV) criteria for insomnia (5-7). According to the DSM-IV definition, primary insomnia (PI) is a complaint of non restorative and/or insufficient sleep associated with complaints of daytime consequences without comorbidity. Using this definition, the prevalence of PI was found to occur in $3-5 \%$ of the general population (7).

Both insomnia and sleep deprivation can lead to a range of adverse consequences on daytime perf ormance, health and productivity (8-14) that place a heavy economic burden on our society (14-17). It has been estimated that, in the U.S., insomnia was associated with $7.2 \%$ of all costly workplace accidents and errors (18), and the Sleep Health Foundation evaluated the indirect costs of sleep loss at 1.5 billion dollars in 2011 (14). Such cognitive and psychomotor dysfunction may hamper day-to-day operations such as driving a car. Indeed, insomnia has been indicated as an additional risk factor for traffic accidents (1921). Epidemiological studies have also shown that sleep deprivation leads to increased sleepiness and increased risk of traffic accidents (22-25). Furthermore, crash risk following sleep deprivation seems most pronounced during extended driving periods when loss of vigilance is highest (26-28).

\section{Daytime performances in sleep deprivation and (primary) insomnia}

Sleep deprivation exerts deleterious daytime effects on most of the cognitive domains (29-34). Among them, sustained attention or vigilance appears to be particularly sensitive (34-39). It can therefore be expected that driving skills highly-dependent on sustained attention abilities of drivers will be sensitive to the effects of sleep loss. A task of particular interest is prolonged highway driving, as 
optimal performance relies heavily on the availability of high levels of vigilance or sustained attention (39-47).

A recent model of attention argues that highly automated tasks such as lane keeping during highway driving are more severely affected by sleep deprivation than complex driving tasks such as overtaking, distance keeping or decision making that require top-down cognitive control (48). In order to overcome the deleterious effects of sleep deprivation on monotonous highway driving, more top-down control is thus needed to achieve normal performance. This is supported by experimental studies that consistently showed that sleep deprivation decreases road tracking control during prolonged highway driving (49-54). In contrast, operational tasks that are more demanding, such as orienting or executive attention tasks, are less affected, probably due to the reduced need for internally motivated top-down control or to the recruitment of additional mental operations (see (34)).

The impact of sleep deprivation on task performance can sometimes be minimized by increasing effort and motivation to perform a task optimally (see $(55,56)$ for reviews). However, such compensatory strategies usually fail during prolonged task performance $(55,57,58)$ regardless of whether the task demands are low or high. This effect is classically described as the Time on Task (ToT) effect which induces a progressive decline in performance and in vigilance (59). In the sleep deprivation condition, it is classically reported that sleepiness exacerbates these ToT effects (59-62).

Most of the studies which investigated driving performance after sleep deprivation were conducted in simulator settings $(49,50,54,63)$. The studies conducted in real driving conditions have compared nighttime and daytime driving without evaluating the direct effect of a night of sleep deprivation compared to a normal night of sleep on driving performance and EEG $(53,64,65)$. To our knowledge, no study on the additional effects of ToT and sleep deprivation and their EEG correlates has been conducted in real driving conditions.

Deficits in daytime performance have repeatedly been shown following sleep deprivation $(29,30,32-34,66)$. Yet, studies addressing daytime performance in PI appear inconclusive $(67,68)$. Nevertheless, a recent metaanalysis has drawn the general conclusion that PI patients display deficits in 
orientation and working memory (67). In addition, it has been shown that insomnia patients perform poorly in tasks of sustained attention that require a response choice (69-71). Overall, executive functioning of PI patients appears under-investigated. Studies using classic neuropsychological tests (e.g. trail making test B, verbal fluency, Wisconsin test, Stroop test) $(69,72-76)$ have often reported a general lack of cognitive dysfunction in PI patients (73-76). These findings contrast with results from recent neuroimaging studies that reported grey matter deficits (72) and functional hypoactivation (77) in the prefrontal cortex of patients with PI compared to control. The prefrontal cortex is a cortical area known to be involved in executive processes (78). The discrepancy between cognitive and neuroimaging results thus raises the question of the sensitivity of neuropsychological tests used to assess executive function in PI.

\section{Physiopathology of primary insomnia}

Current models of PI assume that patients are hyperaroused or unable to dearouse. This has been partly demonstrated by elevated levels of the beta power spectrum in sleep EEG in PI patients compared to good sleepers (79). In addition, these elevated levels of EEG beta power during sleep might explain why PI patients experience sleep difficulties and complain of daytime impairment $(80,81)$. Only two studies have assessed waking EEG in PI patients during daytime hours. Wolynczyk and Szelenberger (82) reported increased beta power in Multiple Sleep Latency tests in PI patients compared to good sleepers. In contrast, Wu and colleagues (83) did not observe significant differences between PI patients and normal sleepers in 5-minute resting EEG recordings with eyes open and eyes closed. So far, no study has compared waking EEG during task performance in PI patients and normal sleepers.

A few studies have investigated differences in sleep spectra between PI patients and good sleepers during REM and NREM sleep (see (79) for a review). Patients generally display increased beta activity during NREM sleep compared to good sleepers. Krystal and colleagues (84) also showed that PI patients displayed higher levels of relative alpha and sigma power compared to good sleepers during NREM sleep. These findings were partly replicated by Spiegelhader and colleagues (85), who reported increased absolute sigma power during stage 2 in 
PI patients compared to good sleepers. In contrast, a study from Merica and colleagues (86) reported lower absolute power in slower frequency bands (i.e. delta, theta, alpha and sigma) in PI patients compared to good sleepers.

Contrary to NREM findings, spectral EEG investigations during REM sleep have generally been inconsistent. Significant differences in the beta power spectrum between PI patients and good sleepers were found in two studies $(86,87)$, whereas no group differences were found in two other studies $(84,85)$. PI patients also displayed decreased absolute delta and theta power and increased alpha and sigma power compared to good sleepers (86). In addition, previous studies did not consistently investigate power spectral analysis during the whole sleep period and in all EEG bands, making it difficult to determine a general pathophysiology pattern of PI during sleep. More importantly, in light of previous findings from neuroimaging studies during the waking state $(72,77,88,89)$ and the importance of the prefrontal cortex in mediating sleep physiology $(90,91)$, no study has investigated whether specific dysfunction in prefrontal functions compared to other cortical areas may occur in PI during the entire sleep period. Only the study of Corsi-Cabrera and colleagues investigated EEG spectral analysis during the wake-sleep transition period (WSTP) and revealed increased frontal beta power compared to good sleepers during wake and during sleep stage 1 . Authors suggested a frontal deactivation dysfunction in PI during the WSTP (92).

Previous neuroimaging studies have been conducted to better understand the pathophysiology of PI and have led to various results (see (93) for a review). A finding that seems consistent is alteration in brain activity and morphology in the prefrontal cortex during the waking state in PI $(72,77,88,89)$. In addition, fMRI and spectroscopic investigations reported altered connectivity in the amygdala (94) and increased ACC volumes in PI (95), respectively. In contrast, reduced hippocampus volumes have been found in the first morphometric studies conducted in PI $(96,97)$; however, these findings were not corroborated by recent investigational results $(72,95)$.

Thus, the pathophysiology of PI is still unclear during both the waking state and the sleep period, and needs to be further investigated. 


\section{Methods}

In this dissertation, we aimed to identify the daytime performance deficits and spectral EEG modifications associated with sleep deprivation and insomnia.

\section{Measures of daytime performances}

In the current dissertation, we employed a number of tasks to measure daytime performances after sleep deprivation and in PI patients. These tasks included the Psychomotor Vigilance Task (PVT) $(98,99)$, the Attentional Network Task (ANT) (100), and prolonged highway driving tasks in both simulated and real conditions.

The PVT task is a short (about 10 minutes) laboratory measure of vigilance (98) commonly used to investigate the effects of sleep deprivation and sleepiness $(36,66,99)$. The Attentional Networks Test (ANT) is a choice reaction time task that lasts about 20 minutes. The Psychomotor Vigilance Test $(98,99)$ aimed to quantify the psychomotor vigilance of participants (Chapter 3), and the ANT was used to evaluate the integrity of the three attentional networks according to the model of Posner $(100,101)$ (Chapter2). In the current dissertation, we considered attentional processes according to the model of Posner, which states the existence of three networks that represent the alerting, orienting and executive control components of attention $(101,102)$. Alerting is defined as achieving and maintaining an alert state. Orienting consists of selecting information from sensory input. Executive control is defined as resolving conflict by choosing responses. According to psychologists and cognitive neuroscientists, vigilance, which is a key cognitive function of monotonous driving, refers to the ability to sustain attention during a task for a period time $(103,104)$. It also often refers specifically to a vigilance decrement which is the decline in attention-requiring performance over an extended period of time (105). These networks display distinct, but sometimes overlapping brain mechanisms $(106,107)$. The fMRI investigation conducted by Fan and colleagues (106) revealed the most distinct cortical and subcortical anatomical networks with the alerting contrast showing thalamic and anterior and posterior cortical activations; the orienting contrast showed parietal and frontal activations and the executive contrast revealing mainly anterior cingulate activations $(106,107)$. This study also revealed common 
activations in both the thalamus and the left fusiform gyrus for alerting and conflict contrasts, suggesting overlapping in anatomical networks.

Driving performance was evaluated by quantifying the Standard Deviation of Lateral Position (SDLP) (108). SDLP is a measure of road tracking control and sustained attention during prolonged highway driving, and can be assessed in simulated driving as well as during on-the-road driving. In addition, SDLP is one of the most sensitive driving measures for assessing driver fatigue and impairment. In Caen (Chapter 3), we used a FAROS simulator that consists of a front car cabin linked to an interactive display broadcast on one screen. The task consisted of one hour of driving in monotonous conditions (no traffic, repetitive landscape). In Maastricht (Chapter 4 and 5), the road tracking test (108) was used. In this test, participants operated a specially equipped vehicle for approximately $1 \mathrm{~h}$ over a 100-km (61 miles) primary highway circuit accompanied by a licensed driving instructor having access to dual controls (brakes and accelerator). In both driving settings, participants were instructed to drive as straight as possible within the right traffic lane while maintaining a constant speed.

\section{Using EEG to measure attention and vigilance fluctuations during driving and fine-grained sleep modifications}

One of the classic methods used to investigate fluctuations in vigilance and attention during task performance is spectral EEG (103,109-111). Changes in waking spectral EEG during vigilance and sleepiness fluctuations in normal conditions are well understood $(112,113)$, and several studies have reported the feasibility of measuring EEG fluctuations (i.e. variations in EEG power spectrum) in sustained attention tasks (114), specifically during driving experiments (see (103), for a review).

Most studies that have investigated EEG during driving have been conducted under simulated driving conditions. These studies generally reported slowing of EEG with ToT reflected by decreases in beta, and increases in alpha and theta power to coincide with reductions in vigilance during prolonged driving $(63,115,116)$. The alpha EEG band is related to cortical excitability (117-120). Therefore, a reduced alpha power spectrum could represent an (hyper)aroused state such as that which was found to characterize insomnia patients (81). 
Consequently, increments in the alpha EEG band are believed to represent sleepiness $(121,122)$. In addition, the alpha EEG band is related to attentional processing $(118,120,123,124)$ and is especially attenuated in the case of maximal attention (125-128) and increased cognitive demand $(129,130)$. Fluctuations in the beta EEG power spectrum have been associated with changes in vigilance $(131,132)$. Several cognitive investigations have reported that the beta power spectrum increases as a function of ToT $(115,133)$. It has been suggested that beta increments primarily reflect a compensatory effort to maintain high levels of performance as vigilance decreases (134). In contrast, a driving study conducted in conditions of normal sleep reported beta power spectrum and attentional level decreases over time (135). Therefore, interpretation of beta power during vigilance tasks is not completely clear even though this parameter is often used to discriminate performance decrements during cognitive and driving tasks $(131,135)$. The theta EEG band is related to sleepiness and fatigue $(115,136)$ and commonly increases as a function of ToT during sustained attention tasks $(111,115,137)$. The two measures that most often correlate with worse performance are increasing theta and decreasing beta power spectra (125).

Spectral EEG analysis is sometimes considered to be a more promising measure of sleep processes in PI than the classically polysomnography derived indices according to the Rechtschaffen and Kales criteria (79). This method allows the decomposition of the raw EEG into an infinite number of pure sinusoidal components, each of a different frequency, which when added together again yield the original signal $(138,139)$. The Fast Fourier transformation (FFT; $(140))$ is a widely applied method used to obtain the EEG spectrum in each frequency band. Spectral power analysis quantifies the amplitude of each constituent wave (or its square: power) and is able to separate slower and faster parts of the EEG. In the current dissertation, we computed relative power spectra in the four major frequency bands: delta $(1.5-4 \mathrm{~Hz})$; theta $(4-7.5 \mathrm{~Hz})$; alpha (separated in alpha1, alpha 2 and alpha 3 and aggregated) $(7.5-12.5 \mathrm{~Hz})$; sigma $(12.5-14 \mathrm{~Hz})$; beta (separated in beta1, beta 2 and beta 3 and aggregated) $(14-30 \mathrm{~Hz})$. All night power averages were obtained for stage 2, stage $3-4$ and REM sleep separately. In addition, we compared the band activity within the prefrontal electrodes to the 
average of the other main electrodes (central, parietal, occipital) in order to highlight specific EEG sleep patterns in the prefrontal cortex in PI patients.

\section{Aims}

The aims of this dissertation were:

- Evaluate daytime performances (as a function of ToT) in PI patients and in healthy volunteers after sleep deprivation,

- Investigate the pathophysiology of PI by using EEG to determine brain activity modifications both during both the daytime and the sleep periods.

\section{Outline of this dissertation}

In Chapter 2, attentional performance of PI patients and good sleepers was compared. The Attentional Network Task (ANT) was employed to assess performance differences in three main attentional netwoks according to Posner (i.e alerting, orienting and executive control). In this study we aimed to better characterize cognitive deficits of PI patients in order to further understand their cognitive complaints.

Chapters 3 and 4 describe two experiments conducted to evaluate the driving performance of PI patients and good sleepers during a prolonged highway driving task in simulated and real driving conditions, respectively. The major driving parameter was the SDLP, which is a measure of road tracking control and sustained attention during driving. In addition, EEG power spectra were assessed during an on-the-road driving test (Chapter 4) to obtain psychophysiological indices of vigilance and sleepiness during driving. The major aims of these studies were to assess daytime deficits in driving quality of patients with PI and to assess the relationship between background EEG and driving performance changes.

In Chapter 5, on-the-road driving performance and background EEG were assessed in healthy volunteers after a normal night of sleep and after a night of sleep deprivation. SDLP was used as the major parameter of vigilance during prolonged highway driving. The study aimed to determine the influence of sleep deprivation on driving performance as a function of the time on driving task. This 
chapter also discusses the cumulative effects of sleep deprivation and ToT in conditions of real driving.

Chapter 6 presents a study on spectral EEG during sleep in PI patients and good sleepers. The analyses focus on spectral EEG in the prefrontal cortex that has been considered to play a major role in the pathophysiology of PI. The major aim of this study was to identify modifications in sleep spectra in the prefrontal cortex compared to other cortical areas in PI patients by using EEG.

In Chapter 7, each chapter is discussed in the general context of PI and sleep deprivation in order to draw general conclusions that aimed at achieving the two main aims of this dissertation. First, the daytime performances associated with PI and sleep deprivation are discussed with a particular focus on ToT effects on driving performances and EEG fluctuations. Second, by discussing our results, new hypotheses are suggested and preliminary answers are given about the pathophysiology of PI. Finally, the relationship between EEG and driving performance fluctuations are discussed and other measures to predict driving performance are proposed. Finally, new prospective future research is described in relation to the findings in the current dissertation. 


\section{Attention and vigilance deficits in primary insomnia}





\section{Chapter 2 \\ Attentional networks and vigilance in primary insomnia}

Accepted as:

Perrier, J., Chavoix C., \& Bocca, M.-L. Functioning of the three attentional networks and vigilance in primary insomnia. Sleep Medicine. 


\begin{abstract}
Results from cognitive measures in primary insomnia (PI) patients do not fit with the difficulties in performing daily living tasks of which these patients complain. Lack of sensitivity of the tests and lack of data concerning some cognitive functions may explain this discordance. The aim of the present investigation was to better characterize cognitive deficits of PI patients in order to further understand their cognitive complaints. We looked at attentional and executive function because of their high involvement in daily living tasks. We only included untreated PI patients since sleep medication could be a confounding factor when assessing cognition. Twenty-one PI patients and sixteen good sleepers completed the Attentional Network Test (ANT). No difference between groups was found for each of the three attentional network performances (i.e. alerting, orienting, and executive function). However, in contrast to controls, PI patients performed more slowly in the incongruent flanker condition (i.e conflict situation) than in the congruent flanker condition, reflecting a conflict resolution deficit. This impairment may be linked to the prefrontal alterations reported in neuroimaging studies in these patients. We found a longer overall reaction time in PI patients than in good sleepers, reflecting impaired vigilance likely due to the high cognitive load of the ANT. Together with the executive dysfunction, this result would serve to explain the complaints of PI patients for whom performing daily living tasks is often long-lasting and demanding.
\end{abstract}




\section{Introduction}

Among the various subtypes of insomnia, primary insomnia (PI) affects between 2 and $4 \%$ of the adults in the general population (141). Daytime function and subjective complaints have been considered to be core features in the diagnosis of PI. Despite these complaints, previous studies have failed to demonstrate objective evidence of a cognitive dysfunction in PI (67). Nonetheless, frequent deficits in tasks of "orientation of attention" and working memory have been highlighted (67). Among the other cognitive domains, executive function remains under-investigated (67), and the rare studies in this field have shown inconsistent results. The lack of executive dysfunction observed by some authors (73-76) contrasts with the results from neuroimaging studies in PI which revealed structural and functional alterations in the prefrontal cortex $(72,77,89)$, a cortical area known to be involved in executive functions (78). This discrepancy between cognitive and neuroimaging results raises the question of the sensitivity of the classic tests used to assess executive function in PI (e.g. Trail making test B, verbal fluency, Wisconsin test, Stroop test) (72-77).

Performance of vigilance and sustained attention, and choice reaction time tasks are consistently impaired in PI (69-71), at variance with simple reaction time tasks $(69,70,75,142,143)$. A vigilance decrement in PI was also found in a recent monotonous driving investigation (144). Thus, it appears that mainly tasks with a high cognitive load are impaired in PI.

Altogether, these data suggest that choice reaction tasks with a high cognitive load should be most appropriate for detecting cognitive deficits in PI. The Attentional Network Test (ANT) $(100,101,107)$ seems a good option for addressing this issue. It is a suitable task to use for assessing, within a single task, executive control, orientating, and alerting. The ANT, which combines a cue detection task (101) and a flanker paradigm (145), is a complex choice reaction time test of about $25 \mathrm{~min}$ in duration in which participants have to report the orientation of a central arrow presented with or without distracters. In addition to the usefulness of the ANT as a powerful cognitive tool, the neural basis of the three networks has been well defined using neuroimaging investigations $(100,106,107)$. 
In this study, we aimed to better characterize cognitive deficits in PI patients in order to further understand their cognitive complaints. We looked at attentional and executive function because of their high involvement in daily living tasks, and because of the lack of data on executive function in PI. In addition, we included only untreated PI patients because sleep medication, particularly benzodiazepines, could be a confounding factor when assessing cognition (146). Thus, we compared orienting, alerting, and executive effects between a group of untreated PI patients and a group of good sleepers using the ANT. We hypothesized that PI patients would have poorer performance in executive control and orienting, and longer global reaction time than good sleepers.

\section{Methods}

\subsection{Participants}

Twenty-one participants suffering from primary insomnia and without use of sleep-promoting agents ( 9 men and 12 women; mean age $=48 \pm 16$ years; age range $=24-77$ years $)$ and sixteen good sleepers $(6$ men and 10 women; mean age $=48 \pm 14$ years; age range $=23-64$ years) were included in this study. They were recruited via announcements posted in public transports, medical centers, and newspapers. Following a telephone interview, participants from both groups were carefully examined by a sleep clinician to ascertain the primary insomnia diagnosis according to the DSM-IV for patients, and good sleep conditions for good sleepers.

Participants included in the good sleepers group had to be self-defined as good sleepers, which was confirmed by polysomnography. Participants included in the insomnia group had to meet the following inclusion criteria according to DSM-IV primary insomnia criteria: (a) presence of a subjective complaint of insomnia for at least six months and with a minimum of three nights per week, defined as (i) difficulty initiating sleep (sleep onset latency (SOL) $>30 \mathrm{~min}$ ) and/or maintaining sleep (time awake after sleep onset $>30 \mathrm{~min}$ ), (ii) early awakening (waking up earlier than the desired wake time), or (iii) insufficient amount of sleep $(<6.5 \mathrm{~h}$ of sleep); (b) insomnia or its perceived consequences causing marked distress or significant impairment of occupational or social function (e.g. 
problems of concentration); and (c) presence of a subjective complaint of at least one negative daytime consequence attributed to insomnia (e.g. fatigue, mood disturbances).

The exclusion criteria for both groups were: (a) current medical or neurological disorder that could compromise sleep; (b) serious psychopathology that could induce insomnia; (c) consumption of psychotropics or other medications known to alter or induce sleep as assessed with blood and urine screening the day of the experiment; (d) poor hygiene or habitual abnormal sleep patterns (e.g. night or shift work); and (e) other sleep disorders (assessed by polysomnography) such as sleep apnea (apnea-hypopnea index $>10$ ), or periodic limb movements during sleep (myoclonic index with arousal $>10$ ). Participants from both groups were also excluded if they: (a) had current or past dependence on alcohol, opiates, benzodiazepines, or any illicit drugs; (b) smoked more than 5 cigarettes per day; (c) drank more than 28 units of alcohol per week; (d) consumed more than $150 \mathrm{mg}$ of caffeine per day; (e) did not have normal or corrected to normal vision (visual acuity lower than $7 / 10$ ). This protocol was granted ethical approval by the Caen Northwest III ethics committee and by the Health Ministry (number DGS 2005/0388). Each participant provided written informed consent in accordance with the requirements of the committee.

\subsection{General procedure}

The data for this paper were recorded as part of a study that assessed driving performance and sleep of PI patients and good sleepers (144). For the present paper, ANT data were analyzed and compared between the two groups.

Participants underwent a night of polysomnography the day before the test day and were brought to the laboratory the next morning for the testing day. Urine and blood samples were taken to confirm their compliance with prohibitions against prior use of oral medication. The ANT was given at a similar time in both group (after the lunch break) because performance may vary with time of day (147). 


\section{The Attention Network Test (ANT)}

Participants were asked to complete the ANT in front of a computer and responses were collected through the keyboard of the computer. For a detailed description of both stimuli and procedure see the study of Fan and colleagues (100). Stimuli consisted of a row of five horizontal black lines with arrowheads pointing leftward or rightward presented on a grey background. The target was a left- or rightpointed arrow located on the center of the screen, which was flanked by two arrows (flankers) on each side pointing in the same or in the opposite direction from the target (congruent trials and incongruent trials, respectively), or that were replaced by lines (neutral trials). A single arrow or line subtended $0.558^{\circ}$ of visual angle. The participants' task was to identify the direction of the centrally presented arrow on the screen by pressing buttons as fast as possible with the index finger of the right hand to indicate the direction of the arrow (the right button for the right direction and the left one for the left direction). A cue (a star) was presented before the presentation of the target. The cues could be: i) absent (no trials); or ii) presented at the center of the screen (center cue trials); iii) presented above or below the target place (spatial cue trials); or iv) presented simultaneously above and below the target (double cue trials). Therefore, three Flanker conditions (neutral, congruent, or incongruent) and four Cue conditions (no cue, center cue, spatial cue, and double cue) were possible. The cues and flankers were presented $1.06^{\circ}$ above or below the fixation point.

Each trial began with the presentation of a fixation cross in the middle of the computer screen. Subjects were instructed to keep their eyes fixed on the cross during the entire duration of the test. Then, at variable intervals (ranging from 400 to 1600 milliseconds), a cue was shown for 100 milliseconds. Four hundred milliseconds after the offset of the cue, a flanker display appeared, and remained on until the participant's response, or for 1700 milliseconds if no response was given. The inter-stimulus interval was of 3500 milliseconds. After a 24-trial practice block, participants performed three experimental blocks of 96 trials each.

\subsection{Statistical analysis}

Characteristics of participants, the $t$-test, and Welch test were used if the normality hypothesis could not be rejected by the Shapiro-Wilk test. For non- 
Gaussian samples, preference was given to the Wilcoxon-Mann-Whitney test (See Table 1). Global performance of the participants (i.e. mean reaction time (RT)) was analyzed using a 3-ways repeated measures ANOVA with Group (Insomnia patients, Good sleepers) as a between-participant factor, and Cues (Spatial, Central, Double, No-Cue) and Flankers (Congruent, Incongruent, Neutral) as within participant repeated factors. In addition, alerting, orientating, and executive control performances were evaluated by measuring the influence of cue and flanker conditions on RT relative to a referent condition according to Fan and colleagues $(100,106)$, as follows: RT no cue minus $\mathrm{RT}$ center cue for alerting, RT center cue minus RT spatial cue for orienting, and RT incongruent flankers minus RT congruent flankers for executive function. Then, intergroup comparison was performed for each attentional component with one-way ANOVA. Post hoc analyses were performed with the Tukey test. The size effects were calculated with $\mathrm{Eta}^{2}$. The level of statistical significance was set at $p<0.05$.

Statistical analyses were carried out using Statistica ${ }^{\circledR}$ software version 10.

\section{Results}

\subsection{Participant characteristics}

The characteristics of participants are given in Table 1. Participants differed in their sleep quality (i.e. ISI and PSQI), but not in their circadian characteristics, daytime sleepiness (i.e. Epworth), nor in age. Due to technical problem, the ANT data from two participants could not be collected in the PI group. 
Table 1. Characteristics of the insomnia patient and good sleeper groups, and statistical differences between groups.

\begin{tabular}{cccc}
\hline Characteristics & $\begin{array}{c}\text { Insomnia patients } \\
\text { Mean (SD) }\end{array}$ & $\begin{array}{c}\text { Good sleepers } \\
\text { Mean (SD) }\end{array}$ & $\boldsymbol{p}$ values \\
\hline ISI & $16.00(4.96)$ & $2.55(1.64)$ & $<\mathbf{0 . 0 0 0 1}$ \\
PSQI & $10(2.72)$ & $4(2.07)$ & $<\mathbf{0 . 0 0 0 1}$ \\
Chronotype & $57.81(9.13)$ & $60.33(8.7)$ & 0.42 \\
ESS & $6.9(4.55)$ & $6.2(3.88)$ & 0.65 \\
Age & $48.71(16.18)$ & $48.31(14.21)$ & 0.94 \\
\hline
\end{tabular}

ISI, Index of Insomnia severity (score and sleep difficulties increase proportionately; score $>7$ is considered to be an index of insomnia); PSQI, Pittsburgh Sleep Quality (score and sleep difficulties increase proportionately); Chronotype (according to Horne and Ostberg (148): 70-86=definitively morning type, 5969=moderately morning type, 42-58=neither type, 31-41=moderately evening type, 13-30=definitively evening type) ; ESS, Epworth Sleepiness Scale, daytime sleepiness questionnaire (score and sleepiness increase proportionately).

\subsection{Attentional Network Test Performance}

Separate one-way ANOVA performed to evaluate alerting, orienting, or executive control effects did not revealed any group difference (See Table 2). Detailed performance in the PI patient and good sleeper groups for each Cue and Flanker condition in the ANT are shown in Figure 1.

Table 2. Mean reaction times (RT) and standard deviations (SD) for the alerting, orienting, and executive networks in the insomnia patients and in good sleepers, together with the statistical results.

\begin{tabular}{cccccc}
\hline Attentional networks & $\begin{array}{c}\text { Insomnia patients } \\
\text { Mean (SD) }\end{array}$ & $\begin{array}{c}\text { Good sleepers } \\
\text { Mean (SD) }\end{array}$ & df & F & p value \\
\hline Alerting (ms) & $37.64(24.14)$ & $39.03(28.80)$ & 1 & 0.023 & 0.88 \\
Orienting (ms) & $42.11(35.45)$ & $36.66(26.12)$ & 1 & 0.26 & 0.62 \\
Executive control (ms) & $119.94(43.30)$ & $105.56(34.04)$ & 1 & 1.138 & 0.29 \\
\hline
\end{tabular}

df: degrees of freedom 


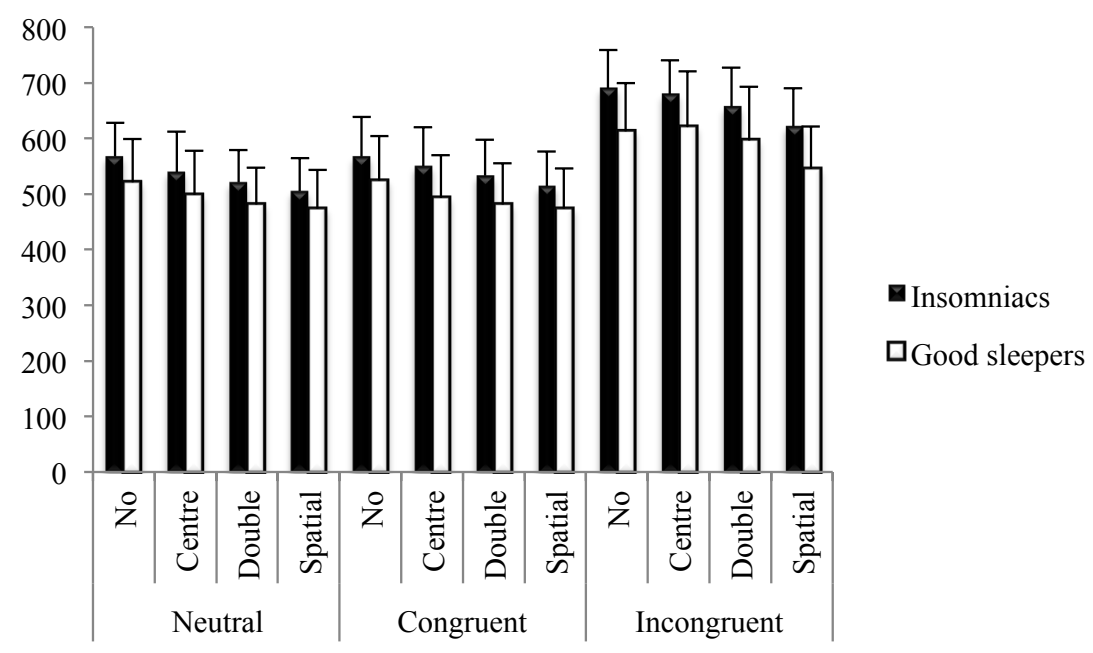

Figure 1. Reaction time (in ms) for each Flanker and each Cue condition in patients with primary insomnia and in good sleepers. Error bars represent standard deviations.

Statistical results with the 3-way repeated measures ANOVA (2 Groups, 4 Cues, and 3 Flankers) performed on the mean RT for the ANT are summarized in Table 3. They showed a main effect of Group, Cue, and Flanker as well as significant Flanker by Group and Cue by Flanker interactions. The remaining interactions (Cue by Group and Cue by Flanker by Group) did not reach significance. 
Table 3. Statistical results of the 3-way repeated measures ANOVA on the reaction time for the Attentional Networks Test (ANT).

\begin{tabular}{lcccc}
\hline Effects & Df & $\mathbf{F}$ & $\boldsymbol{p}$ value & Partial eta $^{2}$ \\
\hline Group & 1 & 5.24 & $\mathbf{0 . 0 2 9}$ & 0.14 \\
Cue & 3 & 164.36 & $<\mathbf{0 . 0 0 0 1}$ & 0.84 \\
Flanker & 2 & 15.43 & $<\mathbf{0 . 0 0 0 1}$ & 0.33 \\
Cue $^{*}$ Group & 3 & 2.22 & 0.090 & 0.064 \\
Flanker $^{*}$ Group & 2 & 3.27 & $\mathbf{0 . 0 4 4}$ & 0.092 \\
Cue*Flanker $^{*}$ & 6 & 118.97 & $<\mathbf{0 . 0 0 0 1}$ & 0.79 \\
Cue*Flanker $^{*}$ Group & 6 & 0.94 & 0.47 & 0.029
\end{tabular}

Cohen (149) provided the following guidelines for interpreting the eta squared value: $0.01-0.059=$ small effect size, 0.06-0.139=medium effect size, $>0.14=$ large effect size. Df, degrees of freedom.

As shown in Figure 2, overall RT was longer in PI patients $(575.26 \pm 90.58 \mathrm{~ms})$ than in good sleepers $(519.30 \pm 94.58 \mathrm{~ms})$. For the Flanker* Group interaction, post hoc analyses revealed that RT for incongruent flankers $(662.20 \pm 127.33 \mathrm{~ms})$ was significantly longer than that for neutral $(533.20 \pm 65.85 \mathrm{~ms})$ and congruent flankers $(541.0 \pm 68.64 \mathrm{~ms})$ in the insomnia group $(p<0.001$, for both) whereas no significant difference in RT was found between the different Flanker conditions in the good sleepers group (see Figure $3)$. As expected, post hoc analyses showed that RT for each Cue condition was significantly different from one other ( $p<0.05$ for each comparison). For the Cue by Flanker interaction, it was due to a longer RT for the center Cue condition in the incongruent Flanker condition $(652.10 \pm 86.08 \mathrm{~ms})$ than in the Spatial Cue $(585.80 \pm 80.94 \mathrm{~ms})$, no Cue $(654 \pm 86.49 \mathrm{~ms})$, and double Cue conditions $(628.30 \pm 87.95 \mathrm{~ms})(p<0.05$ for each comparison) across both groups. Our significant comparisons correspond to effect sizes above 0.14 , a value generally considered as reflecting large effect sizes (149). 

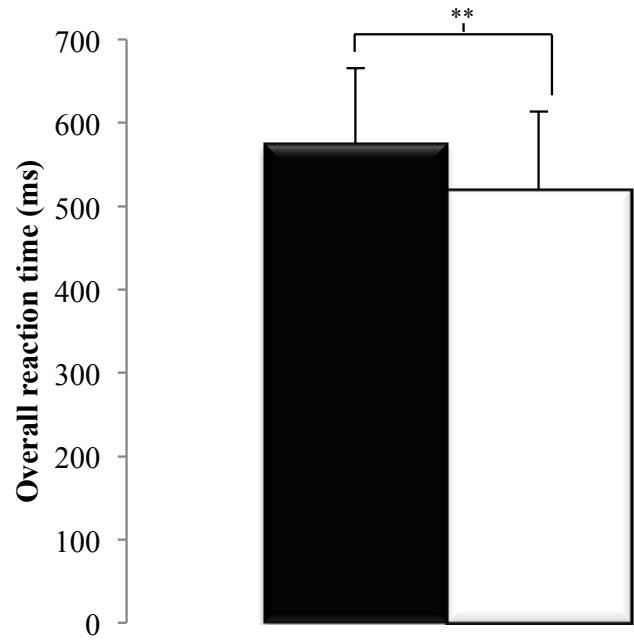

EInsomniacs

$\square$ Good Sleepers

Figure 2. Overall reaction time (i.e. global reaction time for all Flanker and Cue conditions) for the Attentional Networks Test in patients with primary insomnia patients and in good sleepers. Vertical bars are standard errors for each group; ${ }^{* *} p<0.05$.

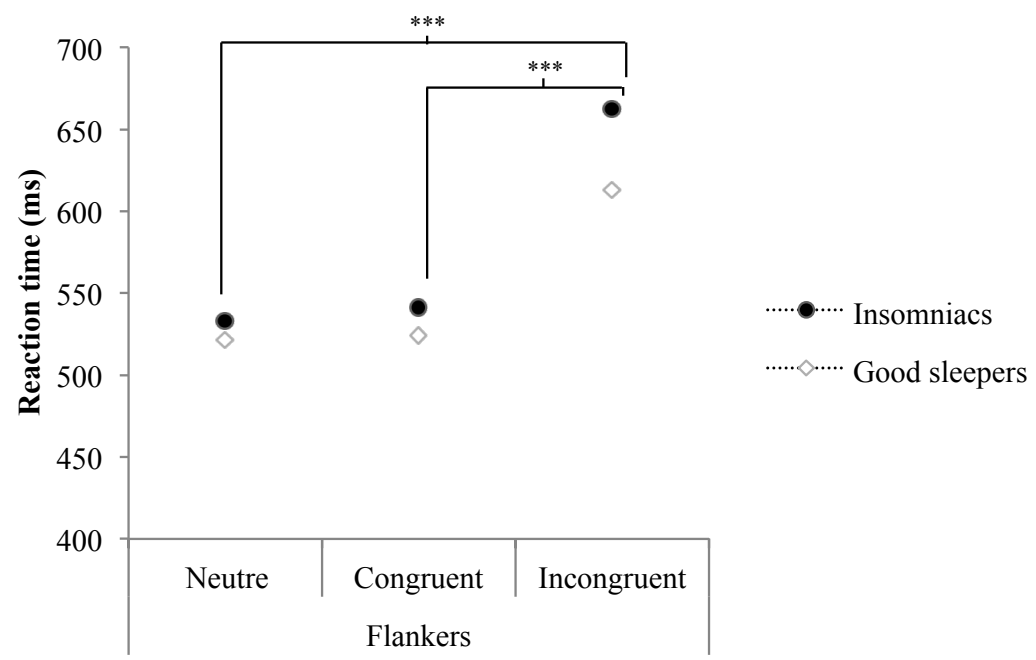

Figure 3. Details of the reaction times (in ms) for each Flanker condition in patients with primary insomnia and in good sleepers. $p<0.001^{* * *}$. Note that significant differences between flanker conditions involve only the insomnia patients group. Error bars are not displayed since each participant was his own control in the repeated measures ANOVA. 


\section{Discussion}

Our findings show that PI patients have alerting, orienting, and executive control performances that are similar to good sleepers. However, an increased RT for the incongruent flanker condition compared to neutral and congruent flanker conditions was found in the group of PI patients only. Moreover, the overall RT was significantly longer in PI patients than in good sleepers.

The lack of difference in performance between PI patients and good sleepers for alerting, orienting, and executive attention suggests the integrity of these three attentional networks in PI. However, the increased RT for the incongruent flankers compared to the other flanker conditions found in the PI group only argues in favor of slower reaction in PI patients when faced with a conflict situation. A methodological issue may explain these discrepant findings regarding executive control in PI. The three-way ANOVA that reveals a "Flanker by Group" interaction includes the 3 Flanker conditions (neutral, congruent, incongruent), and thus takes into account all aspects of the ANT task. By contrast, the one-way ANOVA performed on each attentional network performance compares two types of flankers only (congruent vs incongruent). Thus, by allowing a more in-depth analysis, the three-way ANOVA demonstrates impaired executive function in PI patients.

The conflict process is one of the numerous processes included in the term executive function. The executive function is defined as complex cognitive processing that requires the coordination of several subprocesses to achieve a particular goal (150). Executive control includes a large range of abilities such as initiating, planning, setting goals, solving problems, regulating emotions, and monitoring behavior $(102,150,151)$. Alterations have been found only for some aspects of executive function in PI. Impairment has been reported in problem solving but not in planning, reasoning, mental flexibility, or multitasking (67). The present study reveals that the conflict process is also impaired in PI. This result agrees with Liu's study in which the ANT was also used in PI patients (152). In their study, as PI patients on medication were included, the impaired executive control performance observed could have been the result of the insomnia disorder per se or of the deleterious effects of medication on cognition (146). The results 
of the present study indicate that PI per se leads to executive dysfunction. In addition, this study underlines the sensitivity of the ANT in revealing a conflict resolution deficit in PI. Indeed, Orff's study (74) using the Stroop task (another conflict task) did not show any impairment in PI patients.

Neuroimaging studies have shown prefrontal modifications in PI, a key cortical area for executive function (78). For instance, atrophy and hypoactivation during a fluency task have been reported in the dorsolateral prefrontal cortex (DLPC) of PI patients $(72,77)$. fMRI and spectroscopic investigations have also revealed increased volume in the anterior cingulate cortex (ACC) (95), a prefrontal area known to be part of the anatomical network common to various conflict tasks (e.g. Flanker task, color and spatial Stroop tasks) $(107,153)$. It has been reported that the Flanker task, which is part of the ANT, produces more interference and leads to greater activation in the ACC than the Stroop task (153). Thus, we hypothesize that the ANT, by more strongly recruiting the ACC than the Stroop task, was more appropriate than the Stroop task for highlighting conflict process impairment in PI. Therefore, a combined investigation using ANT and fMRI would be useful for gaining better knowledge about the recruitment of various cortical areas during the whole conflict resolution process in PI.

The normal performance of alerting and orienting networks in PI is in line with a previous report in which the ANT was also used (152). However, impairment in the orientation component of attention has been reported in PI using other tasks (154-158). These discrepant findings could be explained by the sleep-related stimuli used to assess the attentional bias in all of these studies that found impaired orientation. Because sleep is anxiety-producing in PI patients $(80,159)$, such stimuli may have disturbed the tests. Further investigations that aim to compare orienting performance in PI patients using different stimuli, namely sleep-related vs neutral stimuli, are needed to confirm that sleep-related stimuli could induce a bias in the attentional assessment of those suffering from insomnia. Other highly demanding tasks eliciting alerting and orienting functions should also be further investigated in PI. For example, oculomotor paradigms have proved to be very useful in assessing cognitive functions, particularly spatial attention and executive function, as shown after sleep deprivation and drugs intake $(147,160,161)$. 
Finally, our result revealed a longer overall reaction time in PI patients compared to good sleepers. This result likely reflects impaired vigilance, as interpreted in light of similar findings using ANT in Attention Deficit Hyperactivity Disorder (162-164) and after sleep deprivation (165). Such vigilance decrements in PI during a long (almost 25 minutes) and high-cognitive demanding task (choice reaction time task) are consistent with previous reports. Previous investigations have revealed a vigilance decrement in PI patients by using challenging tasks such as a choice reaction time task (71), a Continuous Performance Test (70), or a complex vigilance task (69), a simple but long-lasting vigilance task (40 minutes) (142), and a monotonous one-hour driving task (144). By contrast, vigilance is not impaired in PI using tasks that are both simple and short-lasting such as the PVT $(74,75,143,166,167)$.

Using a long-lasting and highly demanding attentional task, this study contributes by making the cognitive complaints of PI patients more objective. Complaints of daytime cognitive difficulties are part of the PI diagnosis. PI patients have complaints of memory problems, difficulty with concentration and decision-making, and work related errors $(168,169)$. These complaints are related to tasks that engage various cognitive and attentional functions. Numerous laboratory studies have failed to detect cognitive impairments in PI patients (see (67), for meta-analysis and (68), for review), probably because the tasks used did not engage some key characteristics of daily living tasks, which seem to be accurately brought together in the ANT.

In conclusion, the present study reveals for the first time that untreated PI have a conflict resolution deficit, in agreement with the prefrontal alterations previously reported in these patients. Together with the vigilance decrement probably linked to the highly demanding situation of the task used, this result serves to highlight the difficulty for patients with PI for whom carrying out daily living tasks is often long-lasting and highly demanding. 


\section{Chapter 3 \\ Impaired driving performance associated with effect of time duration in patients with primary insomnia}

Published as:

Perrier, J., Bertran, F., Marie, S., Couque, C., Bulla, J., Denise, P., \& Bocca, M.-L. (2014). Impaired driving performance associated with effect of time duration in patients with primary insomnia. Sleep, 37(9), 1565-1573. 


\begin{abstract}
Insomnia has been associated with an increased risk of having road accidents according to epidemiological studies. However, until now, no experimental study has been perfrmed to evaluate the driving performance of primary insomnia patients. The aim of the current investigation was to evaluate driving performance and psychomotor vigilance in patients with primary insomnia. For this purpose twenty-one primary insomnia patients and sixteen good sleepers performed a onehour simulated monotonous driving task and a psychomotor vigilance task (PVT). Self-ratings of sleepiness, mood, and driving performance were also completed. Results revealed a larger standard deviation of lateral position $(p=0.023)$ and more lane crossings $(p=0.03)$ in insomnia patients than in good sleepers. Analyses of time on task effects on performance showed that the impairment in patients occurred after $20 \mathrm{~min}$ of driving, which was not the case for good sleepers. No difference between groups was found for the PVT, neither for the mean reaction time $(\mathrm{RT})(p=0.43)$ nor the number of lapses $(p=0.21)$ and the mean slowest $10 \% 1 / \mathrm{RT}(\mathrm{P}=0.81)$. Patients rated their sleepiness level higher $(p$ $=0.06)$ and their alertness level lower $(p=0.007)$ than did good sleepers $(p=$ 0.007). The self-evaluation of the driving performance was not different between groups $(p=0.15)$. These findings revealed that primary insomnia is associated with a performance decrement during a simulated monotonous driving task. We also showed that patients are able to drive safely only for a short time. It appears advisable for clinicians to warn patients about their impaired driving performance that could lead to an increased risk of driving accidents.
\end{abstract}




\section{Introduction}

One of the most prevalent sleep disorders is insomnia, which affects from $5 \%$ to $30 \%$ of the general population $(6,7,170)$. The wide range of prevalence values is mainly linked to the definition used to assess insomnia which differs across studies (5-7,171-174). The current work focuses on primary insomnia. According to the Diagnostic Statistical Manual of Mental Disorders, $4^{\text {th }}$ Edition (DSM-IV) definition, insomnia is a complaint of nonrestorative and/or insufficient sleep with various symptoms, such as difficulties in initiating and maintaining sleep and early morning awakenings associated with complaints of daytime consequences without comorbidity. Using this definition, the prevalence of primary insomnia was found to occur in $3-5 \%$ of the general population ( 7 ).

Insomnia is a public health problem, with annual direct and indirect costs estimated at $\$ 1,253$ greater than for patients without insomnia in the United States (15); these costs were estimated at $\$ 5,010$ per year in the province of Quebec, Canada (16). These costs are the result of reduced productivity, higher work absenteeism, and non-motor vehicle accidents in patients with insomnia in comparison with good sleepers $(11,13,175)$. Another consequence of insomnia is the increased risk of road accidents. Sleep disorders comprise one of the many factors suspected of increasing the risk of road accidents (20). Insomnia was associated with a 2.5- to 3-fold higher risk of serious road accidents in comparison with good sleepers, according to a French survey (176). However, no experimental studies have been performed to evaluate the effects of insomnia on driving performance. Staner and colleagues (177) showed that patients with insomnia treated with hypnotics had impaired driving performance in comparison with placebo-treated patients with insomnia. This study did not include a control group of good sleepers. Consequently, because the use of hypnotics is associated with impaired driving performance (178-180) and increased risk of driving accidents (181-183), whether insomnia without hypnotic treatment leads to impaired driving performance in comparison with good sleepers has not been established. The primary objective of the current study was therefore to evaluate the effects of untreated insomnia on driving performance. 
Driving a car requires the possession of sufficient cognitive, visual, and motor skills, and involves managing attention in order to perform various drivingand non driving-related tasks (184). Epidemiological and experimental studies have focused on some factors leading to road accidents or driving impairments. Sleepiness and fatigue are often cited (185). For example, it has been demonstrated that sleepiness is responsible for almost $15-20 \%$ of road accidents $(24,25,186-189)$. Because patients with primary insomnia have not been found to be sleepy in most studies (67), Smolensky and colleagues (20) hypothesized that the increase in car crashes may be caused by other factors, such as daytime fatigue and cognitive impairment, which are consistently reported. The secondary objective of this study was to assess the effects of untreated insomnia on a cognitive function, i.e., vigilance. For this, we used the classic test used in sleep deprivation and driving studies, the Psychomotor Vigilance Test (PVT), which is based on a simple visual reaction time (99,190-193). Assessment of subjective feelings, i.e., change of mood, alert feelings, and subjective driving performance evaluation, were assessed using the Karolinska Sleepiness Scale (KSS) (121), a Visual Analog Mood Scale (VAMS) (194), and a driving scale (195).

\section{Methods}

\subsection{Participants}

Participants were recruited via announcements posted in public transportation centers, medical centers, and newspapers. Following a telephone interview, participants from both groups were carefully interviewed by a sleep clinician to ascertain: (1) sleeping difficulties and diagnosis of DSM-IV insomnia for the insomnia patients group; and (2) good physical condition: the absence of sleep, alertness, neurological, cardiovascular, respiratory, hepatic, renal, or metabolic disorders, and absence of poor hygiene or habitual abnormal sleep patterns (e.g., night or shift work) for the good sleepers group.

Participants included in the insomnia group had to meet the following inclusion criteria according to DSM-IV primary insomnia criteria: (1) presence of a subjective complaint of insomnia, defined as difficulty initiating (sleep onset latency (SOL), >30 min) and/or maintaining sleep (time awake after sleep onset $>30 \mathrm{~min})$; (2) early awakening $(<6.5 \mathrm{~h}$ of sleep or waking up earlier than the 
desired wake time); (1) and (2) had to occur at least 3 nights per week; (3) insomnia for at least $6 \mathrm{mo}$; (4) insomnia or its perceived consequences causing marked distress or significant impairment of occupational or social function (problems of concentration); and (5) presence of a subjective complaint of at least one negative daytime consequence attributed to insomnia (e.g., fatigue, mood disturbances).

The exclusion criteria were: (1) current medical or neurological disorder that could compromise sleep; (2) serious psychopathology that could induce insomnia; (3) consumption of psychotropic or other medications known to alter or induce sleep; (4) poor hygiene or habitual abnormal sleep patterns (e.g., night or shift work); and (5) other sleep disorders (assessed by polysomnography [PSG]) such as sleep apnea (apnea-hypopnea index $>10$ ), or periodic limb movements during sleep (myoclonic index with arousal $>10$ ). All participants with insomnia and good sleepers were excluded if they: (1) had current or past dependence on alcohol, opiates, benzodiazepines, or any illicit drugs; (2) smoked more than five cigarettes per day; (3) drank more than 28 units of alcohol per week; or (4) consumed more than $150 \mathrm{mg}$ of caffeine per day. All participants had normal or corrected to normal vision (visual acuity greater than or equal to $7 / 10$ ). They had all driven regularly for at least $2 \mathrm{y}$ and drove at least $5,000 \mathrm{~km} / \mathrm{y}$.

Twenty-one participants suffering from chronic primary insomnia (9 men and 12 women; mean age $=48 \pm 16$; age range $=24-77$ y) and $16 \operatorname{good}$ sleepers ( 6 men and 10 women; mean age $=48 \pm 14$; age range $=23-64$ y) were included in this study. It was granted ethical approval by the Caen Northwest III ethics committee and by the Health Ministry (number DGS 2005/0388). Each participant provided written consent in accordance with the requirements of the committee.

\subsection{Experimental Procedure}

To avoid motion sickness, participants were familiarized with the simulator $1 \mathrm{w}$ before the experimental day. None of the participants suffered from motion sickness. The day before the tests (driving, self-assessments, and PVT), the participants arrived at the sleep unit at 20:30 for a PSG recording. Lights out was 
initiated at 22:30 and participants were awakened at 07:00 the following morning by the experimenter.

After a standardized breakfast, participants were brought to the laboratory to perform the tests. At 08:30, blood and urine samples were collected for routine laboratory drug screening for alcohol, opiates, cocaine, cannabis, amphetamines, and barbiturates.

At 09:00 (i.e, $2 \mathrm{~h}$ after waking up), participants were brought to the simulator room to complete a 1-h simulated driving test. After a short break, they completed self-assessment scales at approximately 10:15 (i.e., $3 \mathrm{~h}$ and $15 \mathrm{~min}$ after waking up) relating to their subjective sleepiness (KSS), their subjective mood (VASM), and their subjective driving performance. They underwent PVT at 10:30 (i.e., $3.5 \mathrm{~h}$ after waking up). The PSG and daily schedules were standardized across all subjects and both groups (insomnia patients and good sleepers).

\subsection{Sleep Recordings}

Sleep was recorded using an ambulatory PSG monitor (Medatec Dream). A standard montage of PSG was used, including eight electroencephalographic (EEG) channels (F3, F4, C3, C4, O1, O2, T3, T4, referenced on A1 and A2), two electro-oculogram (EOG) channels, and one submental electromyogram channel. The setup was complemented by recordings from the left and right anterior tibialis muscle, recordings of nasal/oral airflow, thoracic and abdominal effort, body position, and oximetry. All PSG were scored according to the standard criteria (196) by experienced sleep specialists (CC, FB).

Objective measures of sleep included SOL (min), wake after sleep onset (WASO, min), total sleep time (TST, min), sleep efficiency (SE, \%), total time (min), latencies $(\min )$, and percentage of stages $1,2,3-4$, and rapid eye movement (REM).

\subsection{Simulated Driving Test}

The driving experiment was carried out on the SIM2 INRETS fixed-base driving simulator equipped with an ARCHISIM object database (197). This simulator is composed of a steering cab connected to a personal computer for managing signal acquisition, sound restitution, and image display (Figure 1). The images, 
generated at a frequency of approximately $30 \mathrm{~Hz}$, were projected by a video projector onto a screen ( $\left.\mathrm{H}: 60^{\circ} ; \mathrm{V}: 49^{\circ}\right)$ located $1.90 \mathrm{~m}$ from the driver's eyes. The center of the screen was located at the driver's eye level $(1.38 \mathrm{~m})$. The acquisition frequency for the different signals (position, speed, acceleration, etc.) was $30 \mathrm{~Hz}$.

The monotonous driving test consisted of $1 \mathrm{~h}$ of driving in a driving simulator in monotonous conditions (no traffic, repetitive landscape). Participants were instructed to drive as straight as possible within the right traffic lane while maintaining a constant speed of $110 \mathrm{~km} / \mathrm{h}$. The primary parameter was the amount of weaving of the car, measured by the standard deviation of lateral position (SDLP, m), which is an index of safe driving or of road-tracking error. The SDLP was calculated using the lateral position data, and we first excluded all lateral position data that were outside the right traffic lane. Then, we calculated the mean lateral position and the standard deviation of this mean lateral position according to the mathematical definition of a standard deviation described in the study by Verster and Roth (198). The standard deviation of speed (SDS, km/h), the mean lateral position (mLP, m), mean speed $(\mathrm{mS}, \mathrm{km} / \mathrm{h})$, and the number of lane crossings (LC) were also analyzed.

We previously used the INRETS-FAROS simulator to evaluate monotonous driving performance after medication intake and showed that the simulator was sensitive enough to reveal driving impairments in monotonous conditions after drug intake (180).

\subsection{Vigilance Evaluation}

The PVT is a 10-min computerized task that assesses sustained attention and simple reaction time and requires continuous attention to detect randomly occurring stimuli $(32,98)$. The PVT requires acknowledgment of a visual stimulus (a white circle presented at the center of the screen) by pressing the response button. The response time was displayed on the screen after a subject's response. If the participant responded later than $500 \mathrm{~ms}$ or failed to respond within $1.5 \mathrm{sec}$, a "lapse" was recorded. Each stimulus was separated by a randomly determined interstimulus interval ranging from 3 to $10 \mathrm{sec}$. The number of lapses (n), the mean reaction time $(\mathrm{RT})(\mathrm{ms})$ and the mean slowest $10 \% 1 / \mathrm{RT}(\mathrm{ms})$ were 
quantified. A lapse was defined as a response slower than $500 \mathrm{~ms}$, in accordance with Drummond and colleagues (199). The variable "slowest 10\% 1/RT" was calculated according to the methodology described in Basner and Dinges. For calculating mean 1/RT and then the slowest 10\% 1/RT, each RT (ms) was divided by 1,000 and then reciprocally transformed. The transformed values were then averaged.

\subsection{Subjective Evaluations}

After the monotonous driving task, all participants rated their sleepiness with the KSS (121), their subjective feelings regarding change of mood with VAMS (194), and their "subjective driving quality", using a continuous scale for self-rating their driving quality (195) .

The KSS is a nine-point verbally-anchored scale with the following stages: (1) very alert; (3) alert; (5) neither alert nor sleepy; (7) sleepy; and (9) very sleepy, fighting sleep. Other points represent intermediate stages between the two neighboring points without definitions. This scale is often used to evaluate the acute sleepiness level (200).

The driving scale consisted of a 100-mm vertical visual analog scale with three levels: (1) "I drove exceptionally poorly" at $0 \mathrm{~mm}$; (2) "I drove normally" at $50 \mathrm{~mm}$; and (3) "I drove exceptionally well" at $100 \mathrm{~mm}$. Responses were given on a visual analog scale of $100 \mathrm{~mm}$ with an " $\mathrm{x}$ " marked at the appropriate level. If the response was near $\pm 50 \mathrm{~mm}$, the participants estimated that they drove normally and safely.

The VAMS comprised nine 100-mm lines anchored by antonyms (alert/drowsy, attentive/dreamy, muzzy/clear-headed, mentally slow/quickwitted, lethargic/energetic, well coordinated/clumsy, strong/feeble, interested/bored, proficient/incompetent). Participants were asked to place a mark on the horizontal line equivalent to the strength of a particular feeling at the given time before and after the driving task.

\subsection{Statistical Analysis}

Statistical analyses were carried out using R 2.15.0 software (www.r-project.org). The driving and VAMS data that were analyzed are of longitudinal structure with both random and fixed effects. To capture these effects, we selected linear mixed 
effects models as the methodological approach. To capture serial within-subject correlation, we investigated different correlation structures. Using the Bayesian information criterion (BIC), the time series typical AR(1) error structure was selected. Moreover, heteroscedasticity of the residuals was taken into account by the model structure. To evaluate whether a variable had a significant effect, we followed the approach of Pinheiro and Bates (201) and compared models with and without the respective variable by means of a likelihood ratio test (LRT). The statistical result of this model comparison is represented as the likelihood ratio L.r. value and the associated $\mathrm{P}$ value (e.g., L.r. $=\mathrm{XX}$; $\mathrm{P}=\mathrm{XX}$ ). When the LRT indicated a significant effect of a variable, the coefficients of the model were further examined and represented as the $t$ and $p$ values associated with each variable tested (e.g., $\mathrm{t}(35)=\mathrm{XX} ; p=\mathrm{XX})$. For analyses with several variables of interest, these were included stepwise into the model: starting with the variable subject to the strongest effect, and successively adding those with weaker effect.

For driving data, the choice of comparing the first 20 to the last $30 \mathrm{~min}$ via a dummy variable was taken after visual inspection of the data, which suggested that the two groups progressed differently over time. As the main effect manifests itself via a constant increase in the variable considered during the last $30 \mathrm{~min}$, the modeling approach via a dummy variable seemed natural from a statistical point of view. Therefore, we added a dummy variable for the last $30 \mathrm{~min}$ of driving in interaction with the group membership.

For comparisons of two (unpaired) groups, the $t$-test and Welch test were used if the normality hypothesis could not be rejected by the Shapiro-Wilk test. For non-Gaussian samples, preference was given to the Wilcoxon-Mann-Whitney test.

Finally, the relationship of the main parameter of driving (SDLP) with the main sleep variables (TST, SE) was evaluated with tests for correlation, and $2 \times 2$ frequency tables were analyzed by Fisher's exact test.

For all statistical methods applied, we report the respective effect sizes. That is, for comparison of two groups we provide Cohen's d (149) and Cliff delta (202). Cliff's delta is more appropriate for samples violating the normality assumption, whereas Cohen's d should be considered in the gaussian case. The effect size calculated for $2 \times 2$ frequency tables is the phi coefficient (203), and for 
mixed effects models we used partial and conditional $\mathrm{R}^{2}$, introduced by Nakagawa and Schielzeth (204). The level of statistical significance was set at $p<0.05$.

\section{Results}

After PSG screening, five of 42 participants were excluded because of other sleep disorders (e.g., sleep apnea or periodic limb movement), three of them were in the insomnia group and two in the good sleepers group. Twenty-one patients with insomnia and 16 good sleepers were included in the final analyses (Table 1).

\subsection{Sleep Recordings}

Participants with insomnia had significantly lower SE $(p=0.048)$ and a longer duration of awakenings after sleep onset $(p=0.021)$ compared to good sleepers. Patients with insomnia and good sleepers did not differ in the other sleep parameters (Table 2). 


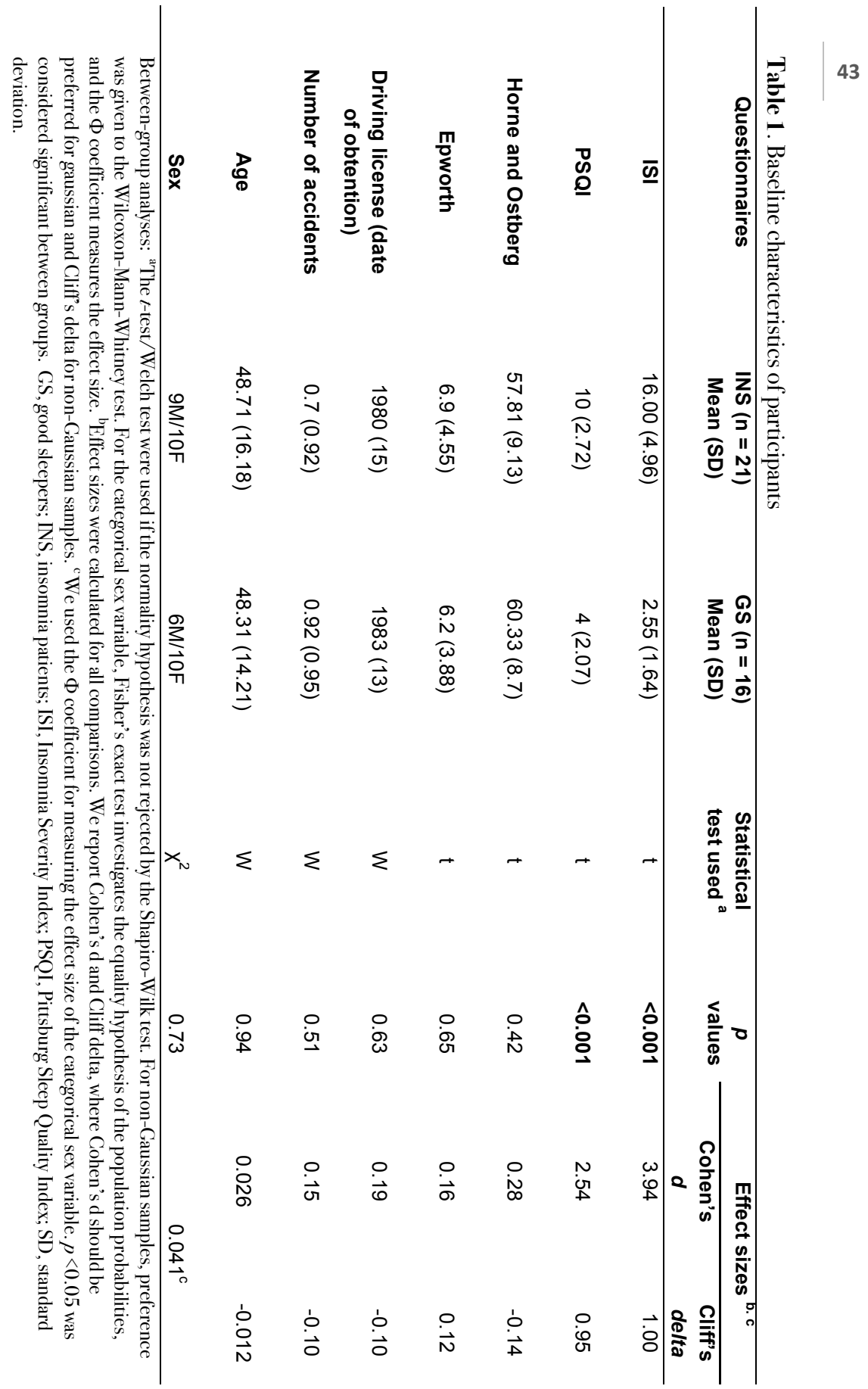




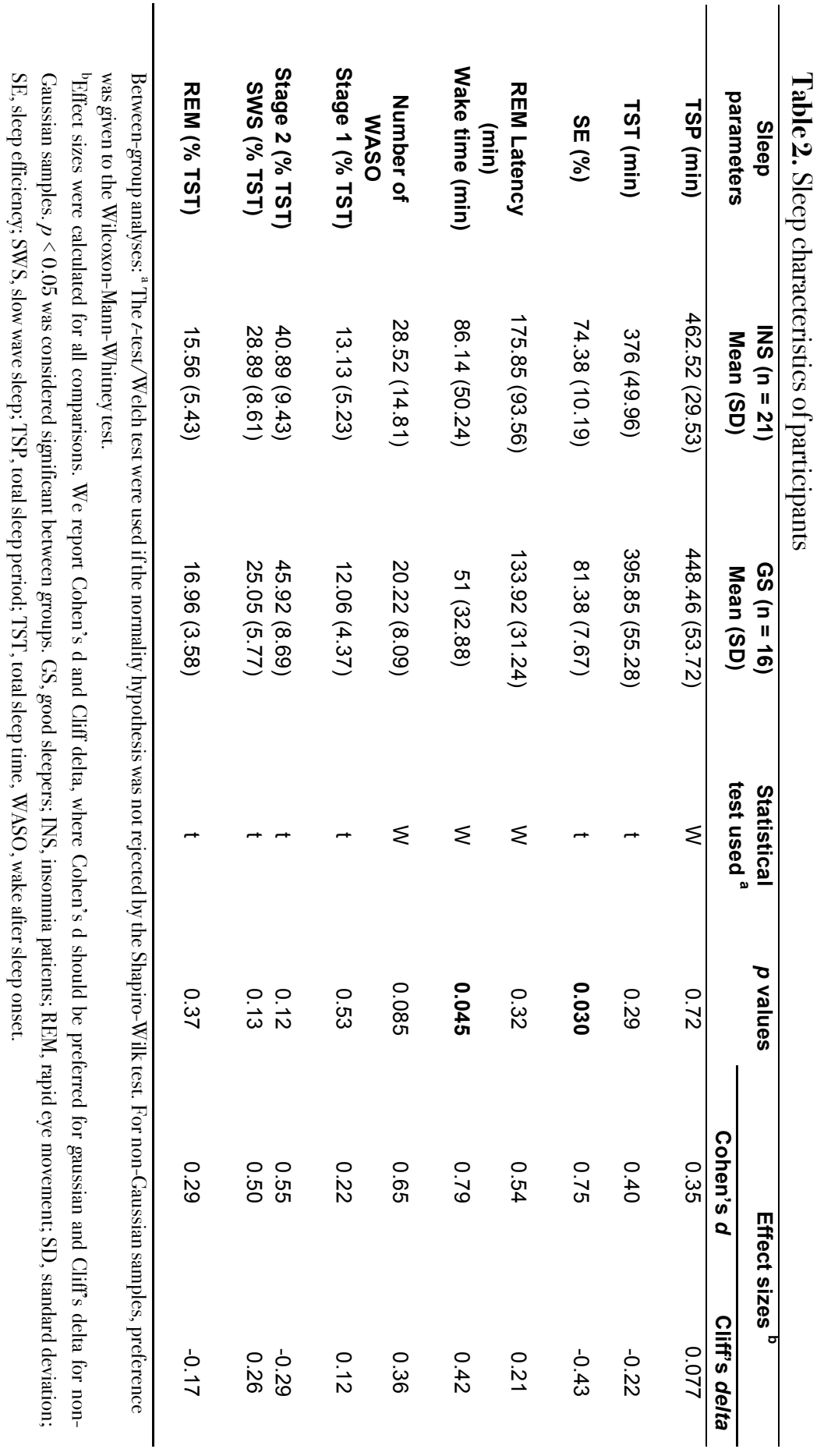




\subsection{Monotonous Driving Test}

Results are presented in Table $3 \mathrm{a}$ and $\mathrm{b}$, and the electronic supplement contains a table with more detailed statistical results of the mixed effects model estimation used for the main driving parameters.

Analysis of the primary parameter, the SDLP variable, showed a significant group effect (L.r. $=5.34 ; \mathrm{P}=0.021$ ). The model coefficient indicated that participants with insomnia had a significantly greater SDLP than good sleepers $\left(\mathrm{t}_{(35)}=2.38 ; p=0.023\right)$. The resulting division of the test into two periods (the first $20 \mathrm{~min}$ and last $30 \mathrm{~min}$ ) using the dummy variable is supported by the LRT (L.r. $=7.55 ; p=0.023$ ). Investigation of the model coefficients revealed a significant difference between the two periods for the insomnia group $\left(\mathrm{t}_{(146)}=2.70 ; p=0.008\right)$. However, no significant difference between the two periods could be detected in the good sleepers group $\left(\mathrm{t}_{(146)}=-0.53 ; p=0.60\right)$. These results indicate that the insomnia group is subject to a significant increase in the SDLP after 20 min of driving.

Similarly, analyses of data on the number of lane crossings showed a significant effect of both the group variable (L.r. $=4.82 ; p=0.0282$ ) and the aforementioned dummy variable effect (L.r. $=13.89 ; p=0.001$ ). The model coefficient indicated a significant difference between patients with insomnia and good sleepers $\left(\mathrm{t}_{(35)}=2.2 ; p=0.030\right)$. Moreover, participants with insomnia showed a significant increase in the number of lane crossings $\left(\mathrm{t}_{(146)}=3.03 ; p=\right.$ 0.003 ) during the last $30 \mathrm{~min}$, whereas a smaller but significant decrease in the number of lane crossings occurred in the good sleepers group $\left(\mathrm{t}_{(146)}=-0.26 ; p=\right.$ 0.025) (Table 3 and Figure 2).

Significant effects of the dummy variable are indicated on the graph. The dummy variable represents the comparison between the period of the first $20 \mathrm{~min}$ and the period of the last 30 minutes of driving for each group. $\mathrm{P}<0.05$ was considered significant between periods. No significant group effect was found for the SDS $(p=0.617)$, the lateral position $(p=0.061)$, or the $\mathrm{mS}(p=0.646)$. These three parameters were used as controls to ensure that participants respected the driving instructions (Table $3 \mathrm{~b}$ ). 


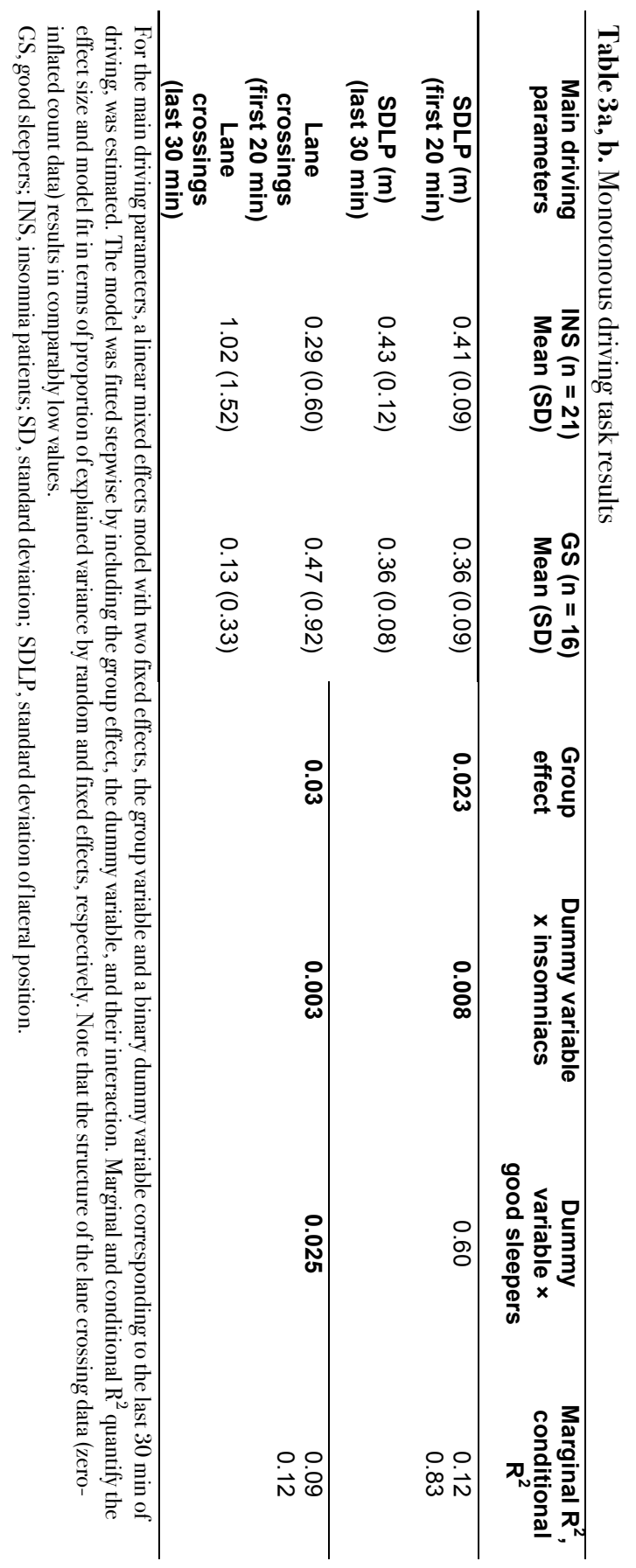




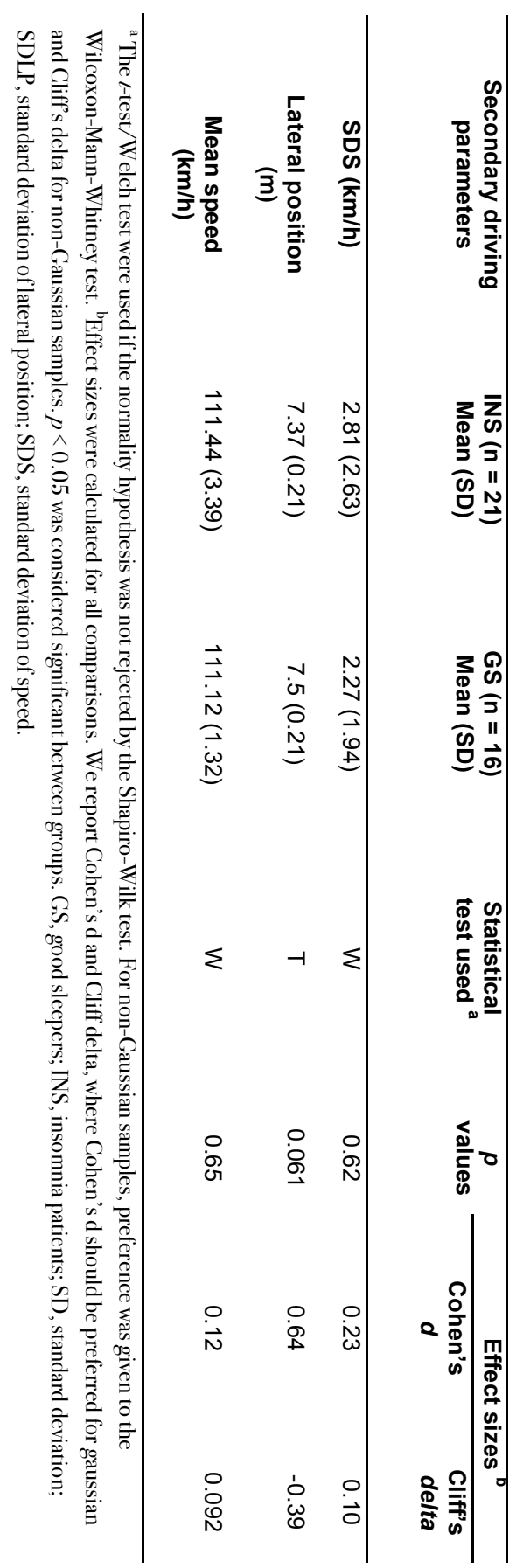




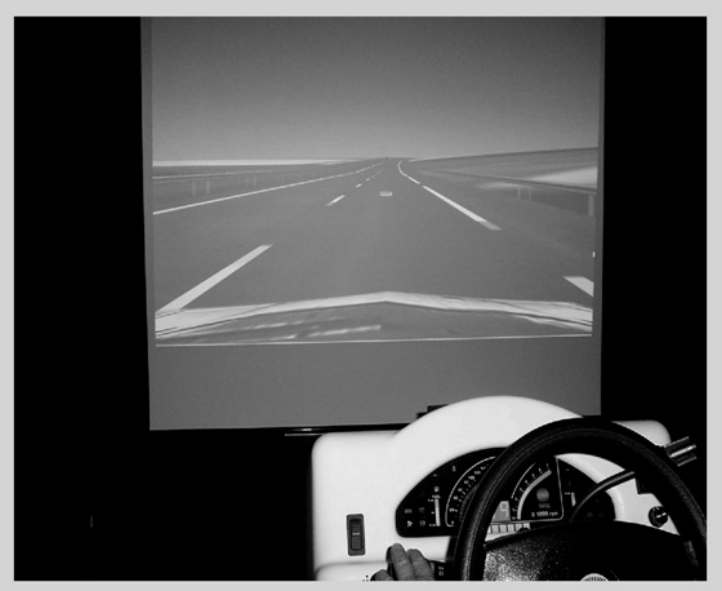

Figure 1. Example of the visual interface of the simulator used for the experiment.

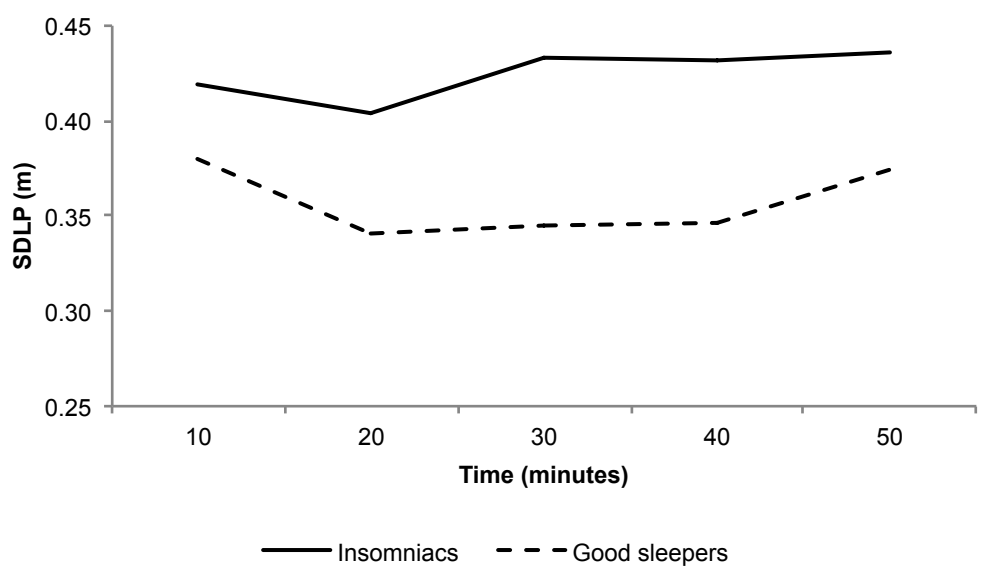

Figure 2. Standard deviation of lateral position (SDLP) throughout the driving task. 


\subsection{Self-Assessment Scales}

Results are presented in Table $4 \mathrm{a}$ and $\mathrm{b}$, and the electronic supplement provides a table with details on the mixed effects model used for the VAMS.

Patients with insomnia evaluated their own driving performance as accurately as good sleepers $(p=0.15)$. Results of the sleepiness scale revealed a nonsignificant trend in group effect; insomnia patients felt less awake than good sleepers $(p=0.060)$ (Table 4a). For the VAMS data analyses with a mixed model, group and time of testing revealed significant effects of group (L.r. $=7.64 ; p=$ 0.006 ) and session (L.r. $=4.03 ; p=0.045$ ). The coefficients of the model showed that the patients with insomnia had a lower alertness score than the good sleepers $\left(\mathrm{t}_{(36)}=2.87 ; p=0.007\right)$; participants with insomnia felt less alert than good sleepers. Moreover, the model coefficients revealed a significantly higher alertness score for the session before driving than for the session after driving; the participants felt less alert after driving than before driving $\left(\mathrm{t}_{(35)}=2.10 ; p=0.043\right)$ (Table 4b).

\subsection{Psychomotor Vigilance Test}

Analyses of the PVT data showed no group effect for all variables analyzed (Table 5).

\subsection{Correlation Between Driving and Sleep Data}

Coefficients of correlation revealed a significant negative effect of TST ( $p$ $=0.011)$ and $\mathrm{SE}(p=0.009)$ for predicting the weaving of the car (SDLP), but no significant effect for the duration of WASO. When TST or SE decreases, SDLP increases, thus driving performance decreases (Table 6). 
Chapter 3

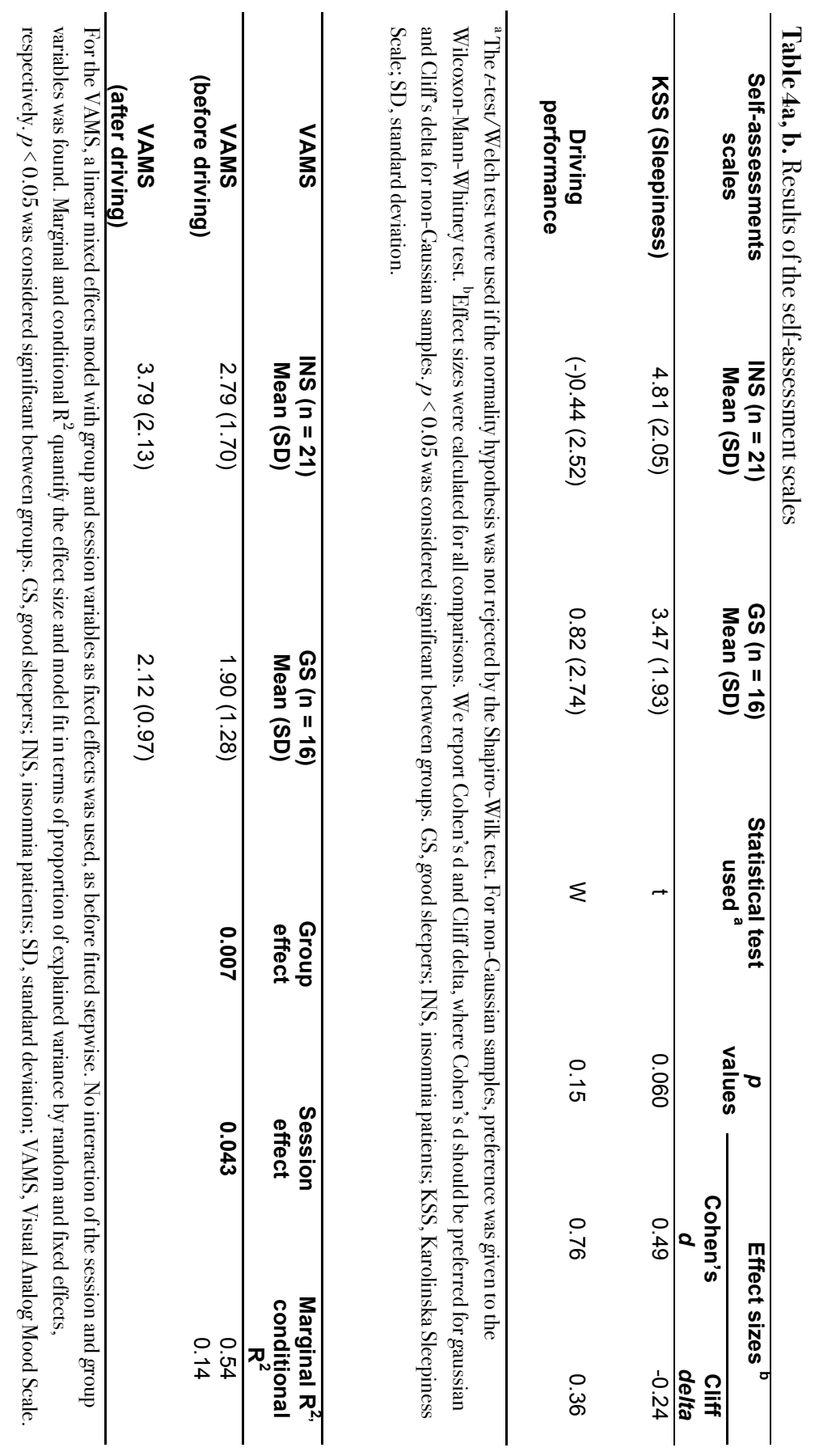




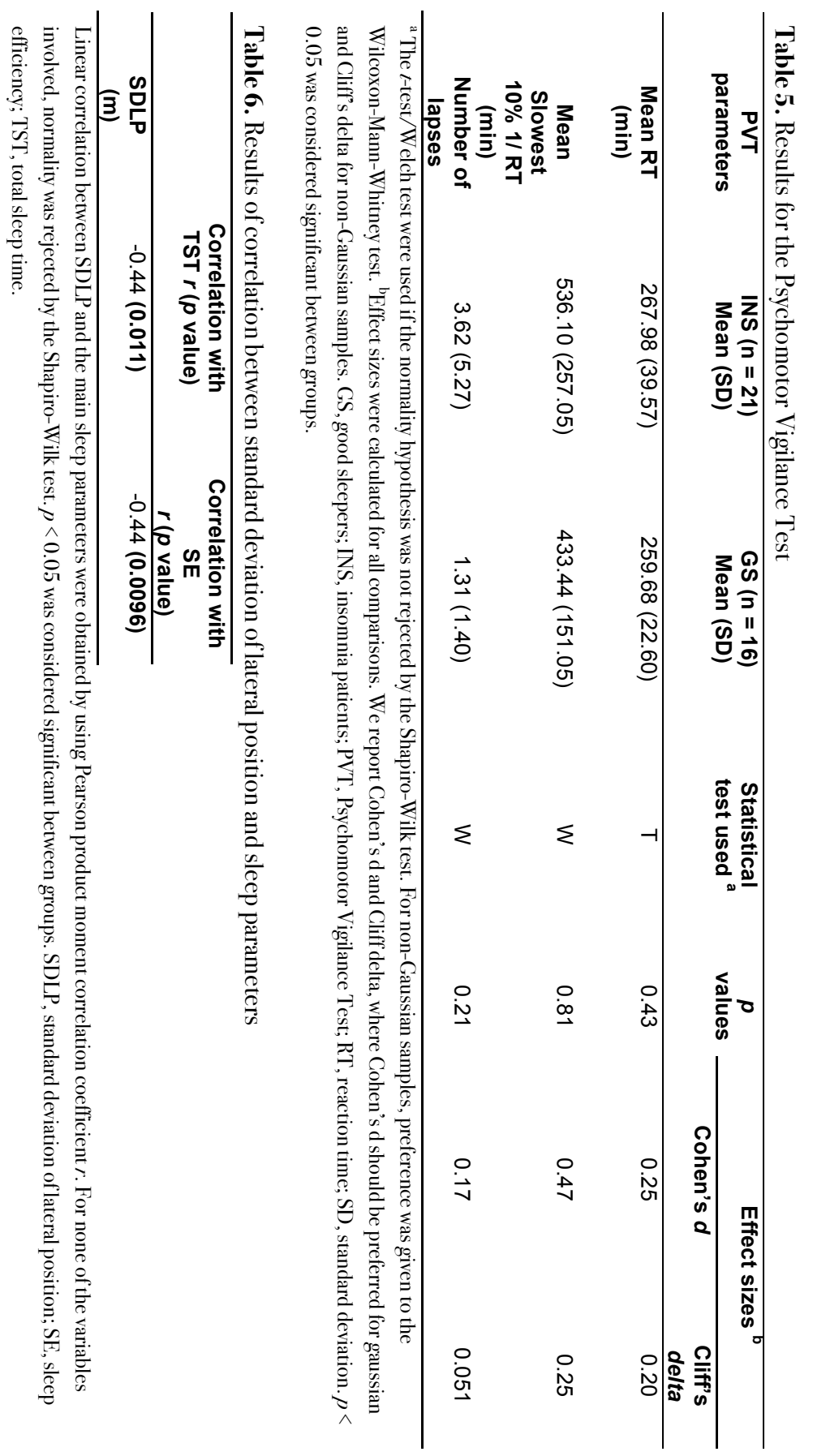




\section{Discussion}

This study was primarily aimed at evaluating the driving performance of untreated patients with primary insomnia in comparison with good sleepers. Our results revealed that patients with primary insomnia had impaired simulated monotonous driving performance compared to good sleepers, as shown by the increase in SDLP and the number of road exits. These findings indicate that patients with primary insomnia experienced more difficulty in maintaining the trajectory of the simulated vehicle than did good sleepers. In terms of traffic safety, each driving lane crossing could lead to a loss of trajectory control and thus to a potential accident. The impaired simulated driving performance in the insomnia group appeared to be in agreement with an epidemiological study showing that patients with insomnia had approximately three times more risk of having traffic crashes than controls (176). However, in this epidemiological study (176), no specific analysis was performed between treated and untreated patients with insomnia. The current study is the first to reveal that simulated monotonous driving performance is impaired in patients with primary insomnia. Moreover, the current findings also revealed that impairments occurred during the last $30 \mathrm{~min}$, both for the SDLP and the number of lane crossing parameters, which indicates that patients with insomnia were no longer able to perform as well as good sleepers after $20 \mathrm{~min}$ of simulated driving.

Although the values of SDLP in the current study are higher than those usually found in on-the-road driving studies (205), they are in agreement with values reported in several studies using simulators $(206,207)$. The difference in SDLP values can be explained by several methodological characteristics inherent to the simulators such as the field of vision, the force feedback of the steering wheel, or the type of road (straight line or curves). In addition, the experimental conditions between on-the-road driving and simulators studies are quite different in terms of awareness of danger, light exposure, proprioceptive feedback, and stimulating environment. We may hypothesize that a simulated environment has facilitated the detection of an impaired driving performance in the untreated group with insomnia. Nevertheless, this has no influence on the comparison between the insomnia and control groups because only the performance 
difference between both groups has a significant meaning, and both groups completed the simulated driving task in the same experimental conditions.

The secondary objective of this study was to assess performance of sustained attention by using the PVT, which is sensitive to sleep pressure $(32,98,99,208)$. The lack of impairment found in the insomnia group is in agreement with previous studies that used similar simple short duration reaction time tasks (68). Orff and colleagues (74) used the same PVT as that used in the current study, and did not find any significant impairment although their sample size was larger than ours (32 insomnia patients and 17 good sleepers). In comparison, performance impairments in insomnia patients were observed only if complex short duration reaction time tasks were used $(69,71)$. The effect sizes reported for the PVT in the current study were small to medium, which may indicate that a larger sample size might be required to render the differences statistically significant. We subsequently carried out a Monte Carlo approach to check which sample size would have been required to obtain a sufficient level of power (0.8). The results obtained show that very large sample sizes are required (more than 90 participants for the variable number of lapses, which is the smallest sample size required among all variables). Thus, our findings indicate that both groups are subject to very small differences and that the PVT may not be the best method for highlighting cognitive dysfunction in insomnia.

The lack of impairment in the PVT in the current study is also in agreement with the lack of impairment observed during the first $20 \mathrm{~min}$ of the monotonous driving test, suggesting that patients with insomnia are able to maintain the same performance level as good sleepers during tasks of relatively short duration with low cognitive demand. Although the monotonous motorway driving test used in the current study is probably more complex than the PVT, it involves automatic processing and requires a low attentional demand (209). These findings revealed that patients with insomnia are able to adequately perform a relatively simple task of short duration, but are not able to maintain their level of performance when the task is longer, even if it is simple. We hypothesized that patients with insomnia probably compensate their effort during the first 20 min of the driving task and during the PVT. This compensatory effort is probably because 
of the hyperarousal phenomenon $(80,81)$, which masks impairment when short or simple tests are used.

The subjective measures were used to characterize variations in driver performance and alertness level. The KSS measures, although significantly different between the two groups, did not show patients with insomnia to be sleepy. They only felt slightly less awake than good sleepers, which is in agreement with a previous study (67). The VAMS revealed that the level of alertness was lower after the driving task in comparison to before for both groups, which is probably linked to the long duration of the driving task. Moreover, patients with insomnia felt less alert than good sleepers both before and after the driving test. It is interesting to observe that the level of alertness was on average more changed in the insomnia patients group than in the good sleepers group after the driving test. Although this interaction did not reach significance level, this result suggests that the monotonous driving test required more effort for the patients with insomnia than for the good sleepers. No correlation was found between the insomnia severity index and both objective and subjective measures of driving performance (data not shown). However, the subjective scale of driving performance revealed that the patients with insomnia seemed to be unaware of their driving difficulties because they estimated their driving performance as normal, as did good sleepers. This dissociation between objective evaluation and subjective perception is an important concept in traffic safety because drivers can overestimate their own ability to drive safely.

Sleep analysis showed that patients with insomnia objectively slept worse than did good sleepers in the laboratory, which confirmed subjective complaints measured by the PSQI. Findings on sleep architecture in primary insomnia are inconsistent $(79,210)$. Nevertheless, our results demonstrate that the simulated driving impairment was associated with both poor SE and quantity; the worse participants slept, the worse they drove. Interestingly, this link was found for both groups, with no intergroup differences observed. This result implies that the simulated driving performance depends on the quantity and the efficiency of sleep, independent of the participant status (being a patient with insomnia or a good sleeper). This relation between sleep quality and daily performance is thus in line with the study by Fernandez-Mendoza and colleagues (211) which reported that 
only patients with insomnia with short sleep duration had objective cognitive dysfunction, which was not found in patients with insomnia with normal sleep duration.

Although the wide age range of the participants may be a limitation of the current study, no age effect was found in any parameter. However, a limitation of the study is the relatively small sample size of participants that may have prevented us from being able to assess whether young persons with insomnia experienced more impairments than older persons, as suggested by another study (212).

Because most patients with insomnia are treated with hypnotics, further research is needed to study this population to assess whether the treatment has beneficial or deleterious effects on driving performance. This question remains to be addressed, as it has been clearly shown that hypnotics impair driving performance after a single intake in healthy young or older subjects $(178,180)$, or after $1 \mathrm{w}$ of treatment in patients with insomnia (177), but the effects of long-term treatment with hypnotics have not yet been studied.

In conclusion, the current study revealed that primary insomnia was associated with impaired driving performance during a simulated monotonous driving task. We also demonstrated that patients were able to drive for very short periods (less than $20 \mathrm{~min}$ ), but when the duration of the task was longer, impairment occurred (probably because of decreased vigilance). The current findings highlight complaints expressed by patients regarding performance of daily tasks. It appears advisable for clinicians to warn patients about their impaired driving performance that could lead to an increased risk of driving accidents, particularly if they have to drive for relatively long periods of time in a monotonous environment. In addition, the current findings indicate that the driving impairments appeared to be associated with poor sleep efficiency and quantity without distinction between groups. 



\section{Cortical activity modifications in primary insomnia and after sleep deprivation}





\section{Chapter 4 \\ Electroencephalography during \\ on-the-road driving in older untreated insomnia patients and normal sleepers}

Published as:

Perrier, J., Leufkens T., Bulla J., Jongen S., Bocca, M.-L., Ramaekers, J. G., \& Vermeeren, A. (2015). Electroencephalography during on-the-road driving in older untreated insomnia patients and normal sleepers. Biological Psychology, $109,20-28$. 


\begin{abstract}
Insomniacs report decreased performance in daily routines, which may have detrimental consequences for car driving. We compared changes over time in driving performance (measured as Standard Deviation of Lateral Position - SDLP) and background EEG between 20 untreated insomnia patients (52-70 years old) and 21 normal sleepers (54-73 years old) during a one hour on-the-road driving test after a normal night of sleep, in the morning. SDLP did not differ between groups and increased slightly over time to similar degrees in both groups. EEG alpha and beta power were lower in insomniacs as compared to normal sleepers. Alpha and beta power slightly reduced during driving in normal sleepers but remained at a constant low level in insomniacs. Changes in EEG power and SDLP were not related. It is concluded that on-the-road driving performance does not differ between older insomniacs and older normal sleepers and that changes in spectral EEG measures of cortical arousal and in driving performance are not related.
\end{abstract}




\section{Introduction}

Insomnia is one of the most common complaints seen in medical practice. Approximately one-third of the general population experiences some insomnia symptoms occasionally, and it is estimated that approximately $10 \%$ suffers from chronic insomnia $(5,6)$. Insomniacs report reduced performance in daily routines, which may have detrimental consequences in particular for potentially hazardous activities such as car driving. A number of studies have shown that the consequences of sleep problems include decreased work productivity, increased risk for accidents, increased health care costs $(11,13,20,213)$. Specific impairment of driving performance and increased risks for traffic accidents has been shown for sleep disturbances, such as obstructive sleep apnea, periodic limb movement disorder and sleep deprivation (e.g. $(20,51,214,215)$. The link between insomnia and driving impairment is less clear, however $(11,24,176,215,216)$. Few epidemiological studies have found increased traffic accident risks in insomnia patients $(217,218)$, while several others do not $(24,215,219,220)$. The inconsistent findings in epidemiological research are in line with those of experimental research. Experimental studies have generally failed to establish objective evidence of cognitive dysfunction, despite insomnia patients' subjective feelings of fatigue and cognitive impairments $(67,68)$.

Recently, two studies comparing driving performance of insomnia patients to that of normal sleepers, found significant impairment in a driving simulator task, but no differences in an on-the-road driving test $(144,221)$. Leufkens and colleagues (221) compared sleep and performance between a group of 20 older untreated insomnia patients, a group of 22 chronic users of hypnotics and a group of 21 age-matched normal sleepers, using a one-hour on-the-road highway driving tests and a battery of tests measuring driving related cognitive and psychomotor performance. Results showed no significant differences between groups in performance, despite significant sleep complaints in the insomnia patients. In contrast, Perrier and colleagues (144), using a driving simulator, found that insomnia patients performed worse than normal sleepers. The impairment found in insomnia patients seemed to be due to a performance decrement in the second half of the test, i.e. after 20 minutes of monotonous driving on a highway. This 
suggests that insomnia may show more pronounced vigilance decrements than normal sleepers during long and monotonous tasks, such as a simulated highway driving task.

The lack of impairment during real driving in the study by Leufkens and colleagues (221) raises the question whether this may have been due to hyperarousal in patients. Current models of primary insomnia assume that patients are hyperaroused or unable to de-arouse, which would account for their difficulties in initiating and maintaining sleep but also for the absence of daytime sedation and associated performance impairment (80,81). Several studies have looked at EEG markers of arousal during sleep in insomnia patients and have found increased power in the beta and sigma frequency band around sleep onset and during NREM sleep, reflecting increased cortical activation in patients as compared to normal sleepers ((79) for review). Only two studies have been published evaluating daytime waking EEG in insomnia patients. In line with findings during sleep, Wolynczyk and Szelenberger (82) report increased beta power during daytime Multiple Sleep Latency Tests, whereas Wu and colleagues (83) report no significant differences between patients and normal sleepers in 5minutes resting EEG recordings with eyes open and eyes closed. It is therefore not clear whether EEG in insomnia patients during task performance such as driving will show changes as indication of increased arousal.

A few studies evaluated background EEG during on-the-road driving in healthy volunteers and found that brain activation decreases (i.e. increases in theta and alpha power spectra) with time-on-task $(222,223)$, during night-time driving $(65,224,225)$, and after sleep deprivation (226). Similar changes in EEG have been reported by investigators using driving simulators (e.g. $(52,135,137,227,228)$. EEG changes such as increases in alpha and theta activity, and decreases in beta activity, are often associated with decreased alertness, increased drowsiness and worse performance (125). Until now, no study compared EEG during driving between insomnia patients and normal sleepers.

The purpose of the present study was to assess whether changes in spectral EEG measures are related to changes in driving performance in older insomnia patients. It was expected that insomnia patients on average show 
increased cortical arousal during driving as compared to normal sleepers, in particular more beta power, and less alpha and theta power. It was also expected that insomniacs do not display increased indicators of fatigue and sleepiness (i.e. theta and alpha); contrary to normal sleepers. In addition it is expected that impaired driving in patients is associated with reduced beta power, rather than increased alpha or theta power compared to normal sleepers.

\section{Methods}

\subsection{Design}

Data for this paper were collected as part of study that compared sleep, on-theroad driving performance and driving related skills between older untreated insomnia patients, chronic users of hypnotics, and age-matched normal sleepers (221). For the present study EEG recordings during driving were analyzed and compared between two groups, i.e., untreated insomnia patients and normal sleepers.

\subsection{Participants}

The insomnia group comprised 20 healthy older adults (10 men, 10 women; 52 70 years old; mean \pm SD age $60.8 \pm 5$.8 years) with insomnia, and not using sleep promoting agents for more than 3 nights per week. The control group comprised 21 normal sleepers (13 men, 8 women; 54-73 years old; mean \pm SD age 61.7 \pm 5.0 years). Participants were recruited via advertisement in local newspapers and through a network of local general practitioners in the region of Maastricht.

All participants had to meet the following inclusion criteria: aged between 50 and 75 years; possession of a valid driving license; average driving experience of at least $3000 \mathrm{~km}$ per year over the last 3 years; good health based on a pre-study physical examination, medical history, vital signs, electrocardiogram, blood biochemistry, haematology, serology and urinalysis; body mass index (BMI) between 19 and $30 \mathrm{~kg} / \mathrm{m} 2$. Exclusion criteria were history of drug or alcohol abuse; presence of a significant medical, neurological, psychiatric disorder, or sleep disorder other than insomnia; chronic use of medication that affects driving performance; for patients, use of hypnotics for more than 3 nights per week; 
drinking more than 6 cups of coffee per day; drinking more than 21 alcohol containing beverages per week; smoking more than 10 cigarettes per day.

Insomnia patients had to meet the criteria for primary insomnia according to DSM IV (229): (a) subjective complaints of insomnia, defined as difficulties initiating sleep (sleep latency $>30 \mathrm{~min}$ ) and/or maintaining sleep (awakenings $>30 \mathrm{~min}$ ); (b) duration of more than 1 month; (c) the sleep disturbance causes clinically significant distress or impairment; (d) insomnia does not occur exclusively during the course of a mental disorder and (e) insomnia is not due to another medical or sleep disorder or effects of medication or drug abuse. Participants in the control group were self-defined normal sleepers. Sleep complaints and psychopathology were evaluated by a trained psychologist using Dutch versions of validated questionnaires. In addition, sleep was evaluated during two nights of polysomnography; first during a habituation night and next during the night before the driving test. Self-report data on sleep showed that insomniacs rated their sleep significantly worse than normal sleepers (221). Polysomnography did also not reveal significant differences between groups in sleep duration or architecture (221).

During participation, use of caffeine was prohibited from 8 hours prior to arrival on test days, until discharge the next morning. Alcohol intake was not allowed from 24 hours prior to bedtime until discharge. Smoking was prohibited from 1 hour prior to bedtime until discharge. Insomnia patients were not allowed to take hypnotic medication the night before driving.

The study was conducted in accordance with the code of ethics on human experimentation established by the World Medical Association's Declaration of Helsinki (1964) and amended in Edinburgh (2000). The protocol was approved by the medical ethics committee of Maastricht University and University Hospital of Maastricht. Participants were explained the aims, methods, and potential hazards of the study and they signed a written informed consent prior to any studyrelated assessments.

\subsection{Procedure}

All participants completed two nights of sleep evaluation and testing. The first night was a habituation and practice condition to familiarize participants with the 
sleeping facilities, polysomnography and test procedures, including a complete dress-rehearsal of the driving test. The second night was for actual sleep and performance assessments. Test conditions started in the evening when participants arrived at the site at $7.00 \mathrm{pm}$. Electrodes for polysomnographic recording were attached at $9.00 \mathrm{pm}$ and participants retired to bed at $11.30 \mathrm{pm}$. The following morning participants were awakened at 7:30 am by an experimenter and served a standardized light breakfast. At 9.00 am they were transported to the highway to perform the on-the-road driving test between 9.30 and $10.30 \mathrm{am}$. Upon completion participants returned to the testing facilities for removal of the electrodes and were discharged. All participants completed the study between December 2007 and February 2009. Normal sleepers were enrolled largely in parallel with insomnia patients, with only a few weeks delay to allow matching for age and gender of the control group.

\subsection{Assessments}

On-the-road highway driving test - Driving performance was assessed using a standardized on-the-road highway driving test (108) which measures road tracking performance. In this test, participants operate a specially instrumented vehicle for approximately one-hour over a 100-km (61 miles) primary highway circuit in normal traffic, accompanied by a licensed driving instructor having access to dual controls (brakes and accelerator). The participants' task is to drive with a steady lateral position between the delineated boundaries of the slower (right) traffic lane, while maintaining a constant speed of $95 \mathrm{~km} / \mathrm{h}(58 \mathrm{mph})$. Participants may deviate from those instructions only to pass a slower vehicle, and to leave and re-enter the highway at the turnaround points. During the drive, the vehicle's speed and lateral distance to the left lane line are continuously recorded. These signals are digitized at a rate of $4 \mathrm{~Hz}$ and stored on an onboard computer for later preprocessing and analysis. The signals are edited off line to remove data recorded during overtaking maneuvers or disturbances caused by roadway or traffic situations. The remaining data are then used to calculate means and standard deviations of lateral position and speed For the present study, the dataset was split in 6 successive periods of 10 minutes in order to analyze performance 
changes with Time-on-Task (ToT). The primary outcome variable is SDLP in cm, which is an index of weaving.

Electroencephalography - EEG was recorded with a Vitaport portable EEG recorder at four electrodes (F4, C4, O2 and C3) with a common average (A1-A2). EEG was continuously recorded during the driving test. Data were sampled at 256 $\mathrm{Hz}$ and was low pass filtered at $70 \mathrm{~Hz}$. In addition, a $50 \mathrm{~Hz}$ notch filter was applied to remove artifacts linked to electrical activity in the car. After segmentation of data into two-second epochs data were visually inspected for artifacts that were subsequently rejected. Each epoch was then subjected to a fast Fourier transformation using a Hanning window of $10 \%$ in order to obtain the power spectrum. The following band frequencies were analyzed and quantified in absolute power for 6 consecutive 10-minute periods of the driving test: theta (4-8 $\mathrm{Hz})$, alpha $(8-13 \mathrm{~Hz})$ and beta $(13-30 \mathrm{~Hz})$.

EEG recordings of 9 participants were not included in the analysis: 3 participants had no valid EEG data due to technical problems during recording, data from 3 participants could not be used because of too many movement artefacts, and data from 3 participants were excluded because of outliers (mean $\pm 2 \mathrm{SD})$. In total EEG data sets were analyzed for 32 participants: 17 insomnia patients ( 8 men, 9 women; $55-70$ years old mean \pm SD age $61.6 \pm 5.8$ years $)$ and 15 normal sleepers ( 7 men, 8 women; $54-68$ years old; mean \pm SD age $60.7 \pm 4.8$ years). Driving data were analyzed for 34 participants: 17 insomnia patients $(9$ men, 8 women; 54-70 years old; mean \pm SD age $61.41 \pm 5.98$ years) and 17 normal sleepers ( 9 men, 8 women; $55-68$ years old; mean \pm SD age $61.60 \pm 4.30$ years). For five participants driving data were omitted from analysis, because there were no corresponding EEG data. For two participants the datafiles could not be retrieved for analysis. Analysis of a potential linear relationship between driving performance and EEG was conducted on participants for which both driving and EEG data were available i.e 30 participants including 16 insomnia patients ( 7 men, 9 women; $55-70$ years old; mean \pm SD age $61.88 \pm 5.85$ years) and 14 normal sleepers ( 6 men, 8 women; $55-68$ years old; mean \pm SD age $61.14 \pm 4.57$ years). 


\subsection{Statistical analysis}

Statistical analyses were carried out using the software R 2.15.0 (www.rproject.org). The driving and EEG data that were analyzed are of longitudinal structure with both random (participant) and fixed (Group, ToT) effects. To capture these effects, we selected linear mixed effects models as methodological approach. More precisely, the models analyzed are of ANCOVA type. That is, in case of full interaction the basic model form is:

$$
\text { Pow }_{E E G}=\beta_{0}(G r)+b+\beta_{1}(G r) \cdot T o T+\varepsilon,
$$

where $P o w_{E E G}$ denotes the response variable, $\beta_{0}$ the group-varying intercept, $\beta_{1}$ the group-varying slope coefficient, ToT the time-on-task covariate, and $\varepsilon$ the error term, following a Gaussian distribution with expected value zero. In addition to a classical ANCOVA, the term $b$ represents a Gaussian random effect and captures between-subject variability in the intercept. The model would also allow for the inclusion of random effects in the slope coefficient, which proved not to be necessary for obtaining a better model fit.

Mixed effects models are versatile, and able to take different data characteristics into account. In order to build models suited to the different data sets analyzed, we carried out the following steps:

1. As a preliminary step, we investigated the residuals of various models. It turned out that serial within-subject correlation was present for most series. Therefore, we investigated different correlation structures of the error term $\varepsilon$. Taking the Bayesian information criterion (BIC) as selection criterion, the for time series typical $\mathrm{AR}(1)$ error structure was selected if indicated by the criterion. Moreover, the correlation structure was verified on the finally selected model for each data set.

2. Following a similar model selection approach as described in the first point, we investigated different variance models accounting for potential heteroscedasticity of the error term $\varepsilon$. The variance functions investigated were: fixed variance, different variances for normal sleepers and insomniacs, ToT-varying variance, as well as group- and ToT-varying variance. The most appropriate variance structure was selected by means of the BIC. 
3. When building a model, one has to evaluate if a variable entering a model has a significant effect. We followed the approach of Pinheiro \& Bates (201), and compared models with and without the respective variable by means of a likelihood ratio test (LRT). To do this, the starting point for each analysis always was the simplest model, including only a constant intercept $\beta_{0}$ and random effect $b$. Then, we added the strongest fixed effect (e.g., time-on-task, resulting in a slope potentially unequal to zero) to the model and checked if the LRT justifies the inclusion of this additional variable. If so, we continued by adding the next variable (e.g., the group effect), either additively (i.e., affecting the intercept) or in interaction with the previously included variable (i.e., affecting the slope), and compared the resulting models by LRT and BIC, until the best model was selected.

4. After carrying out the previous steps for all data sets, and determining the best model for each, we accounted for the effect of multiple comparisons: the pvalues of the LRT of the final models were adjusted by the Holm-Bonferroni method. These results are represented by the associated $p^{\mathrm{LRT}}$ value.

5. Only when the LRT a model indicated a significant effect of one or several variables (ToT, group, or ToT and group interaction), the p-values of the coefficients $\left(\beta_{0}, \beta_{1}\right)$ of this model were further examined.

Potential dependencies between EEG and SDLP data were investigated by treating both variables in a linear mixed effects model similar to the one previously described. In this case, the model equation was

$$
S D L P=\beta_{0}(G r)+b+\beta_{1}(G r) \cdot T o T+\varepsilon,
$$

that is, only the response variable changed.

For all figures representing longitudinal data, we applied the approach described by Cousineau (230). Consequently, error bars in these figures do not take variability associated with between-subject differences into account. 


\section{Results}

\subsection{Driving performance}

Mean SDLP scores in both groups for 6 consecutive 10-minute intervals of the driving test are presented in Figure 1.

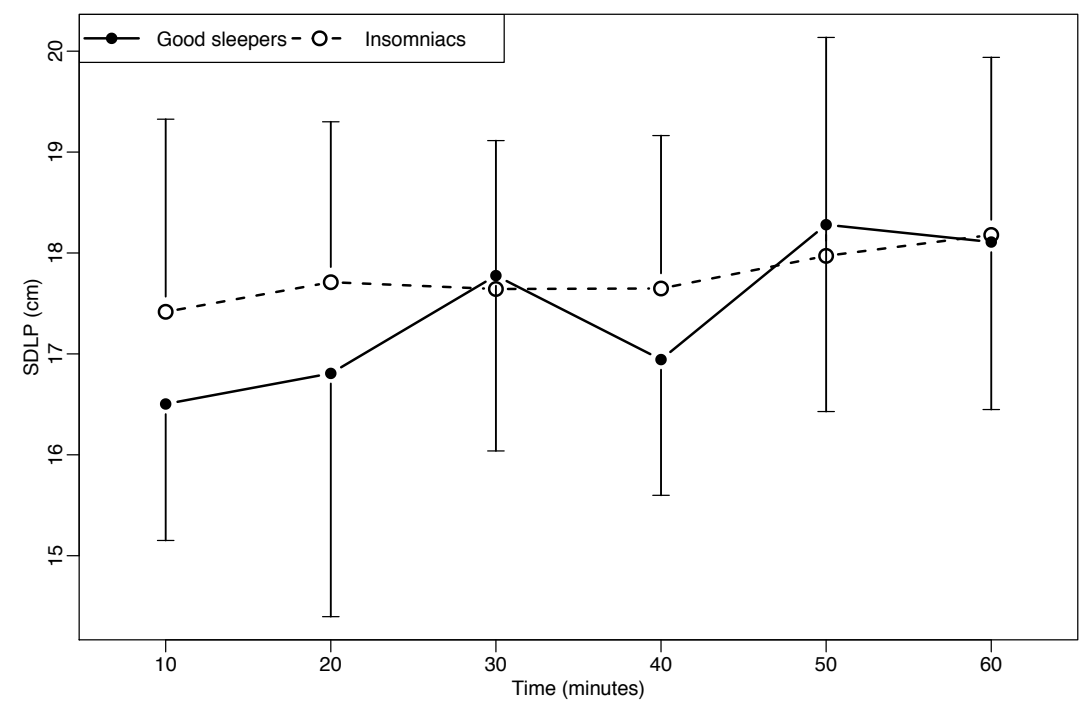

Figure 1. Driving performance of insomnia patients and normal sleepers as measured by Standard Deviation of Lateral Position (SDLP, in $\mathrm{cm}$ ) for each consecutive 10-minute interval of the on-the-road highway driving test (Means $\pm \mathrm{SD}$ ).

Results showed only a significant effect of ToT $\left(p^{\mathrm{LRT}}=0.001\right)$. There was no significant difference between groups, and no significant interaction between Groups and ToT. The model coefficients indicate that SDLP increased with ToT $(p=0.001)$ (See Table 1$)$. 


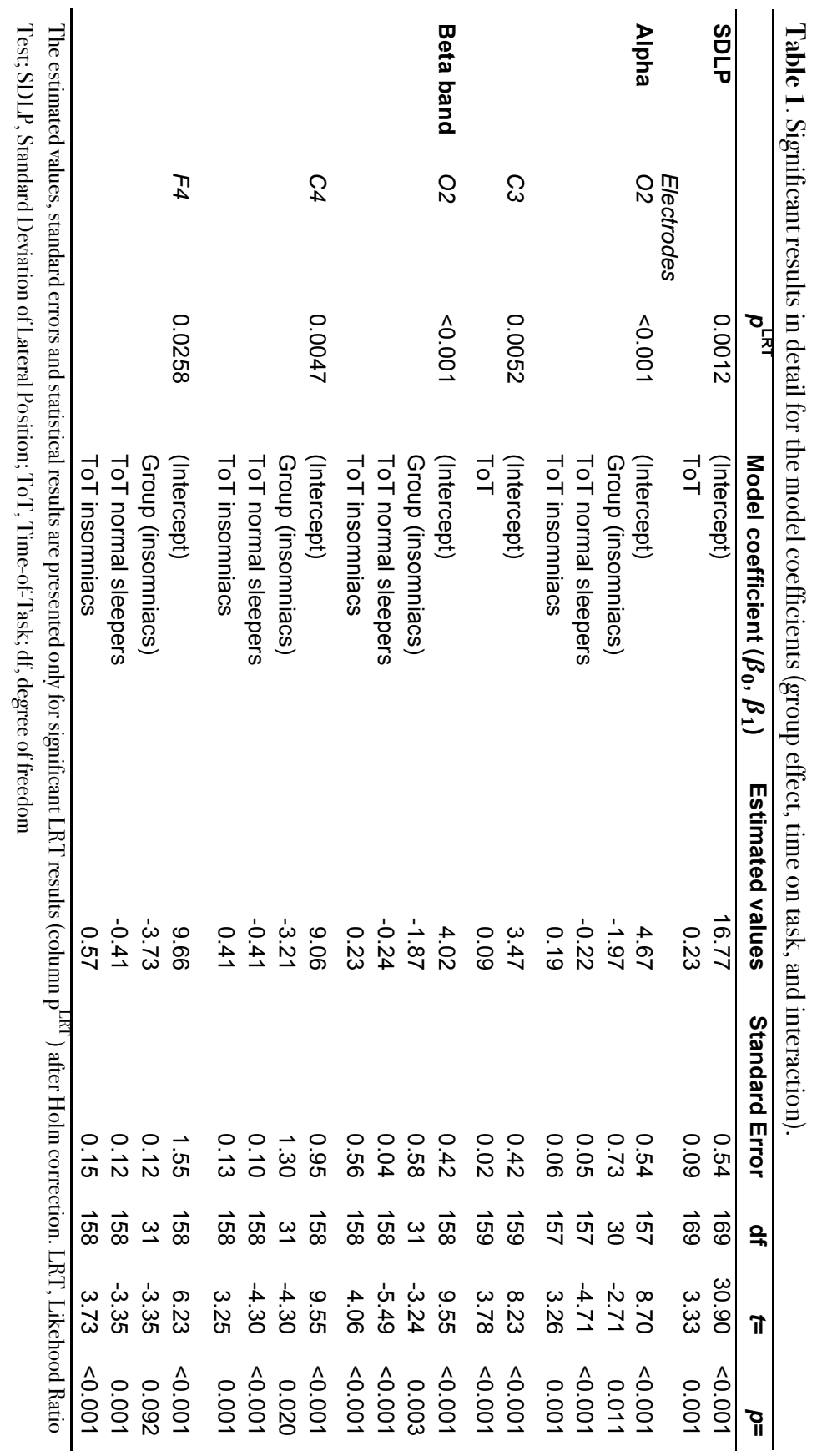




\subsection{Electroencephalography}

Table $1^{1}$ shows details for the best models selected if these contained any significant fixed effects in alpha, theta and beta frequency bands.

In the theta band, no model fitted the data, indicating that there were no significant effects of group and ToT, and no interaction (See Figure 2).

Analysis of the alpha power showed significant effects of group, ToT, and interactions of both factors ( $p^{\mathrm{LRT}}<0.001$, Figure 3$)$. The model coefficients revealed that alpha power at $\mathrm{O} 2$ was lower in insomnia patients than in normal sleepers ( $2.70 \mathrm{vs} 4.67 \mu \mathrm{V}^{2}, p=0.011$ ). In addition, alpha power decreased with ToT in normal sleepers $\left(-0.22 \mu \mathrm{V}^{2} /\right.$ interval, $\left.\mathrm{p}<0.001\right)$, but not in insomnia patients $\left(-0.03 \mu \mathrm{V}^{2} /\right.$ interval, $\left.p<0.001\right)$. There was also a significant effect of the ToT on alpha power at C3 $\left(p^{\mathrm{LRT}}=0.005\right)$. Model coefficients indicate that alpha power increased by $0.085 \mu \mathrm{V}^{2}$ per interval at C3 for both groups $(p<0.001)$.

Analysis of power in beta frequency band showed significant effects of group, ToT, and interactions between both factors (at $\mathrm{O} 2, p^{\mathrm{LRT}}<0.001$; at $\mathrm{C} 4$, $p^{\mathrm{LRT}}=0.005$; at F4, $p^{\mathrm{LRT}}=0.026$ ) (See Figure 4).

Model coefficients indicated that beta power was lower in insomnia patients than in normal sleepers (at $\mathrm{O} 2: 2.15$ vs $4.02 \mu \mathrm{V}^{2}, p=0.003$; at C4: 5.85 vs $9.06 \mu \mathrm{V}^{2}$, $p=0.019$; at $\mathrm{F} 45.93$ vs $9.66 \mu \mathrm{V}^{2}, p=0.092$ ). The effects of ToT differed significantly between insomnia patients and normal sleepers (at $\mathrm{O} 2$, $\mathrm{C} 4$ and $\mathrm{F} 4$, all $p ’ \leq 0.001$ ). Beta power decreased significantly with ToT in normal sleepers (at $\mathrm{O} 2,-0.24 \mu \mathrm{V}^{2}$ per 10 minute interval, $p<0.001$; at $\mathrm{C} 4,-0.41 \mu \mathrm{V}^{2} /$ interval,

71

${ }^{1}$ In the following, we illustrate the interpretation of the model coefficients by means of two examples, the C3 and $\mathrm{O} 2$ electrodes from the alpha band. For C3, the best model supported by LRT $(p=0.0052)$ is a rather simple one without group effect, neither as simple additive effect nor in interaction with the ToT. Therefore, the resulting model corresponds to a linear regression with common intercept $\left(\beta_{0}=3.47\right)$ and slope $\left(\beta_{1}=0.09\right)$ for insomniacs and normal sleepers, both being highly significant $(p<0.001)$. Thus, e.g. the estimated power for both groups for the second interval of length 10 minutes equals $3.47+2 \cdot 0.09$. Group effects are, however, present for $\mathrm{O} 2$ : here, the intercept for the normal sleepers equals $\beta_{0}(g s)=4.02$ $(p<0.001)$, and the intercept of the insomniacs is significantly lower by $1.87(p=0.011)$, resulting in an intercept of $\beta_{0}$ (ins) $=2.15$ for the insomniacs. As to the slope, the result is similar. Normal sleepers have a significant slope of $\beta_{1}(g s)=-0.22(p<0.001)$, while the slope of insomniacs is significantly higher by 0.19 $(p=0.001)$, resulting in a slope coefficient of $\beta_{1}($ ins $)=-0.03$. 
$p=0.001$; at F4, $-0.41 \mu \mathrm{V}^{2} /$ interval, $p=0.001$ ) but not in insomnia patients (at O2, $-0.02 \mu \mathrm{V}^{2} /$ interval, $p=0.001$; at C4, $-0.01 \mu \mathrm{V}^{2} /$ interval, $p=0.001$; at F4, $+0.16 \mu \mathrm{V}^{2} /$ interval, $\left.p<0.003\right)$. Model coefficients are presented in Table 2 .

\subsection{Linear relation between SDLP and EEG measures}

Investigation of a relationship between EEG and SDLP data with a mixed model revealed that none of the EEG measures shows any predictive power for SDLP except for the beta band in the electrode C4. However, this result does not seem relevant as no significant relationship was found for C3 as well as no group difference (See Table 2). 


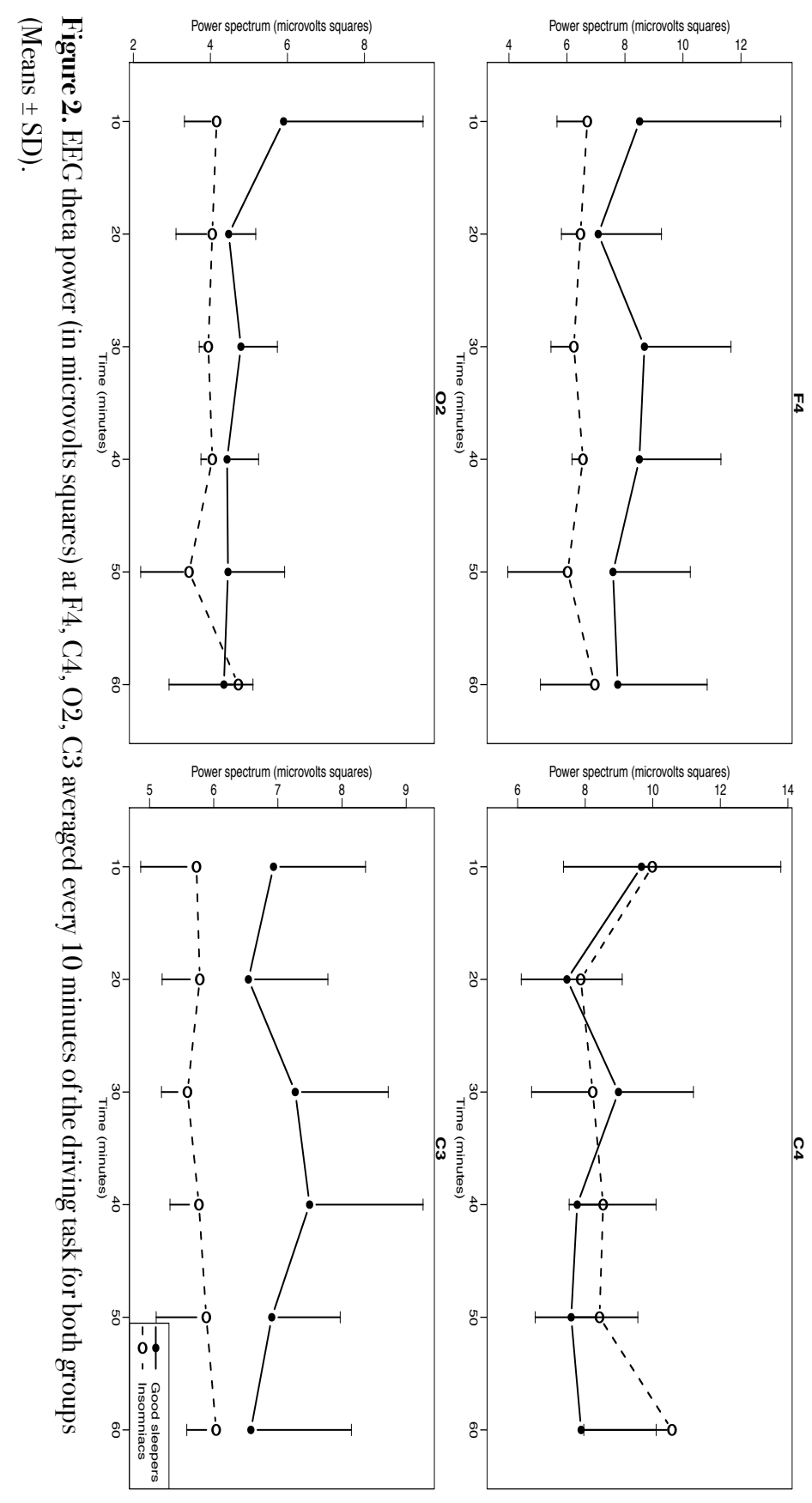


\begin{tabular}{l|l}
74 & Chapter 4
\end{tabular}

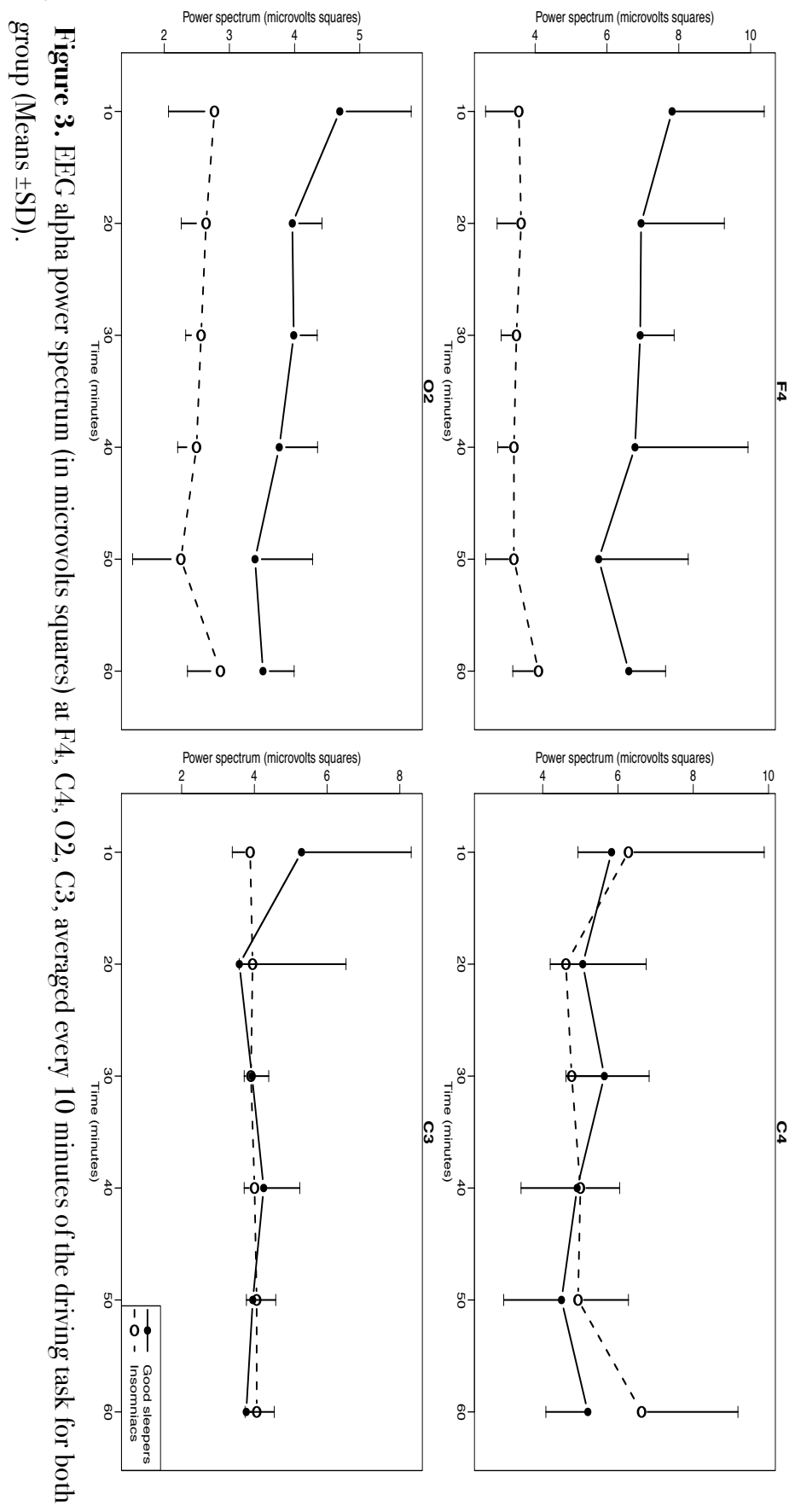




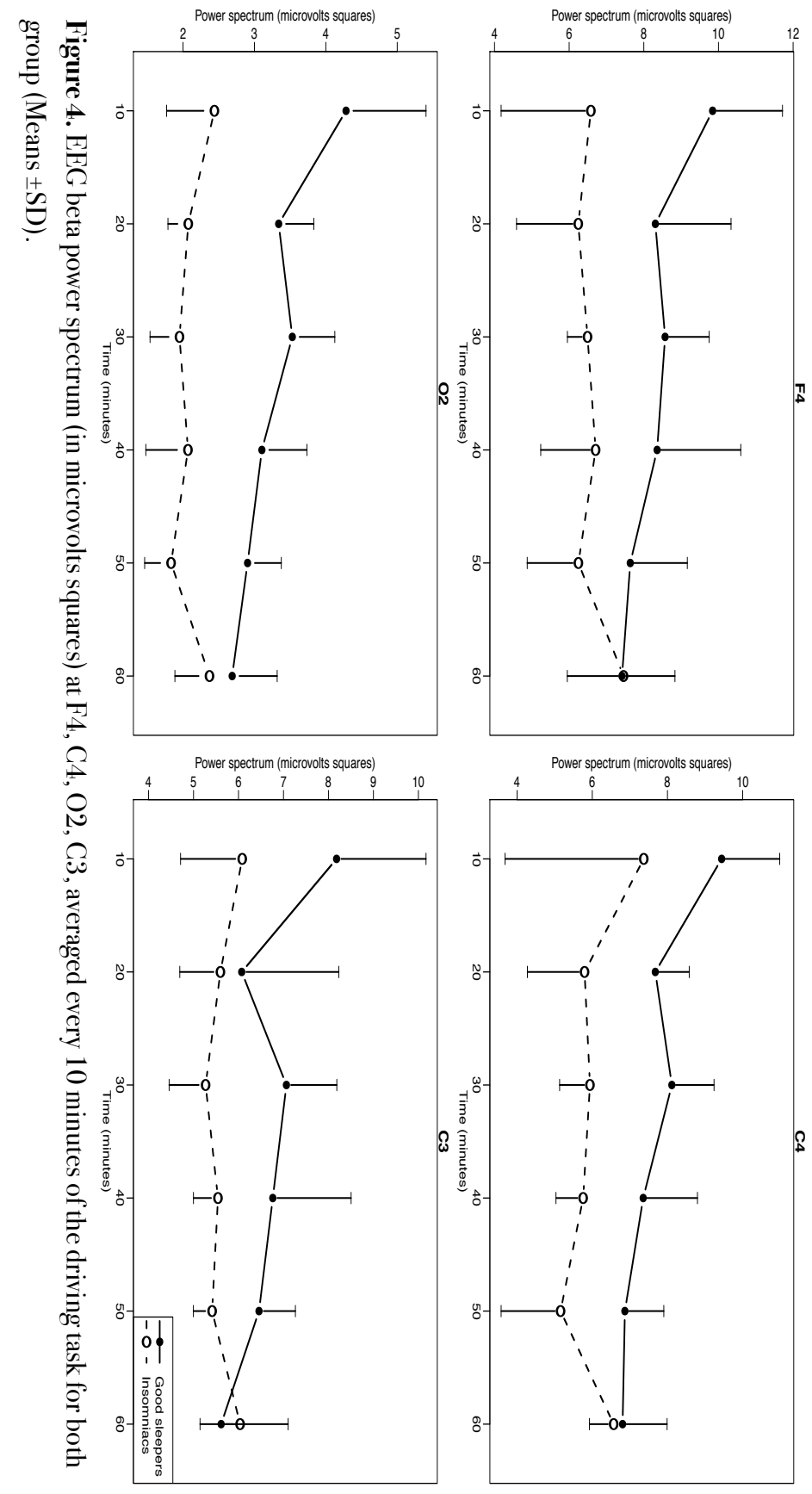




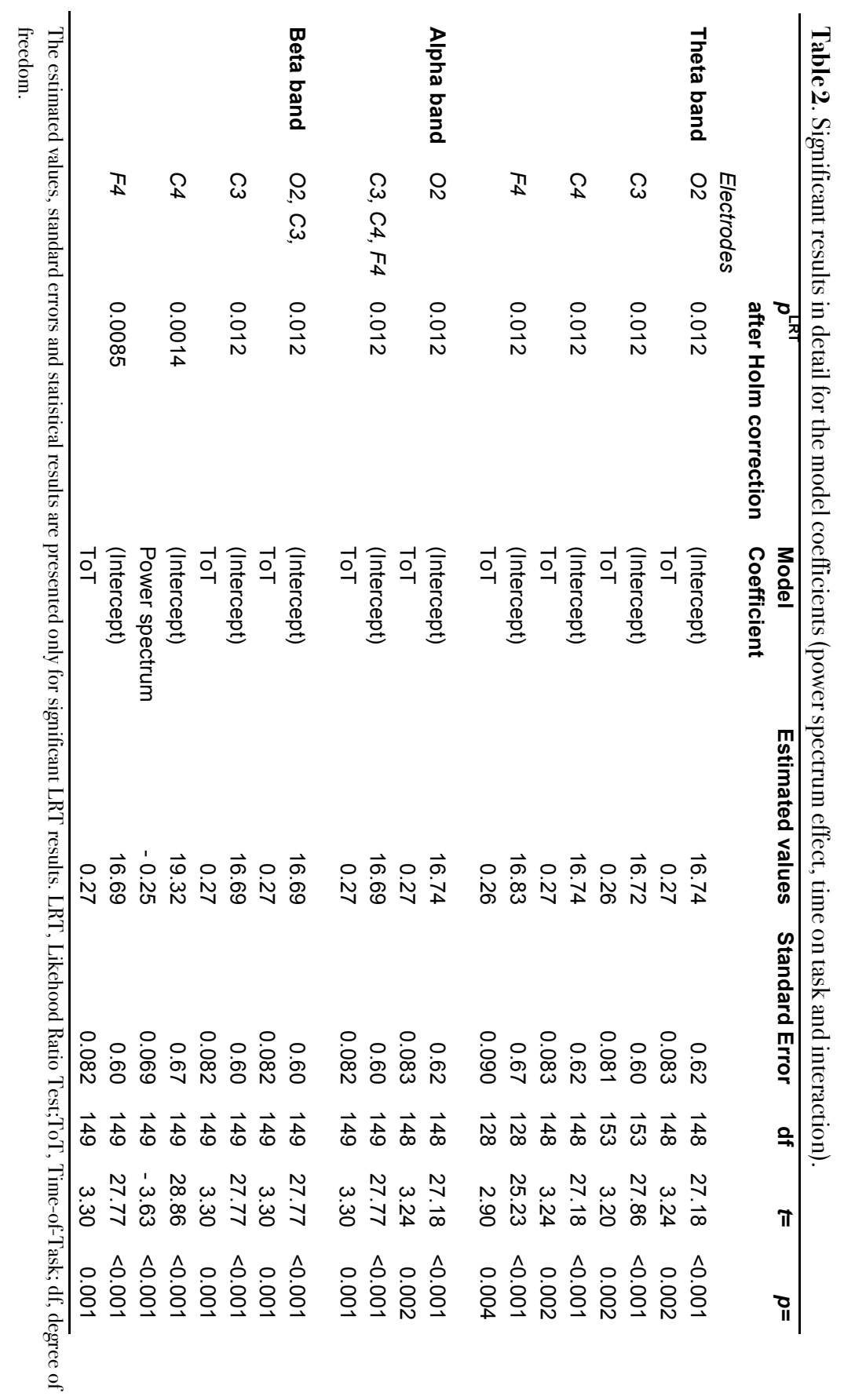




\section{Discussion}

The purpose of the present study was to determine whether changes in driving performance are related to changes in cortical activity during driving in insomnia patients and normal sleepers.

Results show that overall SDLP did not differ between groups, indicating that older untreated insomnia patients were able to drive as good as normal sleepers during an on-the-road highway driving task. There was a significant effect of time on task on SDLP, without a significant interaction, indicating similar performance decrements during driving in both groups. In contrast to SDLP results, analysis of EEG power spectra revealed significant differences between patients and normal sleepers. Overall, insomnia patients displayed less alpha power and less beta power during driving compared to normal sleepers. Furthermore, patients' alpha and beta power remained relatively constant with ToT, whereas the power in both bands decreased with ToT in normal sleepers. Finally, changes in EEG power spectra were not related to changes in SDLP.

The lack of a relationship between SDLP and fluctuations in background EEG is not in line with our expectation. In the past several studies reported a link between EEG power spectra and SDLP in healthy volunteers $(222,231)$, but several other studies did not (232-235). The failure to find a relationship in the present study could be related to the relatively well-rested state of the participants. In their review of the literature on physiological measures of alertness and sustained attention, Oken and colleagues (125) already concluded that the correlation between EEG measures and performance changes on sustained attention tasks, including simulated driving, are "not consistent or large, especially in well-rested participants." Participants in our study were tested in the morning after a normal night of sleep. Most studies trying to identify indices of drowsy driving using EEG, tested healthy young participants after inducing clinically significant levels of drowsiness through sleep restriction, and night-time driving $(52,65,223,224,234,236)$. For example, Johnson and colleagues (235) conducted a study to determine if an individualized EEG-based algorithm could be defined to track performance decrements associated with sleep loss. They concluded that, although the system could effectively track performance 
decrements associated with sleep deprivation, it did not work for well-rested drivers. In addition, participants in our study were older drivers, whereas most studies have used younger volunteers. In a study by Lowden and colleagues (233) comparing simulated night-time driving performance and EEG in young and older drivers, a significant relation between driving performance and brain activity was found in young drivers, but not in older drivers. It is known that young drivers are generally more sensitive to the effects of sleep deprivation, than older drivers (52). The individual variation in physiological parameters may be too large to detect subtle changes during daytime driving in older insomnia patients.

To our knowledge, this is the first study comparing background EEG during driving performance (i.e. eyes open and participants mentally active) between insomnia patients and normal sleepers. Contrary to studies showing elevated beta power in insomniacs during sleep (e.g. (85)), we found that beta power during driving was overall lower in patients than in normal sleepers. However, alpha power was also lower in patients than in normal sleepers. It is not clear, therefore whether patients were more aroused during driving than normal sleepers. Although the lower levels of alpha support the idea that patients were less drowsy during driving, the lower levels of beta power do not support the theory of hyperarousal. It may be possible that daytime waking hyperarousal in insomnia is not reflected in increased beta power, but rather as reduced alpha levels or increases in higher frequencies, such as gamma. However, more work is needed to confirm this assumption.

It is noteworthy that low levels of alpha and beta power in insomnia patients were very stable during the driving test and did not change with ToT. This seems to indicate that EEG power spectra in these patients reflected the underlying state of insomnia rather than any state related changes in arousal or attention that usually arise during a prolonged driving task. The majority of previous studies which investigated EEG measures during driving tasks in nonclinical populations have found slowing of cortical activity with ToT, reflected by relative decreases in beta $(135,226,237,238)$, or increases in alpha and theta power (e.g $(63,115,116,135,227,228,232-234,238-240)$. In line with this we found a decrease in beta power with ToT in the normal sleepers group. SDLP in this group slightly increased as well with ToT, suggesting a vigilance decrement. 
Contrary to previous findings, however, we also found a decrease in alpha power in normal sleepers. Although concurrent reductions in alpha and beta power during monotonous driving have been reported before (237), this pattern of changes does not fit the hypothesis of general slowing of cortical activity with ToT. Therefore it does not support the idea that the overall increase in SDLP over time was due to a decrease in cortical arousal. However, as mentioned above previous driving studies evaluating physiological measures of driver drowsiness differ from the present study by a number of methodological issues, such as driving task (simulators vs real driving), task duration ( 2 to 4 hours of driving vs 1 hour of driving), time of driving (evening and night vs morning), the sleepiness of the participants (sleep deprived vs well rested), the age of the participants (young vs older drivers). These factors all affect the likelihood of measuring significant changes with ToT in physiological markers of alertness and driving performance. As shown in an on-the road study by Verster and colleagues (241) driving at night and for more than two hours had significant impairing effects on performance in a group of healthy young men. Our results showed, however, that on-the-road driving in normal traffic, for only one hour in the morning, after a normal night of sleep resulted only in very small SDLP increase in our sample of older drivers (less than $1 \mathrm{~cm}$ ), despite their complaints of insomnia. The test used in the current study was previously calibrated for the effects of alcohol. The increase in SDLP caused by $0.5 \mathrm{~g} / \mathrm{L}$ alcohol (i.e. $2.4 \mathrm{~cm}$ ) is considered clinically meaningful since accident risk has been demonstrated to increase significantly above this BAC in large epidemiological studies $(242,243)$. Although being insomniac is not strictly comparable to given alcohol doses, this calibration gives an idea about the clinical meaningful our findings. Then, the current investigation did not reveal a SDLP increase with ToT clinically meaningful in older drivers. We may suppose that a longer driving task could have result in larger SDLP increase in both groups. Previous on-the-road driving investigations have revealed that SDLP is an extremely reliable index of individual driving performance with high test-retest reliability (198,244-247). Concerning the relationship between SDLP and crash risk per se, comparative analyses of data obtained from on-the-road driving tests and epidemiological data on crash risks was conducted by Owens and Ramaekers (246). It revealed robust relationships between SDLP increment and indirect risk 
of having a traffic accident in various conditions. These data support the value of SDLP in its relationship to traffic accident risk.

As we did not find any differences between groups performance, older insomnia participants were able to drive as good as older normal sleepers, as measured by SDLP, in a real driving monotonous condition. The present finding differs from those of Perrier and colleagues (144), in which driving performance of primary insomnia patients and normal sleepers was measured by means of a driving simulator. Results of that study revealed that insomnia patients had a larger SDLP than the normal sleepers group and the decrease in driving performance occurred especially after 20 minutes of driving. The discrepancies may be due to the differences in task related factors and participants sample. Patients in the study by Perrier and colleagues (144) were slightly younger (mean age approximately 49 vs 61 years old) and showed significantly worse sleep than normal sleepers the night before testing as measured by polysomnography (sleep efficiency $74 \%$ vs $81 \%$ ) than in the study by Leufkens and colleagues (221). A recent study showed that complaints of daytime impairment were more pronounced in young than in older insomnia patients (212). Moreover impairment is more likely to be associated with more severe insomnia. In addition, Perrier and colleagues used a driving simulator, which has been found to induce more drowsiness and larger ToT effects in monotonous driving tasks than real driving $(53,64,248)$. The increased sensitivity and sleep problems of the younger patients, and the more monotonous nature of the simulated driving task might explain the more pronounced vigilance decrement found in patients as compared to normal sleepers in the study by Perrier and colleagues (144).

It is concluded that daytime on-the-road driving performance of older insomnia patients after a normal night of sleep, as measured by SDLP, does not differ from driving performance of older normal sleepers and that changes in spectral EEG measures of cortical arousal are not related to changes in daytime monotonous highway driving performance in this group of patients. 


\section{Chapter 5 \\ Driving performance and EEG fluctuations during on-the-road driving following sleep deprivation}

Submitted as:

Perrier, J., Jongen S., Vuurman., Bocca, M.-L., Ramaekers, J. G., \& Vermeeren, A. Sleepiness linked to sleep deprivation during on-the-road driving: a behavioral and physiological investigation of time on task effects. 


\begin{abstract}
Sleep deprivation and Time on Task (ToT) can have detrimental effects on vigilance that is the core ability of monotonous driving. However, little is known about the combined effects of sleep deprivation and ToT on real driving performance and cortical activity during driving. The main objective of the present study was to assess whether sleepiness exacerbates ToT effects both on EEG and driving performance in real driving conditions. We also wanted to know which EEG fluctuations are detectable after sleep deprivation during on-the-road driving. Twenty-four healthy participants (25-45 years old) performed a one hour on-the-road monotonous driving task after one night of normal sleep and after one night of sleep deprivation. We measured the Standard Deviation of Lateral Position (SDLP), a measure of road tracking error. Continuous EEG was recorded and spectral power was obtained over three bilateral clusters (frontal, central, parieto-occipital) in the theta $(4-8 \mathrm{~Hz})$, alpha $(8-12 \mathrm{~Hz})$ and beta $(12-30 \mathrm{~Hz})$ bands. Both datasets were averaged every 10 minutes to evaluate ToT effects. Sixteen participants completed the driving task in both sleep conditions (completers) seven drives were terminated prematurely after one night of sleep deprivation (non-completers). We found effects of sleep deprivation, ToT and their interaction both for SDLP and theta and alpha EEG bands. SDLP and EEG indices increased after sleep deprivation and varied with ToT. The latter was more pronounced after sleep deprivation. Beta power spectra increased with ToT only. Compared to completers, the SDLP for non-completers tended to be higher. Beta power spectrum varied differently with ToT between groups in the Frontal cluster. Changes in SDLP and EEG did not correlate significantly. Our study revealed for the first time in on-the-road driving condition that sleep deprivation exacerbates driving impairment and sleepiness as a function of ToT. Our results also suggested that participants who completed both driving sessions put more effort across the task compared to those who prematurely terminated the drives.
\end{abstract}




\section{Introduction}

Vigilance refers to a behavioral level of activity in psychological research while it refers to a state of high cortical activity in psychophysiological research (125) and is a core ability of monotonous driving. Sustained prolonged highway driving highly depends on the abilities of drivers to stay vigilant for longer periods of time especially under monotonous circumstances $(39,40,42,44-47)$. This is supported by simulator $(52,137,234,249,250)$ and on-the-road driving investigations $(65,241,251,252)$ who showed driving performance decrement with Time on Task (ToT) both during day- or nighttime.

In addition to the deleterious effects of ToT, vigilance seems sensitive to the sleep status of participants such as in primary insomnia patients (144) or after sleep deprivation $(34,253)$. A large number of experimental studies have shown the detrimental effects of sleep deprivation on performance (e.g reaction time, number of lapses) during vigilance tasks (35-38). A recent model of attention states that highly automated tasks such as lane keeping during highway driving are more severely affected by sleep deprivation than complex tasks (48). A number of experimental studies have revealed that driving performance decreases during the night or after sleep deprivation both in simulator $(49,50,54,63)$ as well as in onthe-road driving settings $(51,64,65,254)$.

It is well known that ToT effects are exacerbated by sleepiness due to sleep deprivation as revealed by laboratory investigations (59-62). Regarding highway driving, Sagaspe and colleagues (255) investigated whether duration of an on-theroad driving task affects driving performance in the early morning, close to the circadian nadir at $5 \mathrm{am}$. Authors found that after driving for 2,4 or 8 hours, the number of inappropriate lane crossings during the last hour increased with increased duration of prior driving. These results support the notion according to the combined effects of both sleep loss as, time of day and duration of driving, can negatively contribute to driving quality. In contrast, Sandberg and colleagues (65) found limited driving impairment across the task during nighttime driving in comparison to daytime driving probably due to task duration (approximately 30 minutes); driving conditions (i.e rural and urban roads) and time of driving (at 1 and $3 \mathrm{am})$. Nonetheless, it was found that the driving performance was particularly 
affected by ToT effects during nighttime driving in comparison to daytime driving (65). In this study, circadian effects can have interfered with the sleep deprivation effects as the time of driving was before the circadian nadir. Indeed, the vigilance level in influenced by a circadian rhythm process (256) and authors investigated the driving performance of participants when the vigilance circadian rhythm is low. As authors did not compare directly one night of sleep deprivation with a normal night of sleep; it is not clear whether the results reported in the study from Sandberg and colleagues were directly linked to sleep deprivation or if circadian effects were also present. Therefore, little is known about the combined effects of sleep deprivation and ToT on actual driving performance.

Epidemiological studies support the idea according to sleep deprivation and ToT can have combined effects on driving performance. Indeed, it has been reported that the crash risk (i.e lane crossings) following sleep deprivation seems most pronounced during extended driving periods when loss of vigilance is highest (26-28). The development of drowsy drivers' detection systems should thus give benefit in the management of fatigue and sleepiness risk during driving. These types of devices are based, among others, on physiological and neurobehavioral indicators (257). Such indicators can easily be obtained by using EEG fluctuations as important sources of information on sleepiness and vigilance states of the driver (236). Until now, there are no current regulatory guidelines regarding the appropriate use of these drowsy drivers' detection systems.

Several experimental studies have reported EEG fluctuations during vigilance tasks (114) and more specially during driving experiments (125). The EEG results are combined in different frequency bands. Low frequency delta and theta brain waves (1-8 Hz) are considered objectives markers of sleepiness $(30,136,258,259)$. Alpha waves $(8-12 \mathrm{~Hz})$ are related to sleepiness and fatigue $(115,228)$. It has also been reported that alpha is sensitive to attentional demands $(120,130,260)$. Beta waves $(12-30 \mathrm{~Hz})$ are related to regulation of vigilance and arousal $(131,132)$ and increased effort to maintain adequate performance under sleep deprivation condition (134).

Laboratory investigations revealed that sleep deprivation is associated with an increase of theta and alpha power at rest (136) as well as during task performance $(36,112,261)$. Results from sleep deprivation or night driving 
studies showed increased levels of alpha and theta waves both in simulator $(49,50,52,254)$ and on-the-road settings $(254)$. In addition, a previous on-theroad driving investigation found increased Karolinska Drowsiness Score (KDS), which is a combined measure of EEG alpha and theta activity and slow eye movements, in condition of night-time driving compared to day-time driving (65) (Sandberg 2011). The two EEG investigations conducting during real driving cited above have compared nighttime and daytime driving $(65,254)$ without evaluating the direct effect of a night of sleep deprivation to a normal night of sleep. Commonly, during sustained attention tasks, ToT induces an increase in the theta power $(111,115,137)$ as well as an increase in alpha power $(262-264)$. Some studies have also reported larger beta power as ToT increases $(115,133)$, suggested to reflect compensatory effort to maintain levels of performance as vigilance and arousal decreased. During monotonous driving in both simulator and on-the-road settings, ToT has been found to lead to an increase in alpha and theta EEG power spectra $(63,116,222,232,249)$. Thus, little is known about the separated and combined effects of sleep deprivation and ToT on EEG activity during on-the-road driving.

In addition, the relationship between on-the-road driving performance and EEG power spectra following one night of sleep deprivation is not clear. Indeed, previous driving studies found discrepant results rirrespective of the setting used (i.e simulator or on-the-road driving). Both driving performances and EEG indices have been found to be correlated by some studies, $(222,265)$, while several other studies failed to find any relationship between these indices (232$235)$.

The main objective of the present study was to assess whether sleepiness exacerbates ToT effects both on EEG and driving performance in real driving conditions. We also wanted to know which EEG fluctuations are detectable after sleep deprivation during on-the-road driving. We predict increased SDLP both after sleep deprivation and across ToT. We expect that beta, theta and alpha increase both after sleep deprivation and with ToT. Also, interaction effects of sleep deprivation and ToT are expected on driving performance decrement and on the increase of theta and alpha. 


\section{Methods}

\subsection{Participants}

Twenty-four healthy male and female volunteers (ages 23-45 years) were recruited through poster advertisements at Maastricht University. Initial screening was based on a medical history questionnaire examined by a medical supervisor. Subjects underwent a physical examination, which included urinalysis, tests for drugs of abuse (amphetamines, benzo- diazepines, cannabis, cocaine, 3,4methylenedioxymethamphetamine, and opiates), and a 12-lead electrocardiogram. For participation, the following inclusion criteria had to be met: possession of a valid driving license for four years or more, driving experience of at least $5000 \mathrm{~km}$ per year on average and a body mass index (BMI) between 19 and $29 \mathrm{~kg} \mathrm{~m}^{-2}$, good sleep quality, i.e. score $<7$ on the Pittsburg Sleep Quality Index (266). Exclusion criteria included: shift workers, history of a sleep disorder, extreme morning or evening types as assessed by the Morning-Evening Questionnaire, with scores < 30 or $>70$ (148); any history of psychiatric or medical illness; history or current drug or alcohol abuse; current use of psycho-active medication; and inability to stay abstinent during the study from any drug or alcohol abuse; excessive caffeine use, defined as drinking six or more cups of coffee per day; and smoking.

A total of 24 participants (12 men, 12 women) completed the study. Their mean $( \pm \mathrm{SD})$ age was $26.9( \pm 3.4)$ years. The study was conducted in accordance with the code of ethics on human experimentation established by the declaration of Helsinki (1964) and amended in Seoul (2008). All participants were informed of the study's goal, procedures, and potential hazards in writing, and they indicated their informed consent in writing. The Medical Ethics Committees of Maastricht University approved the study. Participants received a financial compensation for their participation in the study.

\subsection{Design}

The study was conducted according to a two-period cross-over design to compare the effects of one night of sleep deprivation and a normal night of sleep on driving performance and EEG. Test days were separated by at least one week, and the order of the two conditions was balanced over participants. 
This paper presents data from a larger study aimed to investigate the validity and sensitivity of psychometrics tests for measuring driving impairment (267). The present paper will only discuss effects of ToT and sleep deprivation on EEG during the on-the-road driving task.

\subsection{Procedure}

Participants were individually trained to perform the driving test prior to the first test day. During participation in the study, alcohol intake was not allowed from $24 \mathrm{~h}$ prior to each test day until discharge. On treatment days, caffeine intake and smoking was not allowed until discharge. Participants agreed not to use any drugs of abuse or oral medication (except oral contraceptives and aspirin) during the study. Participants yielded urine and breathe samples to confirm their compliance with prohibitions against use of drugs and to verify a BAC of $0.0 \mathrm{~g} /$ at the beginning of each session. To verify good sleep quality the night before testing, the Groningen Sleep Quality (268) was administered; participants with a score $>7$ were dismissed and rescheduled. Both testing days ended at 13:00 pm after removing EEG caps. After normal sleep, participants were dismissed; after sleep deprivation, participants were driven home.

\subsection{Assessment}

On-the-road highway driving test

In the standardized highway driving test (108), the participant operates a specially instrumented vehicle over a 100-km (61-mile) primary highway circuit, accompanied by a licensed driving instructor having access to dual controls. The task of the participant is to maintain a constant speed of $95 \mathrm{~km} \mathrm{h-1} \mathrm{(58} \mathrm{miles} \mathrm{h-1)}$ and a steady lateral position between the delineated boundaries of the right traffic lane. The participant is only allowed to deviate from this instruction to pass slower vehicles in the same lane and to execute the reversal of direction at the mid-test turn around. The role of the driving instructor is only to ensure safety of the test. Participants are instructed beforehand to terminate the test by stopping the vehicle on the road shoulder if they have doubts about their competence to continue safely. If the driving instructor judges a participant's performance as becoming unsafe he can also instruct the participant to stop the vehicle. 
The vehicle speed and lateral position are recorded continuously. These signals are edited off-line to remove data recorded during overtaking maneuvers or disturbances caused by roadway or traffic situations. The primary outcome variable is the standard deviation of lateral position (SDLP, in $\mathrm{cm}$ ) that was calculated for successive $5 \mathrm{~km}$ segments and over the test as a whole. SDLP is a measure of road tracking error, or 'weaving'. The test duration was approximately one hour with one turn around after approximately 30 minutes of driving. To analyze Time-on-Task effects the SDLP scores of segments 2 to 18 were averaged per test by six subdivisions of nearly 10 minutes (S1, S2, S3, S4, S5 and S6).

Electroencephalography recording and preprocessing

For the assessment of brain activity while driving, electroencephalography (EEG) was recorded by the system of Brain Products (see http://www.brainproducts.com/). It consists of a portable amplifier, $32 \mathrm{Ag}$-AgCl active electrodes (actiCAP) and the recording software (Brain Vision Recorder) Active electrodes were referenced online to an electrode over the left mastoid. An average mastoid reference was computed offline and subtracted from all scalp channels. To obtain bipolar vertical EOG recordings, electrodes were attached vertically above below the left eye. Electrodes impedance was brought to less than $10 \mathrm{k} \Omega$ before data collection commenced. EEG and EOG data were recorded from 0 to $70 \mathrm{~Hz}$ and sampled online at $250 \mathrm{~Hz}$. Offline analysis of the EEG data was performed as follows. EEG data were bandpass filtered between 0.1 and 30 $\mathrm{Hz}$, and segmented into 50\%-overlapping $7.5 \mathrm{~s}$ epochs within each period of interest. EEGs were corrected for vertical and horizontal eye movements based on the method of Gratton and Coles (269) and epochs with voltage changes larger than $200 \mu \mathrm{V}$ across the recording window were rejected. Power Spectral Analysis (PSA) is performed by computing Fast Fourier Transforms (FFT), and power spectra were computed from 1 to $30 \mathrm{~Hz}$. Spectral power was calculated in the three major EEG bands: theta (4-8 Hz), alpha (8-12 Hz) and beta $(12-30 \mathrm{~Hz})$, and average power in these bands was obtained over three bilateral clusters as follows: frontal cluster (Fp1,Fp2, F3, F4, Fz, F8, F7); Central cluster (FC3, FC4, C3, C4, Cz, CP3, CP4) and Parieto-occipital cluster (P3, P4, Pz, P7, P8, O1, $\mathrm{O} 2)$. As we recorded EEG during and on-the-road driving task, delta activity was 
not analyzed because of the number of artifacts generated by body movements of the driver in the car. Data were analyzed using BrainVision (version 2.0).

\subsection{Statistical analysis}

Eight out of 24 subjects did not complete the driving test as scheduled due to extreme drowsiness in the sleep deprivation condition. Of those, one withdrew already before the start of the driving test. So, a total of 16 participants completed both driving tests as scheduled. These groups will here-after be referred to as completers $(n=16)$ and non-completers $(n=7)$.

A first analysis included data from the completers only. All driving and EEG parameters for this sample were analyzed using a 2x6 repeated measures ANOVA with Sessions (two levels; sleep deprivation versus normal sleep) and ToT (six levels; S1 to S6) as within subject factors. To determine whether there was a difference between completers and non-completers, a second analysis was done over data recorded during the first 30 minutes of driving. This analysis used a $2 \times 2 \times 3$ repeated measures ANOVA with Groups (two levels; completers vs noncompleters) and ToT (three levels; S1 to S3) as within subject factors. All ANOVA's were done for three separate bilateral clusters of electrodes (frontal, central, parieto-occipital).

Correlations between changes from the normal sleep condition to the sleep deprivation condition in means SDLP and means EEG (averages obtained on the $30 \mathrm{~min}$ ) were obtained. In addition, we quantified correlation between SDLP and EEG power spectra as changes over time after normal sleep (S1 versus S6) and after sleep deprivation (S1 versus S3). All these results were made including all participants for each EEG band (theta, alpha, beta) and for each cluster of electrodes (frontal, central, parieto-occipital) were made using the Spearman coefficient correlation. The three factors ANOVA were performed with Statistica version 10, the rest of the statistical analyses were done using Sigmastat version 3.5 . 


\section{Results}

\subsection{Missing data and prematurely terminated driving tests}

One participant withdrew from further testing during the sleep deprivation condition after 20 hours of wakefulness because he felt unable to continue due to excessive sleepiness. Due to technical problems with EEG material during the driving test, no EEG data were obtained for two participants for the sleep deprivation condition.

Seven driving tests were terminated before scheduled completion, all in the sleep deprivation condition. For one of them, the driving instructor judged the participant to be too drowsy to continue safely and took the decision to stop. For the six other ones, participants felt too drowsy to continue safely and took the decision to stop themselves. All premature terminations occurred between 30 and 60 minutes of the driving test. The mean duration $( \pm \mathrm{SD})$ of the total driving period for non-completers in the sleep deprivation condition was $36 \pm 10$ minutes.

\subsection{Driving performance and EEG fluctuations in completers}

On-the-road highway driving test

Figure 1a shows means $( \pm$ SEM) of SDLP by ToT for completers after normal sleep and sleep deprivation. The 2x6 repeated measures ANOVA showed significant main effects of Session $\left(\mathrm{F}_{1,15}=22.98, p<0.001\right)$, ToT $\left(\mathrm{F}_{5,75}=9.70, p<0.001\right)$ and an interaction between these factors $\left(\mathrm{F}_{5,73}=3.27, p=0.010\right)$. SDLP was overall higher after sleep deprivation as compared to normal sleep. Figure 1a shows that SDLP increased with ToT, but more so after sleep deprivation than after normal sleep.

\section{Electroencephalography data}

The results of the $2 \times 6$ repeated measures ANOVA on EEG data for completers are reported in the Table 1 . Results show main effects of Session, ToT and interactions between these two factors both for the theta and alpha power spectra. For the beta power spectrum, a ToT effect and a trend for Session by ToT interaction was found in the Central cluster. 
The main effect of Session indicates that alpha and theta power spectra were significantly increased in the sleep deprivation condition as compared to the normal sleep condition at all three clusters of electrodes. In contrast, beta power spectrum did not differ significantly between normal sleep and sleep deprivation conditions.

The main effect of ToT indicates that theta, alpha and beta power spectra differed between subdivisions. The significant interaction indicates that these changes differed for both sessions. Figure $1 \mathrm{~b}$ shows that after normal sleep the theta power spectrum at S3 and S4 higher compared to other subdivisions. These differences seemed enhanced after sleep deprivation. Figure 1c shows that alpha power spectrum is relatively constant with ToT after normal sleep. After sleep deprivation alpha power increases, in particular in S3 and S4. Figure 1d shows that beta power spectrum generally increased from S1 to S6 for all clusters of electrodes. Beta power after sleep deprivation did not differ significantly from that after normal sleep. Although we found only a trend for the Session by ToT interaction of the beta power spectrum at the Central cluster, Figure 1d indicates that the changes over time are more pronounced more pronounced in the sleep deprivation condition than in the normal sleep condition, especially at the Central cluster. 
Table1. Electroencephalography results - Completers.

\begin{tabular}{|c|c|c|c|c|c|c|c|}
\hline & & \multicolumn{2}{|c|}{ Theta } & \multicolumn{2}{|c|}{ Alpha } & \multicolumn{2}{|c|}{ Beta } \\
\hline & & $\mathrm{F}$ & $p$ & $\mathrm{~F}$ & $p$ & $\mathrm{~F}$ & $p$ \\
\hline \multirow[t]{3}{*}{ Frontal } & Sessions & 7.28 & $0.018^{* *}$ & 5.90 & $0.030^{* *}$ & 0.022 & 0.891 \\
\hline & ToT & 17.82 & $<0.001^{* * *}$ & 7.61 & $<0.001^{\star * *}$ & 3.82 & $0.004^{* *}$ \\
\hline & $\begin{array}{l}\text { Sessions } \\
\mathrm{X} \text { ToT }\end{array}$ & 3.60 & $0.006^{* *}$ & 2.96 & $0.018^{* *}$ & 0.33 & 0.902 \\
\hline \multirow[t]{3}{*}{ Central } & Sessions & 7.31 & $0.018^{* *}$ & 7.36 & $0.018^{\star *}$ & 0.027 & 0.870 \\
\hline & ToT & 13.98 & $<0.001^{* * *}$ & 5.52 & $<0.001^{* * *}$ & 5.09 & $<0.001^{* * *}$ \\
\hline & $\begin{array}{l}\text { Sessions } \\
\mathrm{X} \text { ToT }\end{array}$ & 3.02 & $0.016^{* *}$ & 3.30 & $0.010^{\star *}$ & 2.15 & 0.071 \\
\hline \multirow{3}{*}{$\begin{array}{l}\text { Parieto- } \\
\text { occipital }\end{array}$} & Sessions & 4.77 & $0.048^{* *}$ & 5.61 & $0.034^{\star *}$ & 0.0004 & 0.980 \\
\hline & ToT & 14.26 & $<0.001^{* * *}$ & 6.21 & $<0.001^{* * *}$ & 3.36 & $0.009^{* *}$ \\
\hline & $\begin{array}{l}\text { Sessions } \\
\text { X ToT }\end{array}$ & 2.16 & 0.069 & 2.09 & 0.079 & 1.64 & 0.156 \\
\hline
\end{tabular}

Data were analyzed for 60 minutes of driving in the 16 participants who completed the driving task. F and $p$ values are reported for the two-factors repeated measures ANOVA with Sessions (two levels; Sleep deprivation vs normal sleep) and Time-on-Task (six levels corresponding to six blocks of 10 minutes named as Subdivisions) as within subject factors. df are respectively $\mathrm{F}_{(1,13)}$; and $\mathrm{F}_{(5,65)}$. $p<0.05$ was considered as significant. ${ }^{* *} p<0.05,{ }^{* * *} p<0.001$. ToT, Time-on-Task. 

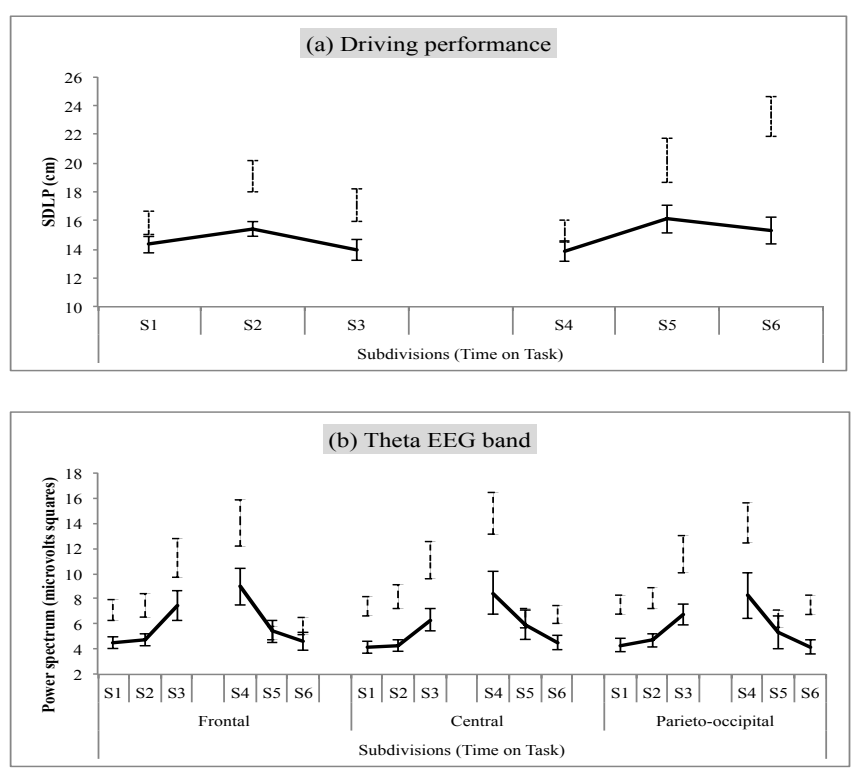

(c) Alpha EEG band

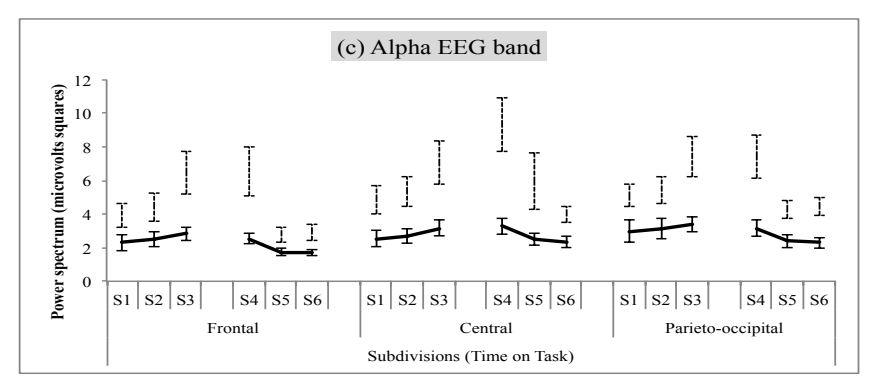

(d) Beta EEG band

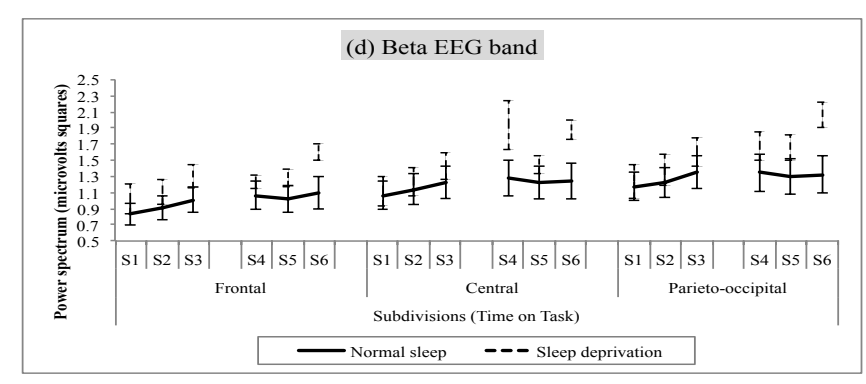

Figure 1. Driving performance and EEG fluctuations represented by the mean \pm SEM across Time on Task (Subdivisions) for both conditions (Normal sleep and Sleep deprivation). Continuous lines: normal sleep; dashed lines: sleep deprivation. Data are presented for completers only $(n=16)$. SDLP, Standard Deviation of Lateral Position; EEG, electroencephalography. 


\subsection{Comparisons between completers and non-completers}

On-the-road highway driving test

Figure 2a shows means $( \pm$ SEM) of SDLP over the first 30 minutes of driving for completers and non-completers after normal sleep and sleep deprivation. The $2 \times 3 \times 2$ mixed model ANOVA showed significant or nearly significant main effects of Group $\left(\mathrm{F}_{1,21}=3.79, p=0.065\right)$, Session $\left(\mathrm{F}_{1,21}=25.03, p<0.001\right)$ and ToT $\left(\mathrm{F}_{2,42}=8.73, p<0.001\right)$. SDLP tended to be higher for non-completers than for completers and was significantly increased after sleep deprivation in comparison with normal sleep condition. SDLP varied significantly with ToT. No significant interactions were found, indicating that effects of sleep deprivation and ToT did not differ between groups. 
Table 2. Electroencephalography results - Completers vs on-completers.

\begin{tabular}{|c|c|c|c|c|c|c|c|}
\hline & & \multicolumn{2}{|c|}{ Theta } & \multicolumn{2}{|c|}{ Alpha } & \multicolumn{2}{|c|}{ Beta } \\
\hline & & $F$ & $p$ & $\mathrm{~F}$ & $p$ & $F$ & $p$ \\
\hline \multirow[t]{5}{*}{ Frontal } & Groups & 0.58 & 0.456 & 0.98 & 0.335 & 0.015 & 0.904 \\
\hline & Sessions & 4.90 & $0.039^{* *}$ & 9.94 & $0.005^{\star *}$ & 0.019 & 0.891 \\
\hline & ToT & 20.43 & $<0.001^{* * *}$ & 22.05 & $<0.001^{* * *}$ & 16.67 & $<0.001^{* * *}$ \\
\hline & $\begin{array}{l}\text { Sessions } \\
\text { X ToT }\end{array}$ & 1.44 & 0.249 & 3.14 & 0.055 & 4.41 & $0.019^{* *}$ \\
\hline & Groups X & 0.21 & 0.811 & 2.23 & 0.122 & 4.33 & $0.020^{* *}$ \\
\hline \multirow[t]{5}{*}{ Central } & Groups & 0.93 & 0.345 & 0.55 & 0.467 & 0.31 & 0.585 \\
\hline & Sessions & 8.36 & $0.009^{* *}$ & 11.07 & $0.004^{* *}$ & 0.018 & 0.893 \\
\hline & ToT & 15.72 & $<0.001^{* * *}$ & 12.5 & $<0.001^{* * *}$ & 19.98 & $0.001^{* * *}$ \\
\hline & $\begin{array}{l}\text { Sessions } \\
\mathrm{X} \text { ToT }\end{array}$ & 0.64 & 0.533 & 0.80 & 0.456 & 4.42 & $0.019^{* *}$ \\
\hline & $\begin{array}{l}\text { Groups X } \\
\text { ToT }\end{array}$ & 0.27 & 0.766 & 1.85 & 0.171 & 1.55 & 0.225 \\
\hline \multirow{5}{*}{$\begin{array}{l}\text { Parieto- } \\
\text { occipital }\end{array}$} & Groups & 1.31 & 0.266 & 0.85 & 0.367 & 0.35 & 0.562 \\
\hline & Sessions & 7.18 & 0.144 & 11.11 & $0.003^{* *}$ & 0.14 & 0.713 \\
\hline & ToT & 20.19 & $<0.001^{* * *}$ & 13.94 & $<0.001^{* * *}$ & 16.94 & $<0.001^{\star * *}$ \\
\hline & $\begin{array}{l}\text { Sessions } \\
\text { X ToT }\end{array}$ & 0.77 & 0.472 & 0.48 & 0.621 & 4.72 & $0.015^{\star * *}$ \\
\hline & $\begin{array}{l}\text { Groups X } \\
\text { ToT }\end{array}$ & 0.75 & 0.478 & 2.89 & 0.068 & 2.02 & 0.147 \\
\hline
\end{tabular}

Data were analyzed for 30 minutes of driving in all the participants. F and p values are reported for the threefactors repeated measures ANOVA with Groups (completed vs non-completers), Sessions (two levels; sleep deprivation vs normal sleep) and Time-on-Task (three levels corresponding to the first three blocks of 10 minutes of the driving task named as Subdivisions) as within subject factors. $\mathrm{df}$ are respectively $\mathrm{F}_{(1.19)}$; and $\mathrm{F}_{(2,38) \cdot} \cdot p<0.05$ was considered as significant. ${ }^{* *} p<0.05,{ }^{* * *} p<0.001$. ToT, Time-on-Task. 

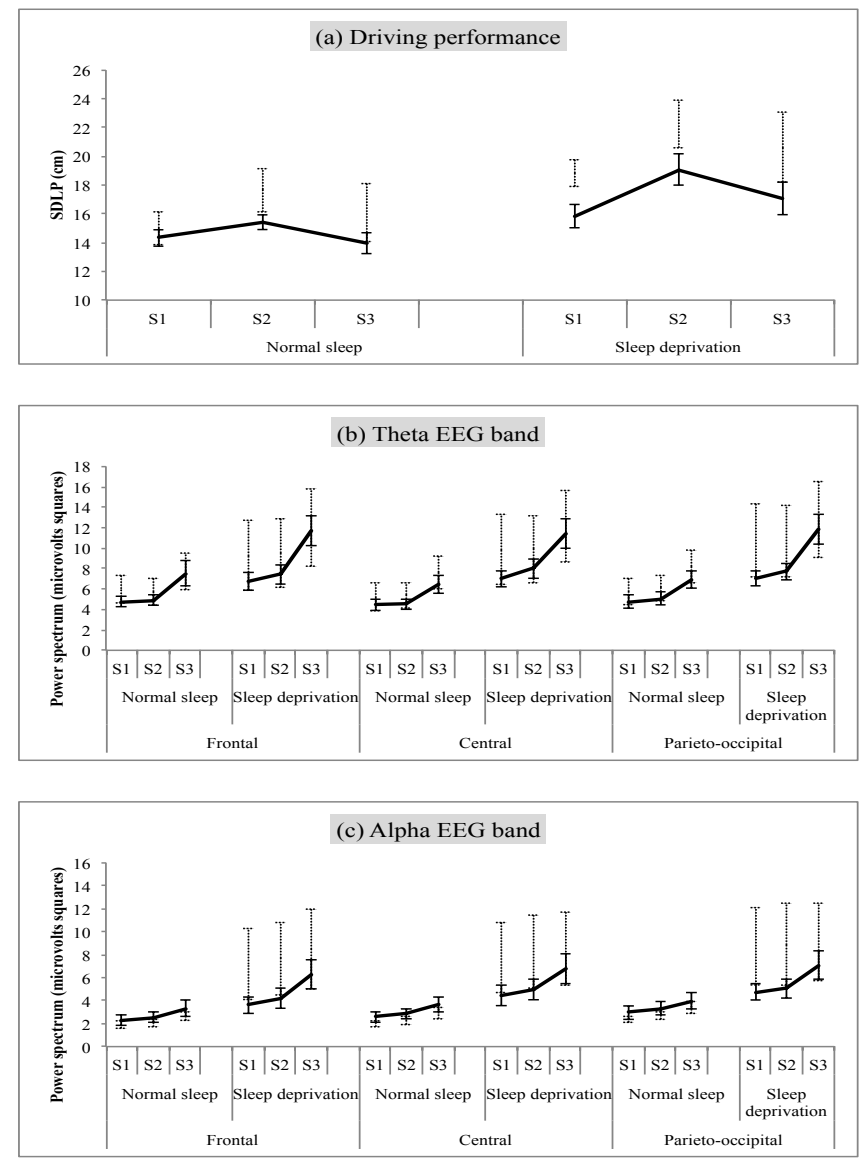

(d) Beta EEG band

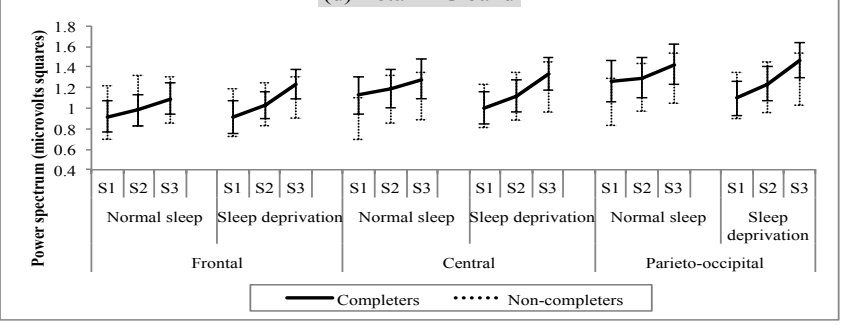

Figure 2. Driving performance and EEG fluctuations represented by the mean \pm SEM across Time on Task (Subdivisions) for both conditions (Normal sleep and Sleep deprivation). Data are presented for both groups; completers and non-completers $(\mathrm{n}=16$ and 7 , respectively).

Continuous lines: completers participants; dashed lines: noncompleters participants. SDLP, Standard Deviation of Lateral Position; EEG, electroencephalography.

\section{Electroencephalography data}


Figures $2 \mathrm{~b}, \mathrm{c}, \mathrm{d}$ show means $( \pm \mathrm{SEM}$ ) of SDLP over the first 30 minutes of driving for completers and non-completers after normal sleep and sleep deprivation. The results of the $2 \times 3 \times 2$ mixed model ANOVA on EEG data for completers and noncompleters during the first 30 minutes of driving are reported in the Table 2. Results showed no significant differences between groups for all frequency bands. No significant effect for interaction was found both for theta and alpha power spectra (See Figure 2c). Only beta power showed a significant Group by ToT interaction in the Frontal cluster. Figure 3 shows that, for both sessions pooled together, the beta power spectrum varied more across the task for completers than for non-completers.

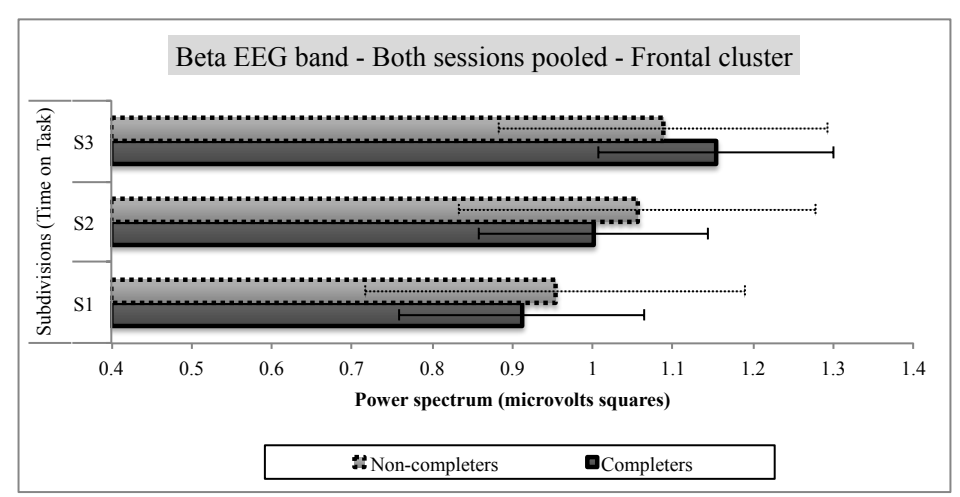

Figure 3. EEG beta power fluctuations represented by the mean \pm SEM across Time on Task (Subdivisions) for both conditions pooled together (Normal sleep and Sleep deprivation) at the Frontal cluster. Data are presented for both groups; completers and noncompleters ( $\mathrm{n}=16$ and 7, respectively). Dark background fill: completers; grey background fill: noncompleters. EEG, electroencephalography. 


\subsection{Correlation between SDLP and EEG power spectra}

The Table 3 reports the main results of the correlations between SDLP and background EEG parameters. None of the correlation was significant.

Table 3. Correlations between SDLP and EEG measures.

\begin{tabular}{|c|c|c|c|c|}
\hline & & Theta & Alpha & Beta \\
\hline \multirow[t]{3}{*}{ Frontal } & $\begin{array}{l}\text { Changes from NS to } \\
S D-30 \text { minutes }\end{array}$ & $\begin{array}{l}r=0.167 \\
p=0.473\end{array}$ & $\begin{array}{l}r=0.194 \\
p=0.395\end{array}$ & $\begin{array}{l}r=0.140 \\
p=0.551\end{array}$ \\
\hline & $\begin{array}{l}\text { Changes with ToT - } \\
\text { NS }\end{array}$ & $\begin{array}{l}r=-0.225 \\
p=0.286\end{array}$ & $\begin{array}{l}r=0.109 \\
p=0.609\end{array}$ & $\begin{array}{l}r=-0.098 \\
p=0.644\end{array}$ \\
\hline & Changes with ToT -SD & $\begin{array}{l}r=0.112 \\
p=0.621\end{array}$ & $\begin{array}{l}r=0.220 \\
p=0.331\end{array}$ & $\begin{array}{l}r=-0.109 \\
p=0.639\end{array}$ \\
\hline \multirow[t]{3}{*}{ Central } & $\begin{array}{l}\text { Changes from NS to } \\
S D-30 \text { minutes }\end{array}$ & $\begin{array}{l}r=0.296 \\
p=0.129\end{array}$ & $\begin{array}{l}r=0.256 \\
p=0.258\end{array}$ & $\begin{array}{l}r=0.177 \\
p=0.448\end{array}$ \\
\hline & $\begin{array}{l}\text { Changes with ToT - } \\
\text { NS }\end{array}$ & $\begin{array}{l}r=0.054 \\
p=0.799\end{array}$ & $\begin{array}{l}r=0.037 \\
p=0.860\end{array}$ & $\begin{array}{l}r=-0.076 \\
p=0.722\end{array}$ \\
\hline & Changes with ToT -SD & $\begin{array}{l}r=0.071 \\
p=0.754\end{array}$ & $\begin{array}{l}r=0.207 \\
p=0.361\end{array}$ & $\begin{array}{l}r=-0.040 \\
p=0.861\end{array}$ \\
\hline \multirow[t]{3}{*}{$\begin{array}{l}\text { Parieto- } \\
\text { occipital }\end{array}$} & $\begin{array}{l}\text { Changes from NS to } \\
S D-30 \text { minutes }\end{array}$ & $\begin{array}{l}r=0.210 \\
p=0.355\end{array}$ & $\begin{array}{l}r=0.264 \\
p=0.244\end{array}$ & $\begin{array}{l}r=0.189 \\
p=0.418\end{array}$ \\
\hline & $\begin{array}{l}\text { Changes with ToT - } \\
\text { NS }\end{array}$ & $\begin{array}{l}r=0.262 \\
p=0.213\end{array}$ & $\begin{array}{l}r=0.297 \\
p=0.156\end{array}$ & $\begin{array}{l}r=0.043 \\
p=0.840\end{array}$ \\
\hline & Changes with ToT -SD & $\begin{array}{l}r=0.144 \\
p=0.527\end{array}$ & $\begin{array}{l}r=0.270 \\
p=0.232\end{array}$ & $\begin{array}{l}r=-0.114 \\
p=0.626\end{array}$ \\
\hline
\end{tabular}

Changes from the normal sleep condition to the sleep deprivation condition in means SDLP and means EEG (averages obtained on the $30 \mathrm{~min}$ ) were obtained. We also quantified correlation between SDLP and EEG power spectra as changes over time after normal sleep (S1 versus S6) and after sleep deprivation (S1 versus S3). All these results were made including all participants for each EEG band (theta, alpha, beta) and for each cluster of electrodes (frontal, central, parieto-occipital) were made using the Spearman coefficient correlation. The $r$ value represents the coefficient of correlation between SDLP and the background EEG parameter considered. $p<0.05$ was considered as significant. ToT, Time-on-Task; NS, Normal sleep condition; SD, Sleep deprivation condition. 


\section{Discussion}

The main objective of the present study was to assess whether sleepiness exacerbates ToT effects both on EEG and driving performance in real driving conditions. We also wanted to know which EEG fluctuations are detectable after sleep deprivation during on-the-road driving.

The driving performance of completers, as measured by SDLP, was impaired by sleep deprivation and by ToT effects. The latter were more pronounced after sleep deprivation. Significant increases in alpha and theta power spectra were found after sleep deprivation compared to the normal sleep condition, indicated increased sleepiness and lowered arousal after sleep deprivation. The alpha and theta power spectra also varied significantly with ToT due to an increase in power over the first 40 minutes, followed by a decrease in power during the last 20 minutes of the driving test. The beta power increased with ToT, and this effect seemed most pronounced in the sleep deprivation condition although only a trend for Session by ToT interaction was found.

Comparisons of SDLP between completers and non-completers during the first part of the driving test showed that non-completers tended to drive worse than completers, irrespective of sleep condition. Effects of sleep deprivation and ToT on SDLP and EEG measures did not differ significantly between groups. Only, beta power varied differently with ToT between groups in the Frontal cluster while no significant variation with ToT was found in the Central and Parieto-Occipital clusters of electrodes. No relationship was found between EEG and driving performance. These findings are discussed in details below.

\subsection{Interaction effects between sleep deprivation and ToT on driving performance and EEG}

The driving performance varied significantly with ToT mainly in the sleep deprivation condition as shown by SDLP results. After normal sleep, SDLP was relatively constant over time, whereas it increased with ToT after sleep deprivation. At the start of the driving test (in S1) the impairing effects of sleep deprivation were minimal and increased until the midway turning point (in S2 and S3). Immediately following the turning point, the impairing effects of sleep 
deprivation were temporarily reduced (in S4), but reappeared (in S5) and increased even further in the last part of the driving test (in S6). We may suppose that the high demanding experimental condition (i.e sleep deprivation) led to fluctuations in abilities to maintain trajectory. Similar to the driving performance the EEG results revealed increased theta and alpha power spectra in all clusters in both conditions. The increased theta and alpha power spectra may reflect greater fatigue and sleepiness due to ToT. Increments in alpha and theta power were most pronounced during sleep deprivation. The finding that sleep deprivation exacerbated fatigue and sleepiness due to ToT effects is consistent with earlier laboratory works $(59,62,270)$ and support epidemiological studies that reported increased crash risk following extended driving after sleep deprivation (26-28). We also found an increased beta power spectrum in the Central cluster with ToT that may be linked to increased effort to maintain performance. This result is in accordance with previous findings after sleep deprivation (134).

\subsection{Comparison between completers and non-completers}

The additional analysis that investigated a Group effect led to a better understanding of performance decrement and EEG variations during driving after sleep deprivation. Concerning the driving performance, our results revealed that the SDLP tend to be higher for non-completers compared to completers in both sleep conditions. Although speculative (because we only reported a trend for the group by ToT interaction), the means in Figure 2 seem to support the idea that driving performance over time was worse in non-completers compared to completers. This result may explain why they had to stop driving in the sleep deprivation condition; non-completers participants were not able to keep their driving trajectories anymore.

Our results did not reveal any significant groups difference for alpha and theta, suggesting that non-completers were not sleepier than completers. However, the means in Figure 2 suggest that the alpha power spectrum was higher for non-completers than for completers in the sleep deprivation condition. The failure to obtain significant statistical results may be due to strong inter-individual difference within groups. Nonetheless, the decision to stop driving either by the 
participant himself or by the driver instructor for non-completers in the present study does not seem linked to higher sleepiness and fatigue state.

Both behavioral and EEG results contrast with those reported in the study of Akerstedt and colleagues (224) who investigated development of sleepiness indicators during an on-the-road driving task both during night- and daytime that could lead to the drive being prematurely terminated. In this study, the driving performance did not differ between the 8 participants who prematurely terminated the driving task and those who completed the task $(n=10)$. However, results revealed that participants who prematurely terminated the driving task showed significant higher levels of sleep intrusions (i.e KDS) on the EEG/EOG and that these sleepiness indicators were more pronounced during the night drive than during the day drive (224). The discrepancy between results from our study and those from the study of Akerstedt and colleagues may be explained by major differences in the design of both experiments. Firstly, in the study of Akerstedt and colleagues, the age range was larger and participants were older in mean (30$60 ; 46.6 \pm 8.8$ years old) than in the present study (23-45 years old; $26.9 \pm 3.4$ years old). It has been shown that younger participants are more likely to have driving incidents (52) and had higher SDLPs scores (271) than older (i.e experienced) drivers. Thus, the mean age of participants included in the present study could have lead to greater variation of the lateral position which may have contributes to detect driving performance difference between completers and non-completers. Secondly, the study of Akerstedt and colleagues was conducted in sleepier conditions than the present study (i.e during the afternoon and during the second half of the night). In addition, participants drove around one hour and a half while participants in the present study drove one hour. Altogether, the experimental conditions of the study of Akerstedt and colleagues could have exacerbated the sleepiness and fatigue status of participants. As a result, this might have led to higher levels of EEG sleep intrusions than in the present study that probably helped to highlight differences between completers and non-completers. Results of the present study also suggested occurrence of compensatory mechanisms due to effort in completers leading to less amounts of sleepiness indicators. Indeed, we found that, in the Frontal cluster, the beta power did not vary with ToT for non-completers while it was increasing with ToT for completers. 
Because it has been shown that elevated beta power at frontal sites reflects topdown low arousal compensation (272), this result could reflect differences in compensatory mechanisms in both groups due to effort $(115,133)$. Noncompleters may not have been able to invest more effort to finish the task as reflected by the lack of increased beta power with ToT in this group of participants.

\subsection{Sleep deprivation effects}

Our results showed that real driving performance, as measured by SDLP, is deteriorated after one night of total sleep deprivation. This result is in line with previous studies that reported driving performance decrement after sleep deprivation/restriction both in simulator $(52,54,63,273,274)$ and on-the-road driving settings $(51,64)$.

Regarding the EEG results, the present study showed that one night of sleep deprivation lead to increased alpha and theta power in all brain areas investigated (i.e. frontal, central and parieto-occipital). This is the first time that continuous EEG is recorded during a real driving task the morning after a night of total sleep deprivation. Previous studies have assessed EEG during real driving in the middle of the night, $(65,224,225)$ or during simulated driving $(52,63,115,121,225,228,249,275)$. Results from these studies suggest that an increased alpha and theta power is linked to an increased level of sleepiness $(52,63,65,121,225,249,275)$ and more fatigue $(110,115,228)$ as demonstrated by correlations with subjective measures of sleepiness. Laboratory investigations have shown that alpha is sensitive to attentional demands $(120,130,260)$. The theta band is linked to occurrence of a sleepiness state $(30,136,258,259)$. Our results may then reflect an increased state of sleepiness and more attentional demand after sleep deprivation than in the normal sleep condition. Contrary to our expectation, we did not find any significant effect of sleep deprivation on the beta frequency band compared to the normal sleep condition. 


\subsection{Time on Task effects}

The ToT effects on driving performance were few in the normal sleep condition, as SDLP was almost constant over time. This result differs from investigations using driving simulators, who revealed deterioration of performance with ToT even in well-rested conditions $(63,250)$. However, our results are consistent with those of two previous on-the-road driving studies. In the study of Sandberg and colleagues (65), no ToT effect on SDLP has been found considering both daytime and nighttime driving. In the study of Perrier and colleagues (In revision, Biol. Psychol.), only small variations of SDLP have been found over time. Altogether, our results and those of previous on-the-road driving investigations suggest that ToT effects are different within real driving setting compared with a simulator setting. Similar to the driving performance, the alpha power spectrum was almost constant over time in the normal sleep condition. Both results (i.e driving and alpha power) can be interpreted together. As the alpha power spectrum is known to reflect attentional processing $(118,120,123,124,133,262,276)$ and as both the alpha power spectrum and the driving performance stay almost at a constant level with ToT in the normal sleep condition, we may suppose that no additional demand was needed because the driving performance was not deteriorated by ToT and participants were able to maintain their trajectories.

In contrast, our results showed that the theta power increased even in the normal sleep condition until S4 compared to both the start and the end of the driving task. The increased theta power is in line with previous ToT effects reported during sustained attention (111) and simulated driving tasks $(63,115)$. However, after S4, the theta power spectrum was decreasing. As, the theta power is known to reflect sleepiness and fatigue states $(30,110,136,228,258,259)$; our results suggest that fatigue and sleepiness do not vary in one direction across the on-the-road driving task after a normal night of sleep. The latter differ from previous driving simulator results that showed a gradual increase of theta with ToT $(63,115)$. 


\subsection{Lack of correlation between SDLP and EEG measures}

Although we mentioned possible linkage between driving performance and fluctuations in background EEG fluctuations, we can not be sure that there is a direct link between both as we did not find any significant correlation between SDLP and background EEG. This lack of correlation is not in line with our expectation. Although some previous driving studies found a link between both parameters $(222,265)$, several other studies did not $(232-235)$. The individual variations in EEG parameters may be too large to find potential correlation with behavioral parameter (i.e SDLP). In addition, as fluctuations over time varied with the parameter considered (i.e EEG and SDLP), it could have been even more difficult to find significant correlation between both parameters across ToT.

\subsection{Limitations and future research}

One of the limitations of our study is the age range of participants that were included. Indeed, it has been shown that younger participants are more sensitive to the cumulative effects of sleep deprivation and ToT under simulated driving condition (52). Considering this previous finding, we can suppose that the same study conducted in older drivers could lead to less behavioral performance decrement with ToT after sleep deprivation. Lower markers of sleepiness and fatigue (i.e theta and alpha) and increased marker of compensation can also be expected. However, to our knowledge, these results about the cumulative effects of ToT and sleep deprivation have not been replicated in conditions of on-the-road driving.

Previous driving and EEG investigations that aimed to assess drivers fatigue and sleepiness demonstrated that the theta and alpha EEG bands $(226,236,277)$ were reliable measures that could be used as sleepiness indicators in on-board countermeasure devices. However, in the current study, our results showed that only the theta power spectrum increased with ToT in both conditions. In contrast, the alpha power spectrum and the SDLP were relatively constant over time after normal sleep. The theta power spectrum seems thus the most reliable measure to assess drivers fatigue status even in well-rested conditions. This information is important as few studies evaluated EEG fluctuations over time following sleep deprivation using on-the-road driving and then indicated the 
detectable and reliable measures of drivers fatigue and sleepiness. Future investigations that aimed to develop drowsy or fatigue detection systems adapted to the real highway driving conditions should take this information into account.

Our study revealed for the first time in on-the-road driving condition that sleep deprivation exacerbate driving impairment as reflected by SDLP as well as fatigue and sleepiness (i.e theta and alpha power spectra) as a function of ToT effects. These effects were particularly true for the theta power spectrum that should be taken into account for the development of future status drivers system. Our results also suggested that participants who completed both driving sessions (i.e completers) put more effort across the task compared to those who prematurely terminated the drives (i.e non-completers), as reflected by their increased beta power spectrum in the Frontal cluster compared to non-completers. 



\section{Chapter 6 \\ Specific electroencephalographic sleep pattern in the prefrontal cortex in primary insomnia: a spectral analysis}

Published as:

Perrier, J., Clochon, P., Bertran, F., Couque, C., Bulla, J., Denise, P., \& Bocca, M.-L. (2015). Specific EEG Sleep Pattern in the Prefrontal Cortex in Primary Insomnia. PLoS ONE, 1O(1), e0116864. 


\begin{abstract}
The aim of the current investigation was to assess the specific prefrontal activity compared to that in the other main cortical areas in primary insomnia patients and in good sleepers. Fourteen primary insomnia patients and eleven good sleepers were included in the analysis. Participants completed one night of polysomnography in the sleep lab. Power spectra were calculated during NREM (Non-Rapid Eye Movement) and REM (Rapid Eye Movement) sleep at prefrontal, occipital, temporal, and central electrode positions. During NREM sleep, the power spectra did not differ between groups in the prefrontal cortex; while primary insomnia patients exhibited a higher beta power spectrum and a lower delta power spectrum compared to good sleepers in other areas. During REM sleep, the beta1 power spectrum was lower in the prefrontal cortex in primary insomnia patients compared to good sleepers; while no significant difference between groups was obtained for the other areas. The present study shows a specific prefrontal sleep pattern during the total sleep period. In addition, we suggest that primary insomnia patients displayed a dysfunction in the reactivation of the limbic system during REM sleep and we provide additional arguments in favor of a sleep-protection mechanism displayed in primary insomnia patients.
\end{abstract}




\section{Introduction}

Between 5 and $30 \%$ of the general population complains of insomnia, which makes this pathology one of the most prevalent sleep disorders $(6,7,170)$. It is a public health issue as the annual direct and indirect costs have been globally estimated at $\$ 1253$ in the United States (15) and at \$5010 per year in the province of Quebec, Canada (16). Among the different types of insomnia defined by the DSM-IV, primary insomnia (PI) is a complaint of non restorative and/or insufficient sleep with difficulty initiating and maintaining sleep, and early morning awakenings associated with daytime consequences, without comorbidity. Using this definition, PI affects 2-4\% of the general population (141).

Despite the socioeconomic burden and the daytime consequences of insomnia (16), this pathology and its sleep characteristics are poorly understood $(80,81)$. Polysomnography (PSG) investigations in PI have not consistently revealed modifications in sleep architecture. PSG derived sleep variables produced far fewer pronounced differences than expected in good sleepers when compared to the subjective estimates of sleep quality in patients (210). A more detailed approach such as the investigation of power spectra analysis (PSA) is considered more promising for better understanding the pathophysiology of PI (79). A general finding in PSA is increased beta activity during NREM in PI patients compared to good sleepers $(84-87,278)$, which reflects cortical hyperarousal in PI $(81,279)$. In contrast to NREM findings, REM investigations have led to inconsistent results as significant differences in the beta power spectrum between PI patients and good sleepers were found in two studies $(86,87)$, while no group differences were found in two others $(84,85)$.

Since previous studies of spectral analysis during sleep have not compared the cortical activity between various brain areas, no specific cortical activity during sleep in one area compared to the others has been studied. Thus, further PSA investigations will be useful to better understand the spectral modifications that could occur during sleep in specific cortical areas in PI as well as its physiopathology. Several neuropsychological studies have revealed that PI patients had impaired performance in tests involving the prefrontal cortex (68,280-282), and neuroimaging studies have shown brain function and 
morphology alterations in the prefrontal cortex $(72,77,88)$, respectively. As the prefrontal cortex has a major role in mediating sleep physiology, e.g. deactivation during the transition between waking state and NREM, increasing this deactivation with the deepening of NREM sleep and reactivation during sleep $(90,283,284)$, we can suppose that alterations or modifications in the prefrontal cortex could be present during the sleep period in PI patients. The objective of the current investigation was thus to assess the sleep EEG power spectra in PI patients and in good sleepers in four main cortical areas (temporal, central, occipital, and prefrontal) and to compare the EEG power spectrum in other areas (occipital, central, and parietal) to those in the prefrontal area.

\section{Methods}

\subsection{Participants}

The data presented in this paper are part of a study whose results concerning behavioral (driving performance) data were recently published (144). The polysomnographies were recorded the night before the driving evaluation for all participants. Twenty-one PI patients and sixteen good sleepers were included. Sleep architecture was analyzed for 17 PI patients and 11 good sleepers, but after inspection for spectral analysis, due to strict artifact rejection (e.g. eye movements), not all recordings could be analyzed. Consequently, both sleep architecture and spectral analysis are presented here for the same participants remaining, i.e. 14 PI patients ( 7 men and 8 women; mean age $=47 \pm 17$; age range $=24-74$ years) and 10 good sleepers ( 4 men and 7 women; mean age $=46$ \pm 15 ; age range $=23-64$ years $)$.

After a telephone interview, the participants from both groups had a medical interview by a sleep clinician to ascertain: i) sleeping difficulties and diagnosis of DSM-IV insomnia for the insomnia patient group; ii) good physical condition; the absence of sleep, alertnes; neurological, cardiovascular, respiratory, hepatic, renal, or metabolic disorders; presence of poor hygiene or habitual abnormal sleep patterns (e.g. night or shift work) for the good sleeper group. 
The participants included in the insomnia group had to meet the following inclusion criteria according DSM-IV primary insomnia criteria: (a) presence of a subjective complaint of insomnia defined as difficulty initiating (sleep onset latency (SOL), $>30 \mathrm{~min}$ ) and/or maintaining (time awake after sleep onset $>30$ $\mathrm{min})$ sleep; (b) early awakening $(<6.5 \mathrm{~h}$ of sleep or waking up earlier than the desired wake up time); (a) and (b) had to occur at least three nights per week; (c) insomnia duration of at least 6 months; (d) insomnia or its perceived consequences causing marked distress or significant impairment of occupational or social functioning (problem of concentration); and (e) presence of a subjective complaint of at least one negative daytime consequence attributed to insomnia (fatigue, mood disturbances).

The exclusion criteria were: (a) significant current medical or neurological disorder that could compromise sleep; (b) major psychopathology that could induce insomnia; (c) psychotropic or other medications consumption known to alter or induce sleep; (d) poor hygiene or habitual abnormal sleep patterns (e.g. night or shift work); and (e) another sleep disorders (assessed by polysomnography) such as sleep apnea (apnea-hypopnea index $>10$ ), or periodic limb movements during sleep (myoclonic index with arousal $>10$ ). All insomnia and good sleepers participants were excluded if they: i) had a current or past dependence on alcohol, opiates, benzodiazepines, or any illicit drugs; ii) smoked more than five cigarettes per day; iii) drank more than 28 units of alcohol per week or iv) consumed more than $150 \mathrm{mg}$ of caffeine per day. All participants had normal or corrected to normal vision (visual acuity greater than or equal to $7 / 10$ ).

The study was granted ethical approval by the Caen Northwest III ethics committee and by the Health Ministry (number DGS 2005/0388). Each participant gave written informed consent in accordance with the requirements of the committee.

\subsection{Polysomnography}

A standard laboratory procedure was conducted for all participants for one experimental night.

Sleep was recorded using a polysomnography ambulatory monitoring machine (Medatec Dream). A standard montage of polysomnography was used 
including 8 EEG channels (FP1, FP2, C3, C4, O1, O2, T3, T4 referenced on linked mastoid A1 and A2), 2 electrooculogram (EOG) channels, and one submental electromyogram channel. This montage was complemented by recordings from the left and right anterior tibialis muscle, recordings of nasal/oral airflow, thoracic and abdominal effort, body position) and oximetry. All PSG were scored according to the standard criteria (196) by experienced sleep specialists $(\mathrm{CC}, \mathrm{FB})$.

The objective measures of sleep included sleep onset latency (SOL, min), waking after sleep onset (WASO, min), total sleep time (TST, min), sleep efficiency (SE, \%), total time (min), latencies ( $\mathrm{min})$, and percentage (\%) of stages 1,2 , and $3+4$, and rapid eye movement (REM).

\subsection{Spectral analysis}

The EEG data were filtered with a band pass filter of $0.16-70 \mathrm{~Hz}$ and sampled at $200 \mathrm{~Hz}$. Computerized spectral analysis was performed with fast Fourier transformation (FFT) on the all-night filtered EEG after elimination of epochs with artifacts (eye movement, electrocardiogram, electromyogram or movementrelated artifacts). Spectral analysis was performed on 5.12-s epochs and on the 8 channels recorded (FP1, FP2, C3, C4, O1, O2, T3, T4, referenced on linked mastoid A1 and A2) to investigate the difference in EEG power spectra in different areas. Before computing the FFT, the data were tapered with the Hamming window. The FFT was computed on artifact-free epochs. The FFT was carried out on each sleep stage on the total number of epochs corresponding to the maximal number of artifact-free epochs observed in all subjects. The relative power spectrum was obtained in frequency bands as follows: delta $(1.5-4 \mathrm{~Hz})$; theta $(4-7.5 \mathrm{~Hz})$; alpha (separated in alpha1, alpha2, and alpha 3 and aggregated) (7.5-12.5 Hz); sigma (12.5-14 Hz); beta (separated in beta1, beta2, and beta 3 and aggregated) (14-30 Hz). All night power averages were obtained separately for stage 2 , stage $3+4$, and REM sleep.

\subsection{Statistical analysis}

Statistical analyses of EEG data were performed with SAS (SAS Institute Inc., 9.3) and R (3.0.1) software (285). Polysomnography variables were compared using a variance analysis with the GLM procedure with comparison between groups (PI 
patients vs good sleepers). For spectral analysis, we used an analysis of variance (ANOVA) with two factors, including group and areas (prefrontal vs other areas which correspond to the mean of the three other areas). Post hoc comparisons between groups and areas for significant interactions were then conducted with Tuckey $t$-tests to control for multiple comparisons. For the spectral analysis, only results of the group effect and the group by area interactions are described in the results section. The significance level was set at $p<0.05$.

Moreover, we calculated effect sizes using partial omega squared (286). These effect sizes were reported for group comparison for the sleep architecture results and for group comparisons and group by area interactions for the spectral analysis results.

\section{Results}

Participant characteristics are given in Table 1 for each group.

Table 1. Baseline characteristics of participants

\begin{tabular}{cccc} 
Questionnaires & $\begin{array}{c}\text { Insomnia patients }(\mathbf{n}=17) \\
\text { Mean } \\
\text { (SD) }\end{array}$ & $\begin{array}{c}\text { Good sleepers }(\mathbf{n}=11) \\
\text { Mean } \\
\text { (SD) }\end{array}$ & p value \\
\hline ISI & $18.5(4.80)$ & $3.29(1.38)$ & $0.006^{* *}$ \\
PSQI & $10.86(2.67)$ & $3.90(2.38)$ & $<0.001^{\text {*** }}$ \\
$\begin{array}{c}\text { Horne and } \\
\text { Ostberg }\end{array}$ & $56.92(10.24)$ & $58.80(9.80)$ & 0.66 \\
Age & $47(16.82)$ & $44.2(14.96)$ & 0.68 \\
Sex & $5 \mathrm{M} / 9 \mathrm{~F}$ & $4 \mathrm{M} / 6 \mathrm{~F}$ & $/$
\end{tabular}

Between-group analyses (unpaired $t$ tests). Equality of variance correction was applied. SD: Standard Deviation, ISI: Insomnia Severity Index, PSQI: Pittsburg Sleep Quality Index. ${ }^{* *} p<0.05 ;{ }^{* *} p<0.001$. 


\subsection{Polysomnography}

Polysomnography results are presented in Table 2. Statistical results revealed that the sleep efficiency was lower and that the number of WASO was higher in PI patients compared to good sleepers.

Table 2. Sleep characteristics of participants

\begin{tabular}{|c|c|c|c|c|}
\hline $\begin{array}{c}\text { Sleep } \\
\text { parameters }\end{array}$ & $\begin{array}{l}\text { Insomnia patients ( } \\
\quad=14 \text { ) Mean (SD) }\end{array}$ & $\begin{array}{l}\text { Good sleepers ( } n \\
=10 \text { ) Mean (SD) }\end{array}$ & $\begin{array}{c}p \\
\text { value }\end{array}$ & $\begin{array}{c}\omega_{\mathrm{p}}^{2}[\mathrm{Cl} \\
90 \%]\end{array}$ \\
\hline TSP (min) & 457.26 (23.03) & $4451.68(48.50)$ & 0.71 & $\begin{array}{c}-0.037 \\
{[0.00 ;} \\
0.13]\end{array}$ \\
\hline TST (min) & $382.39(544.71)$ & 408.42 (47.97) & 0.19 & $\begin{array}{c}0.034 \\
{[0.00} \\
0.28]\end{array}$ \\
\hline SE (\%) & $83.73(9.45)$ & 90.05 (7.07) & $0.071^{*}$ & $\begin{array}{c}0.098 \\
{[0.00} \\
0.35]\end{array}$ \\
\hline $\begin{array}{l}\text { REM Latency } \\
\text { (min) }\end{array}$ & $106.20(38.85)$ & $101.29(40.50)$ & 0.78 & $\begin{array}{c}-0.040 \\
{[0.00 ; 0.11]}\end{array}$ \\
\hline $\begin{array}{c}\text { Number of } \\
\text { WASO (>1min) }\end{array}$ & $09.36(4.83)$ & $5.89(2.56)$ & $0.051^{*}$ & $\begin{array}{c}0.12[0.00 \\
0.37]\end{array}$ \\
\hline Stage 1 ( $\%$ TST) & $10.46(4.90)$ & $12.062(3.35)$ & 0.24 & $\begin{array}{c}0.018 \\
{[0.00 ; 0.25]}\end{array}$ \\
\hline Stage 2 (\% TST) & $43.44(10.44)$ & $43.89(7.93)$ & 0.91 & $\begin{array}{c}-0.042 \\
{[0.00 ; 0.05]}\end{array}$ \\
\hline SWS (\% TST) & 29.01(10.55) & $26.00(6.00)$ & 0.42 & $\begin{array}{c}-0.014 \\
{[0.00 ; 0.20]}\end{array}$ \\
\hline REM (\% TST) & $17.09(5.94)$ & $17.15(4.16)$ & 0.85 & $\begin{array}{c}-0.042 \\
{[0.00} \\
0.086]\end{array}$ \\
\hline
\end{tabular}

${ }^{*} 0.1>p>0.05$; Between groups analysis using two-way ANOVA (GLM procedure), insomnia patients vs good sleepers. TSP: Total Sleep Period, TST: Total Sleep Time, SE: Sleep efficiency, REM: RapidEye Movement, WASO: Waking After Sleep Onset, SWS: Slow Wave Sleep, SD:Standard Deviation, $\omega^{2}{ }_{p}$ : partial omega squared. 


\subsection{Spectral analysis}

Significant results of the power spectral analysis for the group effects and the group by area interaction for both NREM sleep and REM sleep are summarized in Table 3. Statistical results for the post hoc comparisons are given in the text. To focus the statistical description on the spectral changes in PI compared to the good sleepers related to brain areas, only the significant group effects and the post hoc corresponding to the significant group by area interactions are detailed for each sleep stage and each frequency band in the following section.

\section{Stage 2}

For both the delta and the beta bands, statistical analysis revealed a group by area interaction but no significant difference between groups. For the delta band, post hoc comparisons revealed no significant difference either between groups or between areas (See Figure 1). For the beta band, post hoc comparisons revealed that good sleepers displayed a significantly lower power spectrum in the other areas than in the frontal area $(p=0.030)$ (See Figure 2).

\section{$\underline{\text { Stage } 3+4}$}

Statistical results for the group effect revealed that PI patients displayed significantly higher alpha and sigma power spectra than good sleepers and a lower delta with a trend. For the delta band, post hoc comparisons of the significant group by area interaction revealed that PI patients displayed a significantly lower power spectrum than good sleepers in the "other areas" $(p=0.039)$ whereas no significant difference between groups was found for the prefrontal area $(p=1.0)$ (See Figure 3). For the sigma band, post hoc comparisons of the significant group by area interaction revealed that PI patients displayed significantly higher power spectrum in the "other areas" $(p=0.056)$, whereas no significant difference between groups was found for the prefrontal area $(p=1.0)$ (See Figure 4). For the beta band, post hoc comparisons of the significant group by area interaction revealed that PI patients displayed a significantly higher power spectrum in the "other areas" $(p=0.013)$, whereas no significant difference between groups was found for the prefrontal area $(p=0.38)$ (See Figure 5).

\section{$\underline{\text { REM stage }}$}


For the beta1 band, post hoc comparisons revealed that the lower power spectrum of the PI patients in the prefrontal area was near significant level $(p=0.060)$ (See Figure 6).

Table 3. Significant results of the power spectral analysis

\begin{tabular}{|c|c|c|c|c|c|c|c|}
\hline \multirow[t]{2}{*}{ Stages } & \multirow[t]{2}{*}{ Bands } & \multicolumn{2}{|c|}{$\begin{array}{l}\text { Group effect } \\
\text { DF=3 }\end{array}$} & \multicolumn{2}{|c|}{$\begin{array}{l}\text { Group*Areas } \\
\text { Effect DF=1 }\end{array}$} & \multicolumn{2}{|l|}{$\begin{array}{l}\omega_{\mathrm{p}}^{2} \\
{[\mathrm{Cl} 90 \%]}\end{array}$} \\
\hline & & & $p$ & $F$ & $p$ & Group effect & $\begin{array}{l}\text { Groups }{ }^{*} \text { Areas } \\
\text { effect }\end{array}$ \\
\hline Stage & Delta & 0.02 & 0.895 & 4.55 & $0.039^{* *}$ & $-0.021[0.00 ;$ & $0.069[0.003$ \\
\hline \multirow[t]{3}{*}{2} & & & & & & $0.03]$ & $0.23]$ \\
\hline & Beta & 0.02 & 0.90 & 8.60 & $0.005^{* *}$ & $-0.021[0.00$ & $0.14[0.29$ \\
\hline & & & & & & $0.028]$ & $0.31]$ \\
\hline
\end{tabular}

Stage Delta $3.56 \quad 0.066^{*} \quad 4.16 \quad 0.048^{* *} \quad 0.051[0.00 ; \quad 0.062[0.00 ;$

$3+4$

$\begin{array}{llllllll} & & & & & 0.21] & 0.22] \\ \text { Alpha } & 6.48 & 0.015^{* *} & 0.58 & 0.45 & 0.10[0.014 ; & -0.009[0.00 \\ & & & & & 0.27] & 0.11] \\ & & & & & & \\ \text { Sigma } & 5.95 & 0.019^{* *} & 6.37 & 0.015^{* *} & 0.093[0.011] & 0.10[0.013 ; \\ & & & & & & 0.27] \\ \text { Beta } & 1.29 & 0.26 & 11.65 & 0.0014^{* *} & 0.0059[0.00 ; & 0.18[0.053 ; \\ & & & & & 0.14] & 0.36] \\ \text { Beta1 } & 3.06 & 0.087^{*} & 3.66 & 0.062^{*} & 0.041[0.00 ; & 0.05[0.00 ; \\ & & & & & 0.20] & 0.21]\end{array}$

${ }^{*} 0.1>p>0.05$; ${ }^{*} p<0.05$; Groups effect, insomnia patients versus good sleepers; Groups ${ }^{*}$ Areas effect, interaction between group effect and frontal versus others scalp areas effect; REM, Rapid Eye Movement; DF, Degree of Freedom, $\omega^{2}$ : partial omega squared. 


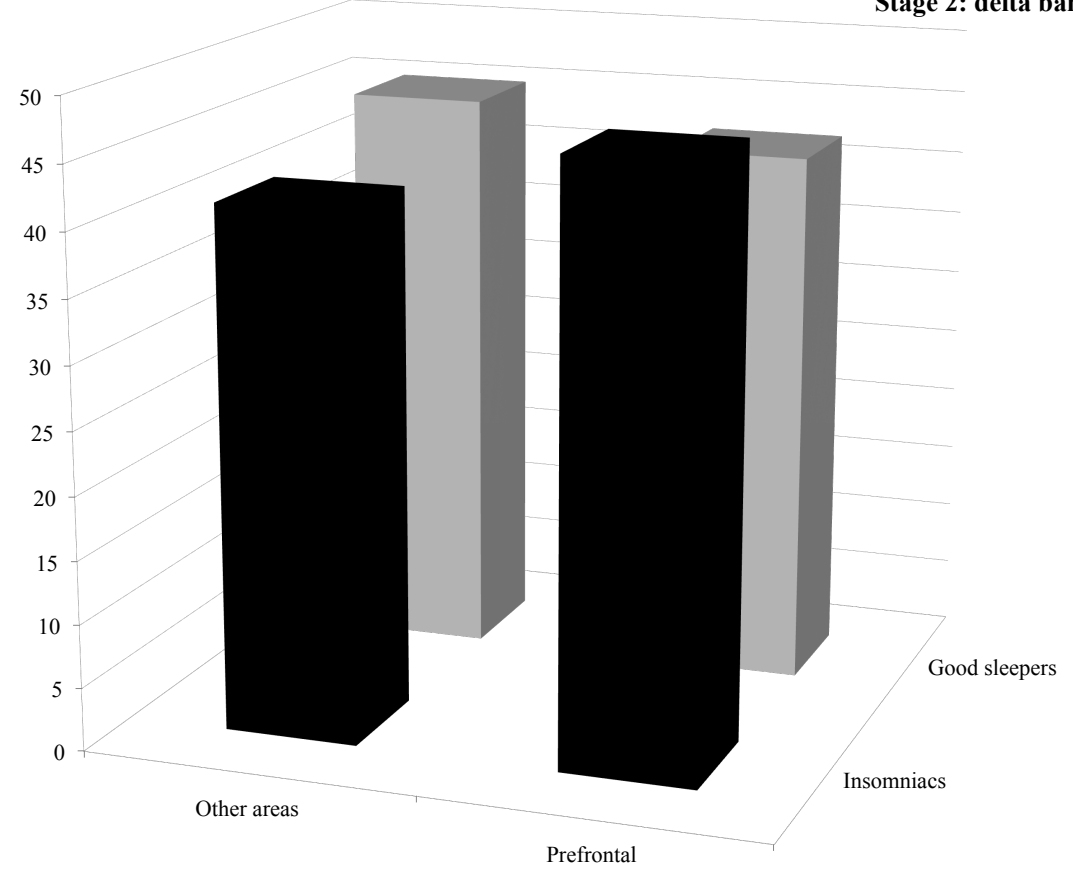

Figure 1. Relative power spectra for the delta band during stage 2 for both groups (insomnia patients and good sleepers) and each area (others vs prefrontal). ${ }^{* *} p<0.05$. 


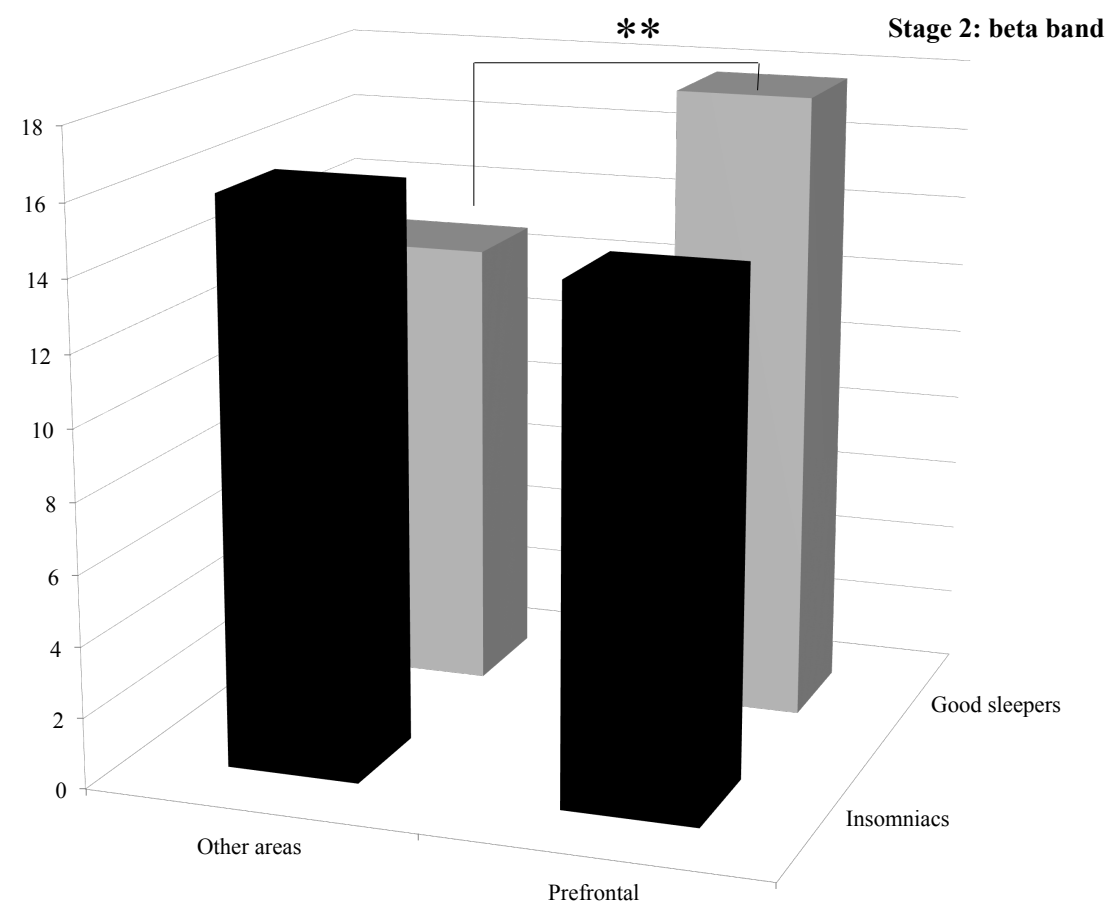

Figure 2: Relative power spectra for the beta band during stage 2 for both groups (insomnia patients and good sleepers) and each area (others vs prefrontal). ${ }^{* *} p<0.05$. 


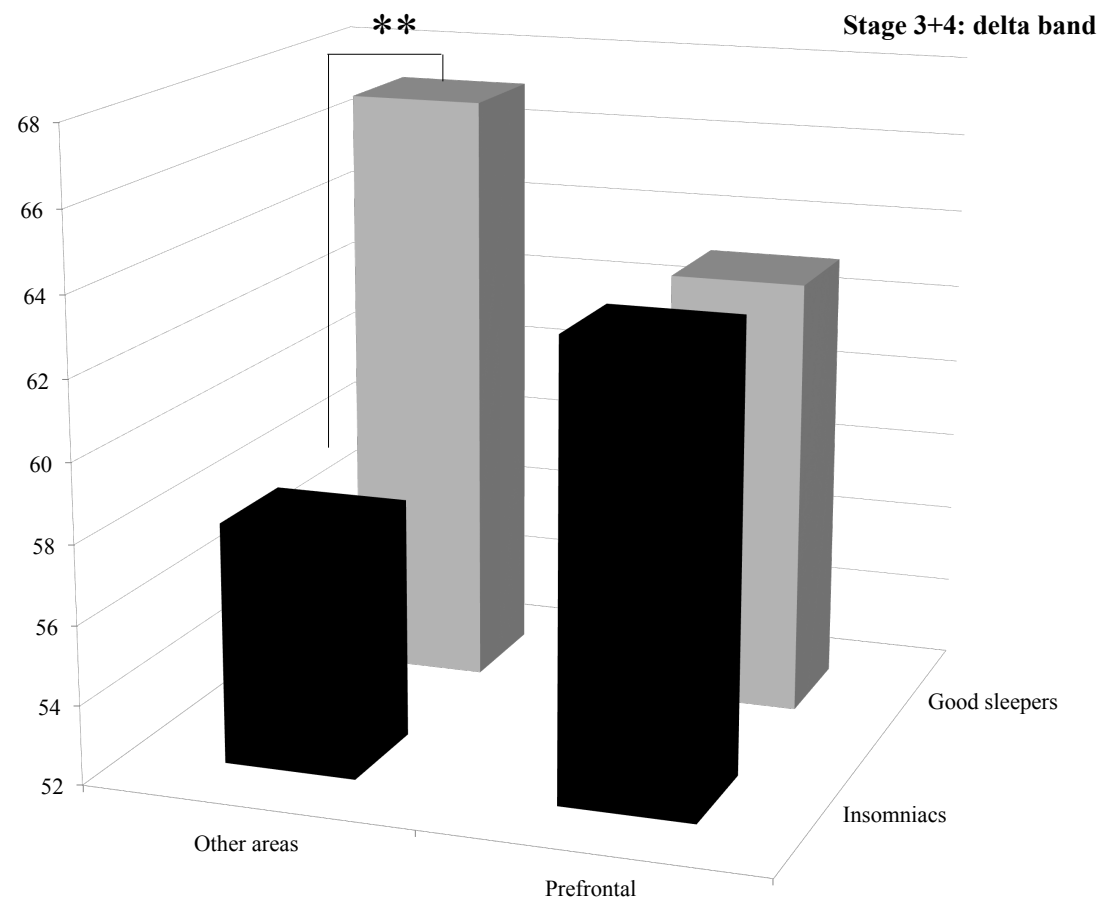

Figure 3: Relative power spectra for the delta band during stage $3+4$ for both groups (insomnia patients and good sleepers) and each area (others vs prefrontal). ${ }^{* *} p<0.05$. 


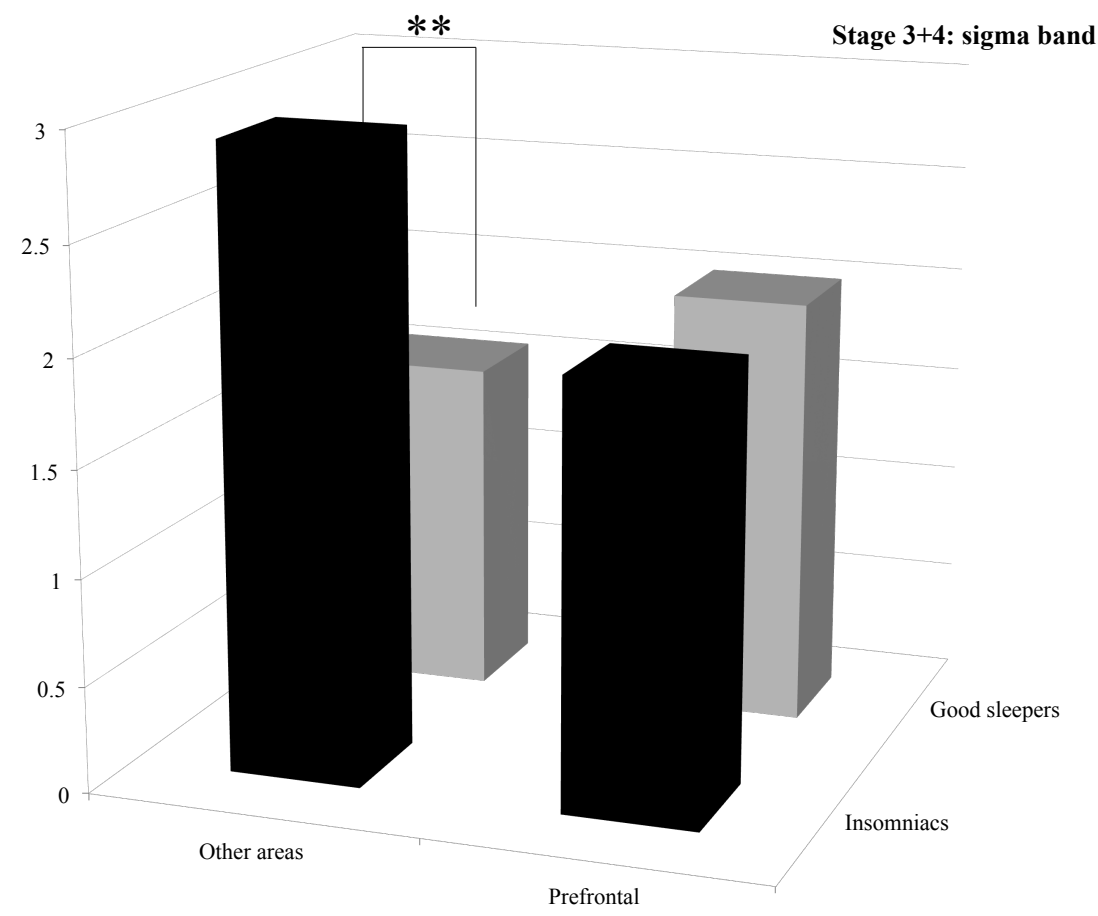

Figure 4: Relative power spectra for the sigma band during stage $3+4$ for both groups (insomnia patients and good sleepers) and each area (others vs prefrontal). ${ }^{* *} p<0.05$. 


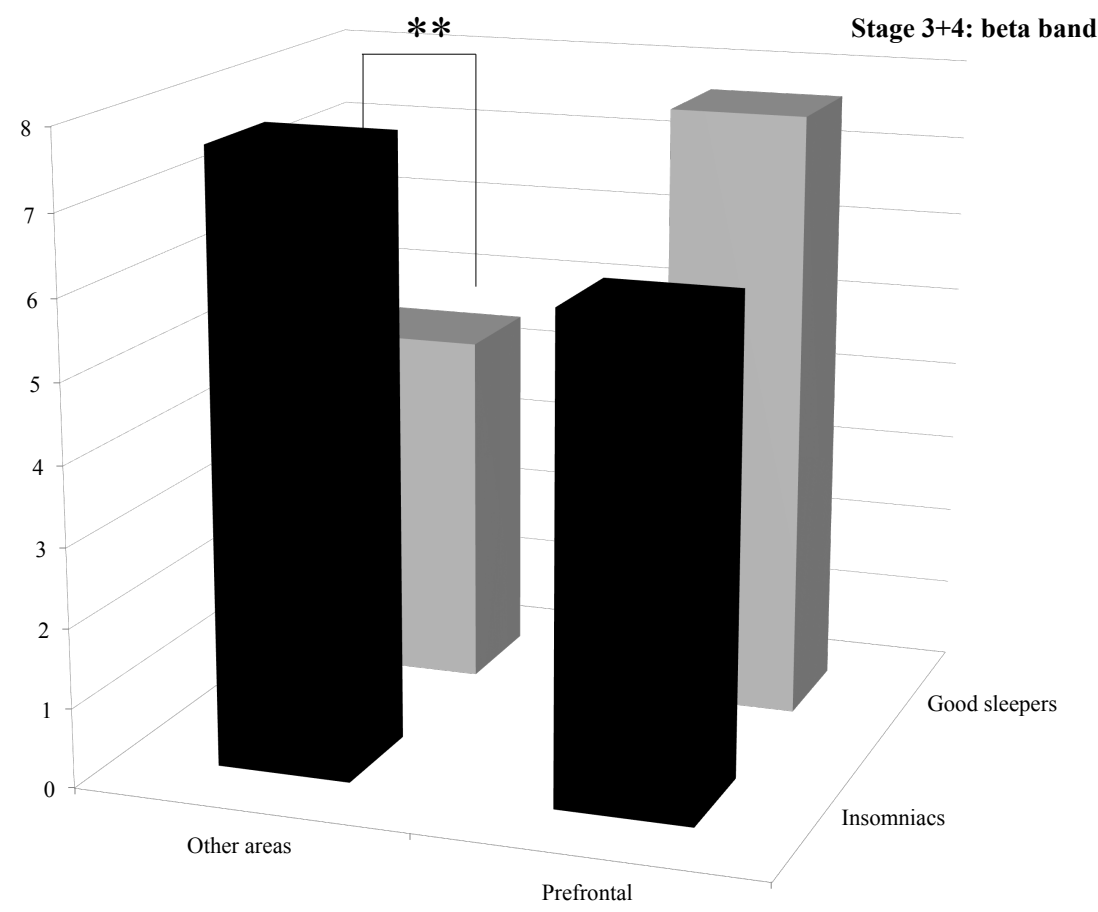

Figure 5: Relative power spectra for the beta band during stage $3+4$ for both groups (insomnia patients and good sleepers) and each area (others vs prefrontal). ${ }^{* *} p<0.05$. 


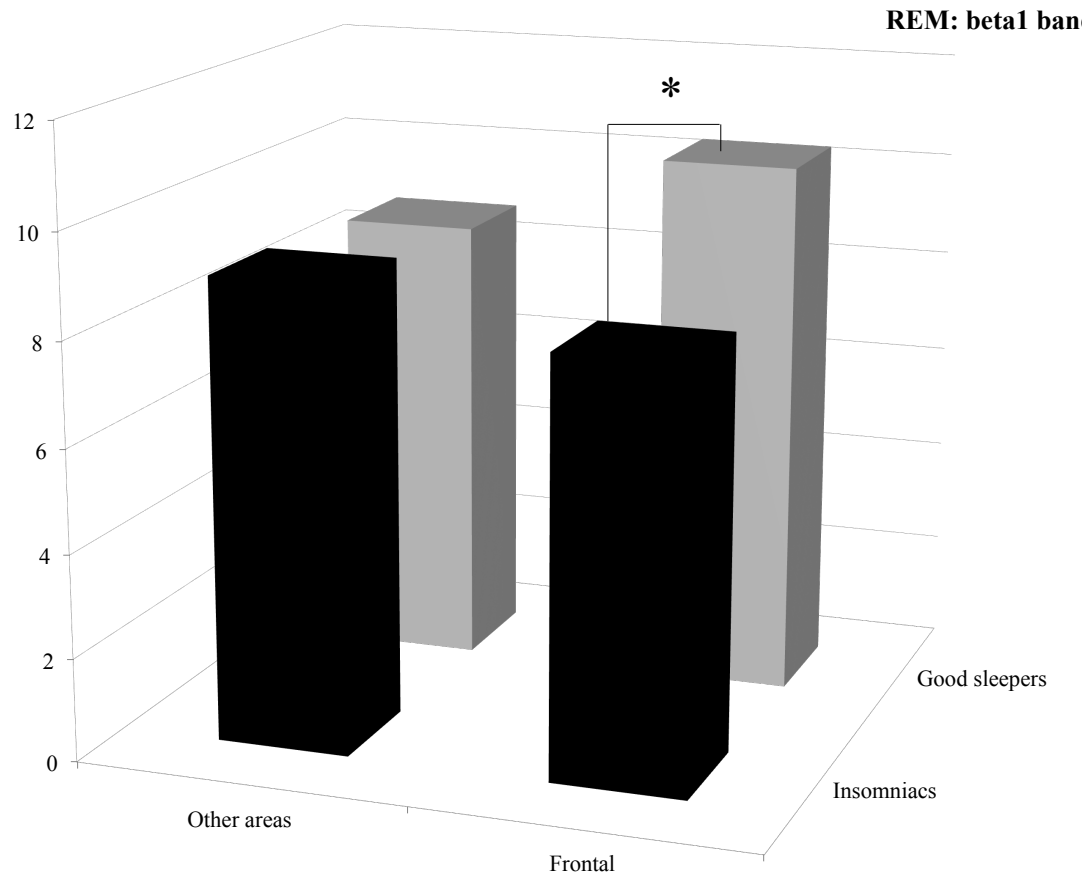

Figure 6: Relative power spectra for the beta1 band during stage REM for both groups (insomnia patients and good sleepers) and each area (others vs prefrontal). ${ }^{*} 0.1>p>0.05$. 


\section{Discussion}

The aim of the current investigation was to evaluate whether, in PI patients, the prefrontal cortex displayed a specific sleep pattern compared to good sleepers. The main results showed that: i) the prefrontal cortical activity in PI patients differs from that recorded in the other cortical areas; ii) this specific difference between cortical activity in areas in PI patients was observed during both NREM and REM sleep; iii) the relative beta power spectrum in PI patients was higher compared to good sleepers for the other cortical areas and this result remained constant throughout NREM sleep (stage 2 and stage $3+4$ ).

The specific prefrontal activity in PI patients was revealed during NREM sleep (stage 2 and $3+4$ ) by a concomitant lack of difference between groups for the delta and beta power spectra in the prefrontal area with the presence of cortical activity differences between groups in other areas. We also found cortical activity differences between groups for the sigma band during stage $3+4$ in the other areas, while no group difference was revealed for the prefrontal area. During REM sleep, the only difference between the two groups was found for the prefrontal electrodes, as we observed that PI patients displayed fewer beta1 power spectra than good sleepers in this cortical area. To our knowledge, this is the first report about a specific prefrontal sleep pattern compared to other cortical areas in PI patients.

Our NREM findings for the other areas (occipital, central, and temporal areas) revealed that PI patients displayed: i) higher beta and lower delta power spectra, and; ii) a higher sigma power spectrum compared to good sleepers. These results are in agreement with previous reports $(84-87,278)$. Nevertheless, the present study is the first to describe simultaneously a higher beta power spectrum throughout the entire NREM sleep (i.e stage 2 and stage $3+4$ ) in PI patients. It is important to note that our participants had been free of medications for at least two years. Therefore, our results showing an increased sigma power spectrum could not be linked to the what is called the "GABA-Benzodiazepine signature" which refers to suppression of frequencies below $10 \mathrm{~Hz}$ and an increase in sigma 
band power $(287,288)$. Our results can be interpreted as a hyperarousal state throughout NREM sleep in PI.

Our REM results, demonstrated a lower beta1 value in the prefrontal area in PI patients compared to good sleepers, but no difference between groups in the other cortical areas (central, temporal, occipital). The lack of difference in activity between groups in the central area in the current study is in line with the results of Spiegelhalder and colleagues (85). Our findings in the prefrontal area contrast with those of Merica and colleagues (86), who found increased beta and gamma band (i.e higher frequency band) activity in F4 in PI patients compared to good sleepers. However, the authors were not convinced by their REM results as they mentioned possible NREM sleep intrusions in REM sleep leading to unexpected results (increased in higher frequency bands) during this period.

The specific sleep pattern revealed in the prefrontal area compared to other cortical areas during NREM and REM stages in PI patients extends previous findings which revealed frontal modifications or alterations in PI during the waking state. Indeed, by using neuroimaging, previous studies showed that PI patients displayed: i) higher general brain metabolism during the waking state and sleep (89); ii) a reduced relative metabolism in the prefrontal cortex in comparison with other brain areas during the waking state (89); and iii) a specific hypoactivation of the prefrontal cortex during a letter fluency task in comparison to good sleepers (77).

Altogether, our NREM and REM results indicate that PI patients displayed a specific dysfunction in the reactivation of the limbic system during the REM period rather than a global prefrontal dysfunction during sleep. During REM sleep, in the prefrontal cortex, there is a reactivation (i.e an increase in the cortical activity) of what is called the "anterior paralimbic REM activation area" $(289,290)$, while other parts of the prefrontal cortex remain deactivated $(289,291)$. In contrast, during NREM sleep, the entire prefrontal cortex is deactivated (including the anterior paralimbic REM activation area). Our results may then be interpreted as follows: i) the hypoarousal, reflected by less beta1 activity during REM sleep in PI patients in prefrontal electrodes, may be linked to a problem in the reactivation of the anterior paralimbic area during REM sleep; ii) the lack of hyperarousal, reflected by no difference in beta activity between groups 
during NREM sleep in prefrontal electrodes, may be linked to the fact that the entire prefrontal cortex is deactivated during this sleep stage.

Our results showing a concomitant greater sigma and alpha power spectra during the NREM sleep in PI patients compared to good sleepers seem to support the idea that PI patients display more sleep-protective mechanisms than good sleepers. The sleep microstructure modifications linked to PI and their interpretations are complex, and varied results can be found in the literature (See (79) for a review). Among them, a consistent finding in PI patients is a higher cognitive brain state during sleep. However, it is not clear whether PI patients display more sleep-promoting mechanisms compared to good sleepers $(292,293)$. The higher sigma power spectrum during stage $3+4$ in insomnia patients compared to good sleepers found in the other areas can be viewed as an increase in sleep stability (284). In a recent review, authors underlined the role of the alpha rhythm in inhibition mechanisms in reflecting a "closed thalamic gate" (120). Consequently, our findings suggest that PI had more sleep-promoting mechanisms (reflected by greater sigma and alpha rhythms) than good sleepers. This can be linked to compensatory mechanisms against the hyperarousal state (reflected by an increase in beta rhythm).

This study has some limitations, in particular our small sample size. However, the patients included were carefully diagnosed for primary insomnia, were without comorbidity, and without concomitant drug intake. Complementary investigations should be performed to extend our findings. In addition, further studies are also needed in other subtypes of insomnia in order to assess whether the specific prefrontal activation pattern found here is also found in other subtypes. Such studies are also needed to better understand the pathophysiology of insomnia. Additionally, sleep recording was performed during a single night of polysomnography. It is well known that participants sleep architecture during the first night in a sleep laboratory environment differs from subsequent nights, which is called "the first night effect" (FNE). Of note, it has been shown that in good sleepers the TST, the time spent in REM sleep, and sleep efficiency are lower and the REM latency and the number of WASO are increased $(294,295)$ compared to subsequent nights. In the current investigation, we assumed that the relative high amount of stage 1 found in good sleepers is due to this FNE as it leads to more 
WASO. In the insomnia patient group, the high amount of SWS found can be explained by the reverse first night effect (RFNE), already described in these patients $(294,296)$. Furthermore, it has been shown that insomnia patients sleep better at the sleep laboratory than at home (296). Consequently, the SWS can be increased and thus here appeared unusually high for primary insomnia patients.

In conclusion, the present study provides evidence, for the first time, of a prefrontal sleep pattern that differs substantially from that in other cortical areas during both NREM and REM sleep. Altogether, our results suggest that PI patients display a specific dysfunction in the reactivation of the limbic system during the REM period. Our results also provide additional arguments in favor of a sleep-protection mechanism displayed by PI patients in addition to their classic cortical signature of hyperarousal. 
Chapter 7

General discussion 
In this dissertation, the five experimental chapters were designed to study daytime deficits as well as EEG fluctuations following sleep deprivation and PI. We described new findings on the daytime deficits of PI patients, particularly those of vigilance and conflict resolution. Our results showed a driving performance decrement in PI patients in the condition of simulated monotonous driving, which contrasts with the lack of impairment observed in an on-the-road driving study. In addition, results from the ANT revealed that compared to good sleepers, PI patients had a longer overall reaction time and a longer reaction time in the incongruent flankers condition compared to other flanker conditions. However, no performance impairment was found in the PVT in PI patients compared to good sleepers. We also investigated spectral EEG fluctuations during the waking state and during sleep in PI patients. The EEG fluctuations over time during daytime on-the-road driving were smaller in PI patients compared to those in good sleepers. The sleep spectral investigation in PI patients indicated a specific EEG sleep pattern in the prefrontal cortex compared to other cortical areas (i.e. parietal, occipital and central) during both NREM and REM sleep. Finally, we demonstrated that sleep deprivation deteriorated the on-the-road driving performance and exacerbated the ToT effects on both driving performance and EEG. In the present chapter, our major findings will be discussed.

The aims of the current dissertation were as follows and are discussed separately below:

- Evaluate daytime performance (as a function of Time on Task) in PI patients and in healthy volunteers after sleep deprivation,

- Investigate the pathophysiology of PI during both daytime and the sleep periods by using EEG to determine brain activity modifications.

\section{Attention and vigilance daytime deficits in primary insomnia}

Attention and vigilance performances in PI are described in Chapters 2, 3 and 4. The driving performances of PI patients in monotonous conditions, with both simulated and on-the-road driving settings, were investigated in Chapters 3 and 4, respectively. Compared to normal sleepers, a performance decrement in PI patients that occurred after 20 minutes of driving was observed in the simulated 
driving task (Chapter 3) which was not found in on-the-road driving (Chapter 4). Our findings for the PVT showed no performance difference between PI patients and good sleepers (Chapter 3). In contrast, the overall RT at the ANT was longer in PI patients compared to good sleepers (Chapter 2). Results from Chapter 2 also revealed no performance difference between groups for each of the three attentional networks (alerting, orienting and executive function). However, compared to good sleepers, PI patients performed more slowly in the incongruent flanker condition (i.e. conflict situation) than in the congruent and neutral flanker conditions, reflecting a conflict resolution deficit.

Two main differences between the simulated and the on-the-road driving task characteristics may explain the discrepant results found between the two driving investigations in PI: 1) driving simulators generally cause higher levels of sleepiness than real driving tasks, and 2) PI patients in the driving simulation studies were generally younger than those in the real driving study.

Contrary to the simulated driving task that utilizes a landscape circuit without any stimulus, the on-the-road driving task requires participants to confront incoming stimuli such as other cars and traffic signs. The on-the-road driving task also included a number of turning points that were not present in the driving simulation. These characteristics (i.e. incoming stimuli and turning points) may have made the on-the-road driving task less monotonous than the simulated driving test. Moreover, it has also been shown that, during night driving, the driving simulator is associated with more lateral variability than on-the-road driving (53). Therefore, the vigilance decrement observed in PI patients was probably highest in the simulated task that helped to highlight differences between PI patients and good sleepers. Since simulators cause higher sleepiness levels than real driving, generalizations from simulators to real driving must be made with great caution. One may argue that the simulated highway driving task was not realistic and that the driving performance decrement shown in simulated conditions may not apply to real driving conditions. Nonetheless, although driving simulator performance is not strictly comparable to performance in real driving conditions, previous studies have shown the validity of simulated driving compared to on-the-road driving $(53,297)$. Despite some limitations with the use of simulators to assess performance, the driving impairment found in PI patients is 
in line with the increased risk of traffic accidents found in epidemiological studies in insomnia patients $(21,176)$.

The different age ranges (24-77 years old in Chapter 3; 52-71 in Chapter 4) of the participants included in the two studies should also be considered to explain the discrepant findings between the two driving investigations. To illustrate this point, a recent study showed that, in a group of insomnia patients older than 55 years old, daytime impairments were most consistently associated with a younger age group (298). Thus, the older insomnia patients included in the on-the-road driving study may therefore have been less sensitive to driving impairment than the younger insomnia patients that were included in the simulator study. Additional investigations aiming to compare behavioral and cortical modifications associated with younger and older insomnia patients are needed to better characterize the development of daytime impairments of PI patients and the eventual compensation mechanisms associated with older insomnia patients. Better knowledge about compensation mechanisms in older insomnia patients could, for example, provide new targets for implementing strategies to improve daytime performance and thus the daily life of insomnia patients. Nevertheless, although methodological issues may have shown limitations, our findings from Chapter 3 indicate that both insomnia patients and clinicians need to be aware of the driving impairments that might occur in monotonous highway situations in insomnia patients. Further on-the-road driving investigations should be conducted in young insomnia patients in order to assess their driving performance in real traffic conditions.

The behavioral results from the simulated driving task (Chapter 3) indicated that PI patients have more difficulty than good sleepers in sustaining vigilance over time. Indeed, PI patients drove as well as good sleepers during the early parts of the simulated driving task. However, after 20 minutes of driving, PI patients had impaired driving performance compared to good sleepers. Our results also revealed that PI patients performed as well as good sleepers in the PVT, whereas they had a longer overall RT than good sleepers during the ANT. Because of its duration (20 minutes) and complexity (complex RT task), the ANT is more challenging than the PVT (10 minutes duration and simple RT task). Consequently, all these results are in line with previous findings that showed 
performance impairment in PI patients compared to good sleepers using challenging or long duration tasks (69-71,142), whereas no performance impairment was found using simple or short duration tasks (74-76). Although the analysis of performance over time for the ANT did not reveal any significant difference between PI patients and good sleepers, a larger sample size may help to find such ToT effects in the ANT in future studies because insomnia patients constitute a heterogeneous population with different subtype characteristics (e.g. reporting of daytime deficits, depression, types of sleep problems) (299).

We postulated that PI patients have difficulty recruiting appropriate cognitive resources that make them more susceptible to vigilance decrement than good sleepers. In order to investigate this assumption, we investigated EEG fluctuations during an on-the-road driving task in older PI patients and older normal sleepers (Chapter 4). Although we did not find any behavioral impairment over time in PI patients compared to normal sleepers in this study, our EEG results revealed a slight increase in the alpha and beta power spectra throughout the driving task in PI patients. The alpha power spectrum is believed to be attenuated in cases of maximal attention (125-128) and increased cognitive demand $(129,130)$ and fluctuations in the beta power spectrum have been associated with changes in vigilance $(131,132)$. Consequently, these results suggest that older insomnia patients seem unable to allocate and adapt their vigilance levels to accomodate cognitive demand (monotony and the duration of a vigilance task). Such difficulty in allocating and adapting cognitive resources may explain the observed vigilance decrements after 20 minutes of driving in PI patients under simulated driving conditions. Additionally, previous reports have shown performance impairments in highly demanding cognitive tasks in PI patients (see meta-analysis (68) and review (67)). However, because the real driving task is not strictly comparable to other tasks previously used in PI patients, additional investigations using a task known to induce performance impairment in PI patients (e.g. the ANT or a monotonous simulated driving task) while recording EEG would be suitable for further understanding a possible failure to recruit adequate cognitive resources in PI.

Results from Chapter 2 revealed conflict resolution (a process that belongs to the executive control) deficit in untreated PI patients. Indeed, when PI 
patients were faced with the incongruent flankers condition (the conflict situation), they reacted more slowly than in the congruent and neutral flankers conditions, which was not the case for the good sleepers. Our results confirm those of Liu and colleagues that showed impaired executive control in PI patients during the ANT (152). However, due to the possible confounding factor between PI and medication in the study of Liu and colleagues, it was not clear whether the observed impaired executive control performance could have been the result of the insomnia disorder per se or of the well known deleterious effects of medication on cognition (146). Since all the PI patients included in our study were free of medication, we showed that PI itself leads to executive dysfunction. Our findings also underlined the sensitivity of the ANT to reveal conflict resolution deficits in PI. Indeed, a previous investigation using the Stroop task (another conflict task) in PI patients (74) did not show any impairment in PI patients. Since one of the key cortical areas of executive functioning is the prefrontal cortex $(300,301)$, our result is thus in line with neuroimaging studies that reported hypoactivation (77) and reduced gray matter and metabolism $(72,88,89)$ in the prefrontal cortex during the waking state in PI patients. The alerting and orienting network showing a lack of performance difference between PI patients and good sleepers is in line with a previous ANT report in PI patients (152). However, our results for the orienting network performance contrast with those of previous investigations that reported sleep-related attentional bias in PI patients $(154,155,157,158)$. As sleep is anxiety producing in PI patients $(80,159)$, it cannot be excluded that such stimuli may have induced stress in participants compared to non personally relevant stimuli, thus affecting performance in the test used. In agreement with this hypothesis, a recent study in PI patients reported longer response latencies in an emotional Stroop task using sleep-related stimuli compared to non-specific treat stimuli (302).

To conclude, our results revealed a driving performance decrement in PI patients, particularly in simulated monotonous driving conditions (Chapter 3) and in challenging tasks such as the ANT (Chapter 2). These performance impairments are probably due to greater difficulties in sustaining vigilant attention in PI patients than in good sleepers. Our EEG results (Chapter 4) support the idea that the vigilance decrement found in PI patients could be the result of a 
failure to allocate and adapt cognitive resources as a function of the task demand. In addition, we showed, for the first time, a conflict resolution deficit in untreated PI patients. All these daytime deficits found in PI patients can impact their daily life performance because vigilance and executive control are implied in everyday behavior (e.g. scanning supermarket shelves, driving, working). These results could explain the complaints of daytime deficits often reported by insomnia patients. Therefore, clinicians and patients should be warned about a possible risk of traffic accidents, at least in the case of long and monotonous driving such as driving on a highway.

\section{Sleep deprivation enhances Time on Task effects: the case of on- the-road driving}

In Chapter 5, we investigated on-the-road driving performance and background EEG fluctuations, as a function of ToT, after a night of sleep deprivation and after a night of normal sleep in healthy volunteers. Our results showed that one night of total sleep deprivation induces a global deterioration of on-the-road driving performance and an increase in the theta and alpha power spectra. Over time, the driving performance gradually decreased and the theta and alpha power spectra increased, after both normal sleep and after sleep deprivation. The ToT effects on driving performance and EEG were more pronounced after sleep deprivation compared to the normal sleep condition.

We found a global deterioration in the driving performance after a night of sleep deprivation compared to the normal sleep condition. This result is in line with previous studies that reported a driving performance decrement after total or partial sleep deprivation and sleep restriction in both driving simulator $(52,54,63,273,274)$ and on-the-road driving settings $(51,64)$. Our EEG results revealed that, globally, one night of sleep deprivation leads to increased alpha and theta power in all brain areas investigated (i.e. frontal, central and parietooccipital). Since laboratory investigations have shown that alpha is sensitive to attentional demands $(130,260)$ and that the theta band is linked to occurrence of a sleepiness state $(30,136,259,262,303)$, our results may reflect both an increase in attentional demand and state of sleepiness after sleep deprivation compared to the normal sleep condition. 
Furthermore, our findings showed that driving performance gradually decreased over time after both a normal night of sleep and after one night of total sleep deprivation. These ToT effects were more pronounced after sleep deprivation. Similar to the progression of the driving performance, the EEG theta and alpha power increased as a function of ToT after both a normal night of sleep and after TSD. The ToT effects were also more pronounced after sleep deprivation for both EEG bands, which means that sleep deprivation exacerbated attentional demand and fatigue due to ToT effects. Increments in theta and alpha EEG bands suggest increased attentional demand and sleepiness and explain why SDLP increased after sleep deprivation. These findings are consistent with earlier works that demonstrated that ToT effects on behavioral performance are exacerbated by sleepiness due to sleep deprivation $(59,62,270)$. Our findings also support observations from epidemiological studies that revealed an increased risk of traffic accidents following sleep deprivation (26-28).

Our results showed that the theta power spectrum increased with ToT after both normal sleep and following TSD, whereas the alpha power spectrum as well as SDLP were relatively constant over time after normal sleep. The latter results are consistent with a previous daytime on-the-road study (65), but differ from investigations using driving simulators $(63,250)$. These studies revealed an increase in the alpha power spectrum and deterioration of driving performance with ToT, even in well-rested conditions $(63,250)$. The discrepancy between some simulator and on-the-road driving studies is probably due to the highly monotonous condition of the simulated driving task that induces greater lateral position variability and more sleepiness than the on-the-road driving task $(53,64)$. These discrepant findings between some simulators and on-the-road driving studies also underline that data collected in driving simulator studies cannot automatically be generalized to real life driving conditions. Altogether, our results (Chapters 4 and 5) and those of Sandberg and colleagues (65) suggest that ToT effects on EEG are different within a real driving setting compared with a simulator setting. Since few studies have evaluated EEG fluctuations over time following sleep deprivation using on-the-road driving, scarce information is available about detectable and reliable measures of driver fatigue and sleepiness in 
real driving conditions. Up to now, previous investigations aimed at assessing driver fatigue and sleepiness have demonstrated that the theta and alpha EEG bands $(226,236,277)$ were reliable measures that could be used as sleepiness indicators in on-board countermeasure devices. Results from Chapter 5 indicate that, in real driving conditions, the theta band seems particularly appropriate for giving information about the sleepiness state of participants, specifically related to ToT effects. Future investigations aiming to develop drowsy or fatigue detection systems adapted to real highway driving conditions should take this information into account.

In Chapters 3, 4 and 5, our results indicate that the ToT effects on driving performance and spectral EEG induced by acute total sleep deprivation differed from those of PI. Our results from Chapter 4 showed that the driving performance decreased gradually throughout the driving task in the sleep deprivation condition. Similarly, we observed an increase in EEG markers of sleepiness and a decrease in EEG markers of arousal. In contrast, results from Chapter 3 showed that the driving performance of PI patients in simulated monotonous conditions decreased after 20 minutes and that the decrement was not gradual as after sleep deprivation. In the on-the-road driving condition, driving performance decreased slightly with ToT in both PI patients and good sleepers, and only slight variations in the EEG power throughout the driving period were found for PI patients (Chapter 4). The slight variations that were found in EEG with ToT in PI may reflect the difficulty in allocating and adapting vigilance levels in PI patients when driving. This result differs from the classic ToT effects induced by sleep deprivation (progressive decline in performance and in vigilance) (59). The difference is not unexpected, as recent models state that PI patients are characterized by hyperarousal from a cognitive, physiological and cortical point of view $(81,304-306)$, while total sleep deprivation leads to increased sleepiness $(63,115,116)$. Therefore, it seems logical that insomnia and sleep deprivation do not strictly lead to the same behavioral and cortical modifications.

To conclude, our results showed that sleep deprivation exacerbated both driving impairment and sleepiness as a function of ToT. The increased difficulties in maintaining the car trajectory during sleep deprivation compared to a normal 
night of sleep may be the results of the cortical modifications associated with sleep deprivation and ToT. In addition, we suggested that the effects of sleep deprivation on driving performance and EEG in the on-the-road driving setting differ from those in the simulated driving setting. We therefore postulate that drowsy driver detection systems should be developed and tested in real highway driving conditions in addition to simulated conditions. Finally, we showed that the effects of ToT led to different types of vigilance decrements during driving in PI and healthy subjects after acute total sleep deprivation. Insomnia patients display more subtle deficits than those elicited by sleep deprivation that can make them less dangerous on the road than acutely sleep deprived persons.

\section{Pathophysiology of primary insomnia}

In order to explore possible explanations of the pathophysiology of PI, we will discuss results from Chapters $2,3,4$ and 6 and link them with neuroimaging results from previous investigations. We aimed to put forward new hypotheses about the pathophysiological mechanisms associated with PI in order to explain behavioral results found in this dissertation.

In Chapter 6, we found specific prefrontal cortical activity in PI patients compared to activity in what we defined as the "other areas" (central, temporal and occipital cortical areas combined). Of note, during NREM sleep, we found lower delta and higher beta power spectra in PI compared to good sleepers in other areas (central, temporal and occipital cortical areas combined) while no group difference was revealed for the prefrontal area. We also found a higher sigma power spectrum in PI compared to good sleepers during stage $3+4$ in the other areas (central, temporal and occipital cortical areas combined) while no group difference was revealed for the prefrontal area. During REM sleep, the only difference between the two groups was found for the prefrontal electrodes, as we observed that PI patients displayed a lower beta1 power spectrum than good sleepers in this cortical area.

Our result from Chapter 6 revealed a lower beta1 power spectrum in REM in the prefrontal area in PI patients compared to good sleepers, whereas we did find a group difference for the beta1 power spectrum in the other areas (central, temporal and occipital cortical areas combined). Given that the beta EEG 
band is related to the level of central arousal (132), the lower beta1 power found in the prefrontal area in PI patients compared to good sleepers during REM sleep can reflect hypoarousal of this cortical area during REM sleep in PI. During REM sleep, in the prefrontal cortex, there is a reactivation (i.e. an increase in the cortical activity) of the so called "anterior paralimbic REM activation area" $(289,290)$, while other parts of the prefrontal cortex remain deactivated $(289,291)$. We can thus argue that the prefrontal hypoactivation during REM sleep in PI patients as reflected by the lower beta1 power could be linked to a failure in the reactivation of the "anterior paralimbic REM activation area", area which includes the amygdala and the anterior cingulate cortex (ACC) (307). Therefore, our spectral EEG results from Chapter 6 suggest a dysfunction in one of these brain regions in PI during sleep. This hypothesis is supported by a PET study that reported metabolism modifications in the ACC during sleep in PI patients compared to good sleepers (89). Although such a hypothesis needs to be further investigated, the ANT results from Chapter 2 provide additional arguments in its favor. The ANT task is a complex reaction time task that assesses the efficiency of the three attentional networks according to the model of Posner (101) (alerting, orienting and executive control). This task is based on the combination of the cuing paradigm developed by Posner (1978), and the Flanker task proposed by Eriksen and Eriksen (1974). It has been reported that the Flanker task produces more interference and leads to greater activation in the ACC than other conflict tasks such as the Stroop task (153). In Chapter 2, we found a conflict resolution deficit in PI patients, whereas a previous study using the Stroop task failed to show any conflict deficit in PI (74). We may suppose that the greater recruitment of the ACC during the ANT compared to the Stroop task allowed better highlighting of the conflict process impairment in PI because there is a dysfunction of the ACC in PI.

Our EEG results from Chapter 6 and behavioral results from Chapter 2 thus support the idea that there could be an ACC dysfunction during sleep and during the waking state in PI. This hypothesis would be in line with fMRI and spectroscopic findings that have revealed larger volume during the waking state (resting state) and increased metabolism during NREM sleep in the ACC in PI patients compared to good sleepers (95). However, to date, the behavioral 
implications of such ACC dysfunction in PI, particularly when it concerrns driving performance, are not clear.

The ACC is part of the Task Positive Network (TPN) which interacts with the Default Mode Network (DMN) to control task performance during visual spatial attention (308) that is mediated by both bottom-up and top-down mechanisms (See (309), for review). The TPN is known to exert top-down regulation of sensory processing by suppressing irrelevant stimuli, and to initiate and maintain a task-level cognitive set (310-312). The DMN is known to be less active during tasks that require attention or goal-orientation than during resting state paradigms (313), and has been suggested to be involved in the control of vigilance (314). Neuroimaging investigations that aimed to understand the pathophysiology of PI also reported cortical alterations in parts of the DMN (313). Since brain structure modifications and functional alterations have been reported in areas related to the TCN and to the DMN in PI patients, it might be possible that the interplay between top-down and bottom-up information is not completely efficient in PI due to altered connectivity within or between these networks. The cortical areas related to the TPN and the DMN are located in the frontal and parietal lobes. Therefore, if the interplay between top down and bottom up information is not completely efficient in PI, we should expect altered connectivity within or between frontal and parietal lobes in PI patients. Indeed, it has been reported disturbed frontosubcortical connectivity in both PI patients $(89,315)$ and rat models of PI (316). Additionally, a recent study suggested that PI patients display an alteration in the functional connectivity between parietal and frontal lobes (317). Such altered functional connectivity within or between parietal and frontal lobes in PI may lead to problems in the interplay between top-down and bottom-up processing especially during challenging tasks, and may explain consistent performance decrements reported in PI in such tasks conditions. However, the investigations described above addressing altered connectivity within or between frontal and parietal lobes were conducted at rest, and such altered connectivity within or between frontal and parietal lobes in PI patients need to be investigated during task performance.

Taken together, our behavioral and EEG results could support the hypothesis of difficulty in the control of the visual guidance of attention in PI. This 
hypothesis would be in line with alteration in functional connectivity previously shown in the literature. However, future appropriate neuroimaging investigations using tasks known to induce a performance decrement in PI (such as a monotonous driving task or the ANT) are needed to confirm this hypothesis and elucidate its neural basis.

\section{How can driving impairment be predicted?}

Chapters 4 and 5 describe two studies that investigated the relationships between driving (SDLP) and the EEG power spectra (theta, alpha and beta) changes. These two studies were conducted in PI patients and after sleep deprivation, respectively. Our results from both studies did not show any relationship between driving and EEG measures.

In Chapter 4 and 5, we expected to find a relationship between SDLP and EEG measures in both experimental situations based on two previous studies that found a link between background EEG and SDLP $(109,231)$. However, our results are in agreement with other papers that failed to report any link between driving and EEG measures (232-235). As the SDLP changes that occurred over time and between conditions (insomnia vs normal sleepers or sleep deprivation vs normal sleep) were different from those occurring for the EEG measures, it would have been difficult to find any relationship between SDLP and background EEG. As an example, in Chapter 4, we found that the driving performance did not differ between normal sleepers and insomnia patients, while we found a significant difference between the two groups on the EEG indices. Also, in Chapter 5, the driving performance fluctuations across ToT were different from those on the EEG, particularly in the sleep deprivation conditions. The lack of correlation found between EEG and SDLP may also be due to the driving performance parameter commonly used in on-the-road driving studies (SDLP). Simulated driving studies that found significant correlation with EEG used other driving performance parameters (driving errors and accidents) $(116,249)$. However, the quantification of such indices in on-the-road driving investigations is not suitable for safety reasons. A recent on-the-road study proposed using lapses of attention (defined as a continuous change in lateral position of greater than $100 \mathrm{~cm}$, lasting for at least 8 seconds) as an outcome measure of driving performance in addition 
to SDLP (318). As lapses of attention are related to sleepiness, future on-the-road driving investigations may be successful in finding significant relationships with background EEG. Taken together, results from the current thesis and those from previous studies lead to the conclusion that EEG may not be ideal for predicting on-the-road driving performance changes as measured with SDLP. However, other outcomes such as lapses of attention could be successfully used for this purpose in future on-the-road driving and EEG investigations.

Other measures besides EEG have been proposed for predicting driving impairment such as the use of electrooculography (EOG) both in simulated and on-the-road driving investigations (65,319-321). However, these studies led to inconsistent results about correlations between EOG and driving performance indices. The simulated driving investigation of Sommer and Golz (320) revealed a linear dependence between percentage of eye closure and SDLP as well as subjective sleepiness (KSS). Similarly, the on-the-road driving study conducted at night by Hallvig and colleagues (319) revealed that long blink duration predicted lane departures. In contrast, the simulated driving study by Shin and colleagues (321) did not report any relationship between EOG and driving performance indices. Laboratory test batteries have also been proposed recently to predict changes in driving performance after alcohol intake, sleep deprivation $(47,267)$ and after psychopharmacological drug intake $(247,322)$. The greater impairments in performance in these experimental situations probably explain that they found a link between laboratory tests measures and driving measures, contrary to our studies where mild impairments were found.

\section{Future research}

Our results underlined a vigilance decrement and executive function deficits in PI patients. However, the implication of specific network connectivity alterations that may explain behavioral deficits in PI patients need to be further investigated. As stated in the introduction, neuroimaging investigations during task performance in PI patients are few and revealed mainly prefrontal modifications $(72,77)$. Considering our results and those from neuroimaging studies, we proposed, among other measures, the ACC and the DMN as new targets for neuroimaging investigations during task performance. As tasks employed should lead to a 
vigilance decrement in PI patients, the driving task seems a good candidate to be used in an MRI study. Such a study would help to further understand the neurocognitive basis of the daytime deficits of PI patients. In addition, the neurobehavioral basis of the conflict resolution deficit found in PI patients should be elucidated using the ANT in the scanner to investigate attentional network related BOLD activity in these patients. A better understanding of the neurobehavioral basis of PI should help to develop new treatments, preferably non pharmacologic, and to enhance daytime performance in these patients.

We have found discrepant results between our simulated and on-the-road driving studies in PI patients. We proposed that the higher levels of sleepiness induced by the simulator compared to real driving partly explained these discrepant findings. Therefore, additional on-the-road and simulated driving studies with the same groups of participants (insomnia patients and good sleepers) are needed in order to explore differences between on-the-road and simulated driving in insomnia and thus determine the driving safety of these patients. We also assumed that old and young PI patients might not have the same daytime deficits in driving performance and that older PI patients might have developed adaptive behavior to confront their sleep disturbances in comparison with younger PI patients. However, as the neurobehavioral mechanisms that could lead to such adaptive behavior in older PI patients have not yet been investigated, it is advisable to conduct additional on-the-road driving investigations that will include both young and old participants. Although complementary investigations should be conducted, the present driving studies described in the current dissertation revealed that PI patients need to be informed about a possible risk of driving impairment in cases of highly monotonous driving conditions.

As the driving impairments described in this dissertation have been found in untreated PI patients, and as most PI patients took hypnotics (323), the combination of PI and hypnotics intake is an important issue in traffic safety. Many studies have previously shown the impairing effects of hypnotics in healthy subjects on driving performance $(180,324,325)$. Previous simulated and on-theroad driving investigations showed that single and repeated bedtime administration of hypnotics impairs highway driving performance of PI patients the next morning $(177,221)$. In contrast, the on-the-road highway driving 
performance of PI patients that chronically or non-chronically use hypnotics was not impaired (221). In order to better understand the adverse or non adverse effects of hypnotics on driving performance in insomnia patients, it could be wise to elucidate the cortical mechanisms associated with chronic and non chronic use of hypnotics in PI patients in relationship to driving performance impairment using EEG. As an example, the use of EEG could underline tolerance mechanisms related to chronic use compared to non chronic use of hypnotics and help to explain how hypnotics can affect driving performance. To our knowledge, only Staner and colleagues (177) have investigated the EEG modifications during driving in PI patients that use hypnotics and showed EEG alterations associated with single and repeated administration of hypnotics. The authors thus suggested that next-day poor driving performance could be related to a prolonged central nervous system effect of hypnotics. Therefore, little is known about the effects of insomnia per se and in combination with treatment with hypnotics on EEG fluctuations during driving. Further concomitant driving and EEG investigations including both treated and untreated insomnia patients as well as good sleepers, are thus needed. This may help to better underline the long-term effectiveness of hypnotic treatment as well as their behavioral and cortical deleterious effects in insomnia patients.

Alternatively, the effectiveness of non-pharmacologic treatments to improve driving performance in insomnia patients should be assessed, for example using Cognitive Behavioral Therapy (CBT). It consists mainly of cognitive restructuring techniques that question sleep paradigms about the lack of sleep and the daytime consequences. In addition, this method aims to reduce or prevent the person from paying excessive attention to sleep or from worrying about not falling asleep by encouraging the patient to intentionally stay awake or to give up all efforts to fall asleep. Although CBT has been shown to be effective for PI, some PI patients did not respond to this treatment (326-329) probably because of interindividual differences (e.g. related to insomnia subtypes, emotional and physical states) (299,329-333). Using CBT, it is expected that vigilance of PI patients will thus probably improve their driving performance. Such improvement should be evaluated both in the short- and long-term, e.g. immediately after CBT and one year later. 


\section{Conclusions}

The research described in this dissertation adressed the effects of PI and sleep deprivation on performance and EEG during both waking and sleep states. Even though we found discrepant results between simulated and on-the-road driving investigations, part of our results revealed a driving performance decrement in PI patients after sleep deprivation. In order to reveal any significant driving performance decrements in PI patients in real driving conditions, additional onthe-road studies are needed with inclusion of young and old participants. As studies described in the current dissertation showed vigilance and conflict resolution deficits in PI, patients and clinicians should be informed about the deleterious impact of PI on daily life activities such as driving. EEG does not seem effective in predicting changes in on-the-road driving performance, and future studies should focus on other measures to do so. This dissertation opened the discussion on new working hypotheses to further investigate the pathophysiology of PI during both the day and night. Future research is needed to fill the gaps remaining in various aspects of the current research. 



\section{References}

1. Bin YS, Marshall NS, Glozier N. Sleeping at the limits: the changing prevalence of short and long sleep durations in 10 countries. Am J Epidemiol. $2013 \mathrm{Apr}$ 15;177(8):826-33.

2. Conti AA. Is our sleep time too short? Sleep deprivation as a pathophysiological condition of modern times. Acta Bio-Medica Atenei Parm. 2012 Dec;83(3):2345.

3. Roth T, Roehrs T. Insomnia: epidemiology, characteristics, and consequences. Clin Cornerstone. 2003;5(3):5-15.

4. Buysse DJ. Insomnia. JAMA J Am Med Assoc. 2013 Feb 20;309(7):706-16.

5. Morin CM, LeBlanc M, Daley M, Gregoire JP, Mérette C. Epidemiology of insomnia: prevalence, self-help treatments, consultations, and determinants of help-seeking behaviors. Sleep Med. 2006 Mar;7(2):123-30.

6. Ohayon MM. Epidemiology of insomnia: what we know and what we still need to learn. Sleep Med Rev. 2002 Apr;6(2):97-111.

7. Ohayon MM, Reynolds CF 3rd. Epidemiological and clinical relevance of insomnia diagnosis algorithms according to the DSM-IV and the International Classification of Sleep Disorders (ICSD). Sleep Med. 2009 Oct;10(9):952-60.

8. Wang Q, Xi B, Liu M, Zhang Y, Fu M. Short sleep duration is associated with hypertension risk among adults: a systematic review and meta-analysis. Hypertens Res Off J Jpn Soc Hypertens. 2012 Oct;35(10):1012-8.

9. Cappuccio FP, D’Elia L, Strazzullo P, Miller MA. Sleep duration and all-cause mortality: a systematic review and meta-analysis of prospective studies. Sleep. 2010 May;33(5):585-92.

10. Baglioni C, Riemann D. Is chronic insomnia a precursor to major depression? Epidemiological and biological findings. Curr Psychiatry Rep. 2012 Oct;14(5):511-8.

11. Daley M, Morin CM, LeBlanc M, Grégoire JP, Savard J, Baillargeon L. Insomnia and its relationship to health-care utilization, work absenteeism, productivity and accidents. Sleep Med. 2009;10(4):427-38. 
12. Fortier-Brochu E, Beaulieu-Bonneau S, Ivers H, Morin CM. Relations between sleep, fatigue, and health-related quality of life in individuals with insomnia. J Psychosom Res. 2010;69(5):475-83.

13. Hamblin JE. Insomnia: an ignored health problem. Prim Care. 2007;34(3):65974 , viii.

14. Hillman DR, Lack LC. Public health implications of sleep loss: the community burden. Med J Aust. 2013 Oct 21;199(8):S7-10.

15. Ozminkowski RJ, Wang S, Walsh JK. The direct and indirect costs of untreated insomnia in adults in the United States. Sleep. 2007;30(3):263-73.

16. Daley M, Morin CM, LeBlanc M, Grégoire J-P, Savard J. The economic burden of insomnia: direct and indirect costs for individuals with insomnia syndrome, insomnia symptoms, and good sleepers. Sleep. 2009;32(1):55-64.

17. Léger D, Bayon V. Societal costs of insomnia. Sleep Med Rev. 2010;14(6):379_ 89.

18. Shahly V, Berglund PA, Coulouvrat C, Fitzgerald T, Hajak G, Roth T, et al. The associations of insomnia with costly workplace accidents and errors: results from the America Insomnia Survey. Arch Gen Psychiatry. 2012 Oct;69(10):1054-63.

19. Laugsand LE, Strand LB, Vatten LJ, Janszky I, Bjørngaard JH. Insomnia symptoms and risk for unintentional fatal injuries- The HUNT study. Sleep. In press;

20. Smolensky MH, Di Milia L, Ohayon MM, Philip P. Sleep disorders, medical conditions, and road accident risk. Accid Anal Prev. 2011;43(2):533-48.

21. Léger D, Bayon V, Ohayon MM, Philip P, Ement P, Metlaine A, et al. Insomnia and accidents: cross-sectional study (EQUINOX) on sleep-related home, work and car accidents in 5293 subjects with insomnia from 10 countries. J Sleep Res. 2014 Apr;23(2):143-52.

22. Maia Q, Grandner MA, Findley J, Gurubhagavatula I. Short and long sleep duration and risk of drowsy driving and the role of subjective sleep insufficiency. Accid Anal Prev. 2013 Oct;59:618-22.

23. Martiniuk ALC, Senserrick T, Lo S, Williamson A, Du W, Grunstein RR, et al. Sleep-deprived young drivers and the risk for crash: the DRIVE prospective cohort study. JAMA Pediatr. 2013 Jul;167(7):647-55. 
24. Sagaspe P, Taillard J, Bayon V, Lagarde E, Moore N, Boussuge J, et al. Sleepiness, near-misses and driving accidents among a representative population of French drivers. J Sleep Res. 2010;19(4):578-84.

25. Stutts JC, Wilkins JW, Scott Osberg J, Vaughn BV. Driver risk factors for sleeprelated crashes. Accid Anal Prev. 2003;35(3):321-31.

26. Di Milia L, Kecklund G. The distribution of sleepiness, sleep and work hours during a long distance morning trip: a comparison between night- and non-night workers. Accid Anal Prev. 2013 Apr;53:17-22.

27. Di Milia L, Rogers NL, Åkerstedt T. Sleepiness, long distance commuting and night work as predictors of driving performance. PloS One. 2012;7(9):e45856.

28. Philip P, Taillard J, Guilleminault C, Quera Salva MA, Bioulac B, Ohayon M. Long distance driving and self-induced sleep deprivation among automobile drivers. Sleep. 1999;22(4):475-80.

29. Alhola P, Polo-Kantola P. Sleep deprivation: Impact on cognitive performance. Neuropsychiatr Dis Treat [Internet]. 2007;3(5). Available from: http://www.ncbi.nlm.nih.gov.gate2.inist.fr/pmc/articles/PMC2656292/

30. Cajochen C, Foy R, Dijk DJ. Frontal predominance of a relative increase in sleep delta and theta EEG activity after sleep loss in humans. Sleep Res Online SRO. 1999;2(3):65-9.

31. Goel N, Rao H, Durmer JS, Dinges DF. Neurocognitive consequences of sleep deprivation. Semin Neurol. 2009;29(4):320-39.

32. Jewett ME, Dijk DJ, Kronauer RE, Dinges DF. Dose-response relationship between sleep duration and human psychomotor vigilance and subjective alertness. Sleep. 1999;22(2):171-9.

33. Lenné MG, Triggs TJ, Redman JR. Interactive effects of sleep deprivation, time of day, and driving experience on a driving task. Sleep. 1998;21(1):38-44.

34. Lim J, Dinges DF. A meta-analysis of the impact of short-term sleep deprivation on cognitive variables. Psychol Bull. 2010 May;136(3):375-89.

35. Bratzke D, Steinborn MB, Rolke B, Ulrich R. Effects of Sleep Loss and Circadian Rhythm on Executive Inhibitory Control in the Stroop and Simon Tasks.

Chronobiol Int. 2012;29(1):55-61. 
36. Hoedlmoser K, Griessenberger H, Fellinger R, Freunberger R, Klimesch W, Gruber W, et al. Event-related activity and phase locking during a psychomotor vigilance task over the course of sleep deprivation. J Sleep Res. 2011;20(3):37785.

37. Roca J, Fuentes LJ, Marotta A, López-Ramón M-F, Castro C, Lupiáñez J, et al. The effects of sleep deprivation on the attentional functions and vigilance. Acta Psychol (Amst). 2012;140(2):164-76.

38. Sadeh A, Dan O, Bar-Haim Y. Online assessment of sustained attention following sleep restriction. Sleep Med. 2011;12(3):257-61.

39. Avolio BJ, Kroeck KG, Panek PE. Individual Differences in InformationProcessing Ability as a Predictor of Motor Vehicle Accidents. Hum Factors J Hum Factors Ergon Soc. 1985 Jan 10;27(5):577-87.

40. Brouwer WH, Ponds RW. Driving competence in older persons. Disabil Rehabil. 1994 Sep;16(3):149-61.

41. Brouwer WH, Withaar FK. Fitness to Drive After Traumatic Brain Injury. Neuropsychol Rehabil. 1997;7(3):177-93.

42. Calhoun VD, Pekar JJ, McGinty VB, Adali T, Watson TD, Pearlson GD. Different activation dynamics in multiple neural systems during simulated driving. Hum Brain Mapp. 2002 Jul;16(3):158-67.

43. Coeckelbergh TRM, Cornelissen FW, Brouwer WH, Kooijman AC. The effect of visual field defects on eye movements and practical fitness to drive. Vision Res. 2002 Mar;42(5):669-77.

44. De Raedt R, Ponjaert-Kristoffersen I. The relationship between cognitive/neuropsychological factors and car driving performance in older adults. J Am Geriatr Soc. 2000 Dec;48(12):1664-8.

45. Lundqvist A. Neuropsychological aspects of driving characteristics. Brain Inj BI. 2001 Nov;15(11):981-94.

46. Ratcliff R, Strayer D. Modeling simple driving tasks with a one-boundary diffusion model. Psychon Bull Rev. 2014 Jun;21(3):577-89.

47. Jongen S, Vuurman E, Ramaekers J, Vermeeren A. Alcohol calibration of tests measuring skills related to car driving. Psychopharmacology (Berl). 2014 Jun;231(12):2435-47. 
48. Pilcher JJ, Band D, Odle-Dusseau HN, Muth ER. Human performance under sustained operations and acute sleep deprivation conditions: toward a model of controlled attention. Aviat Space Environ Med. 2007 May;78(5 Suppl):B15-24.

49. Akerstedt T, Peters B, Anund A, Kecklund G. Impaired alertness and performance driving home from the night shift: a driving simulator study. J Sleep Res. 2005 Mar;14(1):17-20.

50. Anund A, Kecklund G, Kircher A, Tapani A, Akerstedt T. The effects of driving situation on sleepiness indicators after sleep loss: a driving simulator study. Ind Health. 2009;47(4):393-401.

51. Bosker WM, Kuypers KPC, Conen S, Kauert GF, Toennes SW, Skopp G, et al. MDMA (ecstasy) effects on actual driving performance before and after sleep deprivation, as function of dose and concentration in blood and oral fluid. Psychopharmacology (Berl). 2012;222(3):367-76.

52. Filtness AJ, Reyner LA, Horne JA. Driver sleepiness-comparisons between young and older men during a monotonous afternoon simulated drive. Biol Psychol. 2012 Mar;89(3):580-3.

53. Hallvig D, Anund A, Fors C, Kecklund G, Karlsson JG, Wahde M, et al. Sleepy driving on the real road and in the simulator-A comparison. Accid Anal Prev. 2012 Nov 10;50C:44-50.

54. Matthews RW, Ferguson SA, Zhou X, Kosmadopoulos A, Kennaway DJ, Roach GD. Simulated driving under the influence of extended wake, time of day and sleep restriction. Accid Anal Prev. 2012;45 Suppl:55-61.

55. Doran SM, Van Dongen HP, Dinges DF. Sustained attention performance during sleep deprivation: evidence of state instability. Arch Ital Biol. 2001 Apr;139(3):253-67.

56. Sarter M, Gehring WJ, Kozak R. More attention must be paid: the neurobiology of attentional effort. Brain Res Rev. 2006 Aug;51(2):145-60.

57. Sturm W, Willmes K. On the functional neuroanatomy of intrinsic and phasic alertness. NeuroImage. $2001 \mathrm{Jul} ; 14(1 \mathrm{Pt}$ 2):S76-84.

58. Sturm W, de Simone A, Krause BJ, Specht K, Hesselmann V, Radermacher I, et al. Functional anatomy of intrinsic alertness: evidence for a fronto-parietalthalamic-brainstem network in the right hemisphere. Neuropsychologia. 1999 Jun;37(7):797-805. 
59. Warm JS, Parasuraman R, Matthews G. Vigilance requires hard mental work and is stressful. Hum Factors. 2008 Jun;50(3):433-41.

60. Horne JA, Wilkinson S. Chronic sleep reduction: daytime vigilance performance and EEG measures of sleepiness, with particular reference to "practice" effects. Psychophysiology. 1985 Jan;22(1):69-78.

61. Richter S, Marsalek K, Glatz C, Gundel A. Task-dependent differences in subjective fatigue scores. J Sleep Res. 2005 Dec;14(4):393-400.

62. Van Dongen HPA, Belenky G, Krueger JM. A local, bottom-up perspective on sleep deprivation and neurobehavioral performance. Curr Top Med Chem. 2011;11(19):2414-22.

63. Otmani S, Pebayle T, Roge J, Muzet A. Effect of driving duration and partial sleep deprivation on subsequent alertness and performance of car drivers. Physiol Behav. 2005;84(5):715-24.

64. Philip P, Sagaspe P, Taillard J, Valtat C, Moore N, Akerstedt T, et al. Fatigue, sleepiness, and performance in simulated versus real driving conditions. Sleep. 2005;28(12):1511-6.

65. Sandberg D, Anund A, Fors C, Kecklund G, Karlsson JG, Wahde M, et al. The characteristics of sleepiness during real driving at night--a study of driving performance, physiology and subjective experience. Sleep. 2011 Oct;34(10):1317-25.

66. Durmer JS, Dinges DF. Neurocognitive consequences of sleep deprivation. Semin Neurol. 2005;25(1):117-29.

67. Shekleton JA, Rogers NL, Rajaratnam SMW. Searching for the daytime impairments of primary insomnia. Sleep Med Rev. 2010;14(1):47-60.

68. Fortier-Brochu E, Beaulieu-Bonneau S, Ivers H, Morin CM. Insomnia and daytime cognitive performance: A meta-analysis. Sleep Med Rev [Internet]. 2011; Available from: http://www.ncbi.nlm.nih.gov/pubmed/21636297

69. Altena E, Van Der Werf YD, Strijers RLM, Van Someren EJW. Sleep loss affects vigilance: effects of chronic insomnia and sleep therapy. J Sleep Res. 2008;17(3):335-43. 
70. Edinger JD, Means MK, Carney CE, Krystal AD. Psychomotor performance deficits and their relation to prior nights' sleep among individuals with primary insomnia. Sleep. 2008;31(5):599-607.

71. Varkevisser M, Kerkhof GA. Chronic insomnia and performance in a 24-h constant routine study. J Sleep Res. 2005;14(1):49-59.

72. Joo EY, Noh HJ, Kim J-S, Koo DL, Kim D, Hwang KJ, et al. Brain Gray Matter Deficits in Patients with Chronic Primary Insomnia. Sleep. 2013;36(7):9991007.

73. Mendelson WB, Garnett D, Linnoila M. Do insomniacs have impaired daytime functioning? Biol Psychiatry. 1984 Aug;19(8):1261-4.

74. Orff HJ, Drummond SPA, Nowakowski S, Perils ML. Discrepancy between subjective symptomatology and objective neuropsychological performance in insomnia. Sleep. 2007;30(9):1205-11.

75. Schneider-Helmert D. Twenty-four-hour sleep-wake function and personality patterns in chronic insomniacs and healthy controls. Sleep. 1987

Oct;10(5):452-62.

76. Vignola A, Lamoureux C, Bastien CH, Morin CM. Effects of chronic insomnia and use of benzodiazepines on daytime performance in older adults. J Gerontol B Psychol Sci Soc Sci. 2000;55(1):P54-62.

77. Altena E, Van Der Werf YD, Sanz-Arigita EJ, Voorn TA, Rombouts SARB, Kuijer JPA, et al. Prefrontal hypoactivation and recovery in insomnia. Sleep. 2008;31(9):1271-6.

78. Smith EE, Jonides J. Storage and executive processes in the frontal lobes. Science. 1999 Mar 12;283(5408):1657-61.

79. Feige B, Baglioni C, Spiegelhalder K, Hirscher V, Nissen C, Riemann D. The microstructure of sleep in primary insomnia: An overview and extension. Int J Psychophysiol Off J Int Organ Psychophysiol. 2013 Apr 11;

80. Perlis ML, Giles DE, Mendelson WB, Bootzin RR, Wyatt JK. Psychophysiological insomnia: the behavioural model and a neurocognitive perspective. J Sleep Res. 1997;6(3):179-88. 
81. Riemann D, Spiegelhalder K, Feige B, Voderholzer U, Berger M, Perlis M, et al. The hyperarousal model of insomnia: a review of the concept and its evidence. Sleep Med Rev. 2010;14(1):19-31.

82. Wolynczyk-Gmaj D, Szelenberger W. Waking EEG in primary insomnia. Acta Neurobiol Exp (Warsz). 2011;71(3):387-92.

83. Wu YM, Pietrone R, Cashmere JD, Begley A, Miewald JM, Germain A, et al. EEG power during waking and NREM sleep in primary insomnia. J Clin Sleep Med JCSM Off Publ Am Acad Sleep Med. 2013 Oct 15;9(10):1031-7.

84. Krystal AD, Edinger JD, Wohlgemuth WK, Marsh GR. NREM sleep EEG frequency spectral correlates of sleep complaints in primary insomnia subtypes. Sleep. $2002 ; 25(6): 630-40$.

85. Spiegelhalder K, Regen W, Feige B, Holz J, Piosczyk H, Baglioni C, et al. Increased EEG sigma and beta power during NREM sleep in primary insomnia. Biol Psychol. 2012 Dec;91(3):329-33.

86. Merica H, Blois R, Gaillard JM. Spectral characteristics of sleep EEG in chronic insomnia. Eur J Neurosci. 1998;10(5):1826-34.

87. Perlis ML, Merica H, Smith MT, Giles DE. Beta EEG activity and insomnia. Sleep Med Rev. 2001;5(5):365-76.

88. Altena E, Vrenken H, Van Der Werf YD, van den Heuvel OA, Van Someren EJW. Reduced orbitofrontal and parietal gray matter in chronic insomnia: a voxel-based morphometric study. Biol Psychiatry. 2010 Jan 15;67(2):182-5.

89. Nofzinger EA, Buysse DJ, Germain A, Price JC, Miewald JM, Kupfer DJ. Functional neuroimaging evidence for hyperarousal in insomnia. Am J Psychiatry. 2004 Nov;161(11):2126-8.

90. Muzur A, Pace-Schott EF, Hobson JA. The prefrontal cortex in sleep. Trends Cogn Sci. 2002 Nov 1;6(11):475-81.

91. Saper CB, Scammell TE, Lu J. Hypothalamic regulation of sleep and circadian rhythms. Nature. 2005 Oct 27;437(7063):1257-63.

92. Corsi-Cabrera M, Figueredo-Rodríguez P, Del Río-Portilla Y, Sánchez-Romero J, Galán L, Bosch-Bayard J. Enhanced frontoparietal synchronized activation during the wake-sleep transition in patients with primary insomnia. Sleep. 2012;35(4):501-11. 
93. Spiegelhalder K, Regen W, Baglioni C, Riemann D, Winkelman JW.

Neuroimaging Studies in Insomnia. Curr Psychiatry Rep. 2013 Nov 1;15(11):16.

94. Huang Z, Liang P, Jia X, Zhan S, Li N, Ding Y, et al. Abnormal amygdala connectivity in patients with primary insomnia: evidence from resting state fMRI. Eur J Radiol. 2012 Jun;81(6):1288-95.

95. Winkelman JW, Plante DT, Schoerning L, Benson K, Buxton OM, O’Connor SP, et al. Increased Rostral Anterior Cingulate Cortex Volume in Chronic Primary Insomnia. Sleep. 2013;36(7):991-8.

96. Riemann D, Voderholzer U, Spiegelhalder K, Hornyak M, Buysse DJ, Nissen C, et al. Chronic insomnia and MRI-measured hippocampal volumes: a pilot study. Sleep. 2007;30(8):955-8.

97. Neylan TC, Mueller SG, Wang Z, Metzler TJ, Lenoci M, Truran D, et al. Insomnia severity is associated with a decreased volume of the CA3/dentate gyrus hippocampal subfield. Biol Psychiatry. 2010 Sep 1;68(5):494-6.

98. Dinges DF, Powell JW. Microcomputer analyses of performance on a portable, simple visual RT task during sustained operations. Behav Res Methods Instrum Comput. 1985;17(6):652-5.

99. Basner M, Dinges DF. Maximizing sensitivity of the psychomotor vigilance test (PVT) to sleep loss. Sleep. 2011;34(5):581-91.

100. Fan J, McCandliss BD, Sommer T, Raz A, Posner MI. Testing the efficiency and independence of attentional networks. J Cogn Neurosci. 2002;14(3):340-7.

101. Posner MI, Petersen SE. The attention system of the human brain. Annu Rev Neurosci. 1990;13:25-42.

102. Petersen SE, Posner MI. The Attention System of the Human Brain: 20 Years After. Annu Rev Neurosci [Internet]. 2012; Available from: http://www.ncbi.nlm.nih.gov/pubmed/22524787

103. Davies DR, Parasuraman R. The psychology of vigilance. Academic Press; 1982. $306 \mathrm{p}$.

104. Parasuraman R, Warm JS, See JE. Brain systems of vigilance. The attentive brain. Cambridge, MA, US: The MIT Press; 1998. p. 221-56. 
105. Mackworth JF. Performance decrement in vigilance, threshold, and high-speed perceptual motor tasks. Can J Psychol. 1964 Sep;18:209-23.

106. Fan J, McCandliss BD, Fossella J, Flombaum JI, Posner MI. The activation of attentional networks. NeuroImage. 2005;26(2):471-9.

107. Posner MI. Imaging attention networks. NeuroImage. 2012 Jun;61(2):450-6.

108. O’Hanlon JF. Driving performance under the influence of drugs: rationale for, and application of, a new test. Br J Clin Pharmacol. 1984;18 Suppl 1:121S $129 \mathrm{~S}$.

109. Brookhuis KA, de Waard D. The use of psychophysiology to assess driver status. Ergonomics. 1993;36(9):1099-110.

110. Lal SK, Craig A. A critical review of the psychophysiology of driver fatigue. Biol Psychol. 2001 Feb;55(3):173-94.

111. Paus T, Zatorre RJ, Hofle N, Caramanos Z, Gotman J, Petrides M, et al. Timerelated changes in neural systems underlying attention and arousal during the performance of an auditory vigilance task. J Cogn Neurosci. 1997 May;9(3):392408.

112. De Gennaro L, Marzano C, Veniero D, Moroni F, Fratello F, Curcio G, et al. Neurophysiological correlates of sleepiness: a combined TMS and EEG study. NeuroImage. 2007;36(4):1277-87.

113. Santamaria J, Chiappa KH. The EEG of drowsiness in normal adults. J Clin Neurophysiol Off Publ Am Electroencephalogr Soc. 1987 Oct;4(4):327-82.

114. Makeig S, Jung TP. Changes in alertness are a principal component of variance in the EEG spectrum. Neuroreport. 1995 Dec 29;7(1):213-6.

115. Craig A, Tran Y, Wijesuriya N, Nguyen H. Regional brain wave activity changes associated with fatigue. Psychophysiology. 2012;49(4):574-82.

116. Campagne A, Pebayle T, Muzet A. Correlation between driving errors and vigilance level: influence of the driver's age. Physiol Behav. 2004 Jan;80(4):51524.

117. Romei V, Brodbeck V, Michel C, Amedi A, Pascual-Leone A, Thut G. Spontaneous fluctuations in posterior alpha-band EEG activity reflect variability 
in excitability of human visual areas. Cereb Cortex N Y N 1991. 2008 Sep;18(9):2010-8.

118. Sadaghiani S, Scheeringa R, Lehongre K, Morillon B, Giraud A-L, Kleinschmidt A. Intrinsic connectivity networks, alpha oscillations, and tonic alertness: a simultaneous electroencephalography/functional magnetic resonance imaging study. J Neurosci Off J Soc Neurosci. 2010 Jul 28;30(30):10243-50.

119. Worden MS, Foxe JJ, Wang N, Simpson GV. Anticipatory biasing of visuospatial attention indexed by retinotopically specific alpha-band electroencephalography increases over occipital cortex. J Neurosci Off J Soc Neurosci. 2000 Mar 15;20(6):RC63.

120. Palva S, Palva JM. Functional roles of alpha-band phase synchronization in local and large-scale cortical networks. Front Psychol. 2011;2.

121. Akerstedt T, Gillberg M. Subjective and objective sleepiness in the active individual. Int J Neurosci. 1990 May;52(1-2):29-37.

122. Torsvall L, Akerstedt T. Sleepiness on the job: continuously measured EEG changes in train drivers. Electroencephalogr Clin Neurophysiol. 1987 Jun;66(6):502-11.

123. Laufs H, Krakow K, Sterzer P, Eger E, Beyerle A, Salek-Haddadi A, et al. Electroencephalographic signatures of attentional and cognitive default modes in spontaneous brain activity fluctuations at rest. Proc Natl Acad Sci U S A. 2003 Sep 16;100(19):11053-8.

124. Hanslmayr S, Gross J, Klimesch W, Shapiro KL. The role of $\alpha$ oscillations in temporal attention. Brain Res Rev. 2011 Jun 24;67(1-2):331-43.

125. Oken BS, Salinsky MC, Elsas SM. Vigilance, alertness, or sustained attention: physiological basis and measurement. Clin Neurophysiol. 2006 Sep;117(9):1885-901.

126. Pfurtscheller G, Aranibar A. Event-related cortical desynchronization detected by power measurements of scalp EEG. Electroencephalogr Clin Neurophysiol. 1977 Jun;42(6):817-26.

127. Pfurtscheller G, Stancák A Jr, Neuper C. Event-related synchronization (ERS) in the alpha band--an electrophysiological correlate of cortical idling: a review. Int J Psychophysiol Off J Int Organ Psychophysiol. 1996 Nov;24(1-2):39-46. 
128. Schwartz JRL, Roth T. Neurophysiology of sleep and wakefulness: basic science and clinical implications. Curr Neuropharmacol. 2008;6(4):367-78.

129. Pfurtscheller G, Lopes da Silva FH. Event-related EEG/MEG synchronization and desynchronization: basic principles. Clin Neurophysiol Off J Int Fed Clin Neurophysiol. 1999 Nov;110(11):1842-57.

130. Ray WJ, Cole HW. EEG alpha activity reflects attentional demands, and beta activity reflects emotional and cognitive processes. Science. 1985 May $10 ; 228(4700): 750-2$.

131. Belyavin A, Wright NA. Changes in electrical activity of the brain with vigilance. Electroencephalogr Clin Neurophysiol. 1987 Feb;66(2):137-44.

132. Chapotot F, Jouny C, Muzet A, Buguet A, Brandenberger G. High frequency waking EEG: reflection of a slow ultradian rhythm in daytime arousal. Neuroreport. 2000 Jul 14;11(10):2223-7.

133. Foxe JJ, Morie KP, Laud PJ, Rowson MJ, de Bruin EA, Kelly SP. Assessing the effects of caffeine and theanine on the maintenance of vigilance during a sustained attention task. Neuropharmacology. 2012 Jun;62(7):2320-7.

134. Smulders FT, Kenemans JL, Jonkman LM, Kok A. The effects of sleep loss on task performance and the electroencephalogram in young and elderly subjects. Biol Psychol. 1997 Mar 21;45(1-3):217-39.

135. Jagannath M, Balasubramanian V. Assessment of early onset of driver fatigue using multimodal fatigue measures in a static simulator. Appl Ergon. 2014 Jul;45(4):1140-7.

136. Cajochen C, Brunner DP, Kräuchi K, Graw P, Wirz-Justice A. Power density in theta/alpha frequencies of the waking EEG progressively increases during sustained wakefulness. Sleep. 1995 Dec;18(10):890-4.

137. Phipps-Nelson J, Redman JR, Rajaratnam SMW. Temporal profile of prolonged, night-time driving performance: breaks from driving temporarily reduce time-ontask fatigue but not sleepiness. J Sleep Res. 2011 Sep;20(3):404-15.

138. Campbell IG. EEG Recording and Analysis for Sleep Research. Curr Protoc Neurosci Editor Board Jacqueline N Crawley Al. 2009 Oct;CHAPTER:Unit10.2.

139. Achermann P. EEG analysis applied to sleep. Epileptologie. 2009;26:28-33. 
140. Cooley JW, Tukey JW. An algorithm for the machine calculation of complex Fourier series. Math Comput. 1965;19(90):297-301.

141. Maurice M Ohayon DR. Hierarchy of insomnia criteria based on daytime consequences. Sleep Med. 2012;13(1):52-7.

142. Hauri PJ. Cognitive deficits in insomnia patients. Acta Neurol Belg. 1997;97(2):113-7.

143. Sugerman JL, Stern JA, Walsh JK. Daytime alertness in subjective and objective insomnia: some preliminary findings. Biol Psychiatry. 1985;20(7):741-50.

144. Perrier J, Bertran F, Marie S, Couque C, Bulla J, Denise P, et al. Impaired driving performance associated with effect of time duration in patients with primary insomnia. Sleep. 2014 Sep;37(9):1565-73.

145. Eriksen BA, Eriksen CW. Effects of noise letters upon the identification of a target letter in a nonsearch task. Percept Psychophys. 1974 Jan 1;16(1):143-9.

146. Stewart SA. The effects of benzodiazepines on cognition. J Clin Psychiatry. 2005;66 Suppl 2:9-13.

147. Bocca ML, Marie S, Chavoix C. Impaired inhibition after total sleep deprivation using an antisaccade task when controlling for circadian modulation of performance. Physiol Behav. 2014 Jan 30;124:123-8.

148. Horne JA, Ostberg O. A self-assessment questionnaire to determine morningness-eveningness in human circadian rhythms. Int J Chronobiol. 1976;4(2):97-110.

149. Cohen J. Statistical Power Analysis for the Behavioral Sciences. L. Erlbaum Associates; 1988. 594 p.

150. Elliott R. Executive functions and their disorders. Br Med Bull. 2003;65:49-59.

151. Alvarez JA, Emory E. Executive function and the frontal lobes: a meta-analytic review. Neuropsychol Rev. 2006 Mar;16(1):17-42.

152. Liu H, Wang D, Li Y, Li Z, Zhang Y, Lei F, et al. Examination of daytime sleepiness and cognitive performance testing in patients with primary insomnia. PloS One. 2014;9(6). 
153. Fan J, Flombaum JI, McCandliss BD, Thomas KM, Posner MI. Cognitive and brain consequences of conflict. NeuroImage. 2003 Jan;18(1):42-57.

154. MacMahon KMA, Broomfield NM, Espie CA. Attention bias for sleep-related stimuli in primary insomnia and delayed sleep phase syndrome using the dotprobe task. Sleep. 2006;29(11):1420-7.

155. Marchetti LM, Biello SM, Broomfield NM, Macmahon KMA, Espie CA. Who is pre-occupied with sleep? A comparison of attention bias in people with psychophysiological insomnia, delayed sleep phase syndrome and good sleepers using the induced change blindness paradigm. J Sleep Res. 2006 Jun;15(2):21221.

156. Spiegelhalder K, Espie C, Nissen C, Riemann D. Sleep-related attentional bias in patients with primary insomnia compared with sleep experts and healthy controls. J Sleep Res. 2008;17(2):191-6.

157. Spiegelhalder K, Kyle SD, Feige B, Prem M, Nissen C, Espie CA, et al. The impact of sleep-related attentional bias on polysomnographically measured sleep in primary insomnia. Sleep. 2010;33(1):107-12.

158. Woods H, Marchetti LM, Biello SM, Espie CA. The clock as a focus of selective attention in those with primary insomnia: an experimental study using a modified Posner paradigm. Behav Res Ther. 2009 Mar;47(3):231-6.

159. Drake CL, Pillai V, Roth T. Stress and sleep reactivity: a prospective investigation of the stress-diathesis model of insomnia. Sleep. 2014;37(8):1295-304.

160. Bocca ML, Denise P. Residual effects of hypnotics on disengagement of spatial attention. J Psychopharmacol Oxf Engl. 2000;14(4):401-5.

161. Bocca M-L, Denise P. Total sleep deprivation effect on disengagement of spatial attention as assessed by saccadic eye movements. Clin Neurophysiol Off J Int Fed Clin Neurophysiol. 2006 Apr;117(4):894-9.

162. Konrad K, Neufang S, Hanisch C, Fink GR, Herpertz-Dahlmann B. Dysfunctional attentional networks in children with attention deficit/hyperactivity disorder: evidence from an event-related functional magnetic resonance imaging study. Biol Psychiatry. 2006 Apr 1;59(7):643-51.

163. Kratz O, Studer P, Malcherek S, Erbe K, Moll GH, Heinrich H. Attentional processes in children with ADHD: an event-related potential study using the 
attention network test. Int J Psychophysiol Off J Int Organ Psychophysiol. 2011 Aug;81(2):82-90.

164. Johnson KA, Robertson IH, Barry E, Mulligan A, Dáibhis A, Daly M, et al. Impaired conflict resolution and alerting in children with ADHD: evidence from the Attention Network Task (ANT). J Child Psychol Psychiatry. 2008;49(12):1339-47.

165. Martella D, Casagrande M, Lupiáñez J. Alerting, orienting and executive control: the effects of sleep deprivation on attentional networks. Exp Brain Res Exp Hirnforsch Expérimentation Cérébrale. 2011;210(1):81-9.

166. Backhaus J, Junghanns K, Born J, Hohaus K, Faasch F, Hohagen F. Impaired declarative memory consolidation during sleep in patients with primary insomnia: Influence of sleep architecture and nocturnal cortisol release. Biol Psychiatry. 2006;60(12):1324-30.

167. Schneider C, Fulda S, Schulz H. Daytime variation in performance and tiredness/sleepiness ratings in patients with insomnia, narcolepsy, sleep apnea and normal controls. J Sleep Res. 2004;13(4):373-83.

168. Léger D, Stal V, Guilleminault C, Raffray T, Dib M, Paillard M. [Diurnal consequence of insomnia: impact on quality of life]. Rev Neurol (Paris). 2001;157(10):1270-8.

169. Roth T, Ancoli-Israel S. Daytime consequences and correlates of insomnia in the United States: results of the 1991 National Sleep Foundation Survey. II. Sleep. 1999;22 Suppl 2:S354-8.

170. Chan-Chee C, Bayon V, Bloch J, Beck F, Giordanella J-P, Leger D. [Epidemiology of insomnia in France]. Rev Dépidémiologie Santé Publique. 2011 Dec;59(6):409-22.

171. Leger D, Guilleminault C, Dreyfus JP, Delahaye C, Paillard M. Prevalence of insomnia in a survey of 12,778 adults in France. J Sleep Res. 2000 Mar;9(1):3542.

172. Ohayon MM, Lemoine P. [Daytime consequences of insomnia complaints in the French general population]. L’Encéphale. 2004 Jun;30(3):222-7.

173. Ohayon MM, Bader G. Prevalence and correlates of insomnia in the Swedish population aged 19-75 years. Sleep Med. 2010 Dec;11(10):980-6. 
174. Ohayon MM, Sagales T. Prevalence of insomnia and sleep characteristics in the general population of Spain. Sleep Med. 2010 Dec;11(10):1010-8.

175. Roth C, Achermann P, Borbély AA. Frequency and state specific hemispheric asymmetries in the human sleep EEG. Neurosci Lett. 1999;271(3):139-42.

176. Léger D, Massuel M-A, Metlaine A. Professional correlates of insomnia. Sleep. 2006;29(2):171-8.

177. Staner L, Ertlé S, Boeijinga P, Rinaudo G, Arnal MA, Muzet A, et al. Next-day residual effects of hypnotics in DSM-IV primary insomnia: a driving simulator study with simultaneous electroencephalogram monitoring. Psychopharmacology (Berl). 2005;181(4):790-8.

178. Bocca ML, Le Doze F, Etard O, Pottier M, L'Hoste J, Denise P. Residual effect of zolpidem $10 \mathrm{mg}$ and zopiclone $7.5 \mathrm{mg}$ versus flunitrazepam $1 \mathrm{mg}$ and placebo on driving performance and ocular saccades. Psychopharmacology (Berl). $1999 ; 143(4): 373-9$.

179. Meskali M, Berthelon C, Marie S, Denise P, Bocca M-L. Residual effects of hypnotic drugs in aging drivers submitted to simulated accident scenarios: an exploratory study. Psychopharmacology (Berl). 2009;207(3):461-7.

180. Bocca M-L, Marie S, Lelong-Boulouard V, Bertran F, Couque C, Desfemmes T, et al. Zolpidem and zopiclone impair similarly monotonous driving performance after a single nighttime intake in aged subjects. Psychopharmacology (Berl). 2011;214(3):699-706.

181. Gustavsen I, Bramness JG, Skurtveit S, Engeland A, Neutel I, Mørland J. Road traffic accident risk related to prescriptions of the hypnotics zopiclone, zolpidem, flunitrazepam and nitrazepam. Sleep Med. 2008;9(8):818-22.

182. Orriols L, Delorme B, Gadegbeku B, Tricotel A, Contrand B, Laumon B, et al. Prescription medicines and the risk of road traffic crashes: a French registry-based study. PLoS Med [Internet]. 2010;7(11). Available from: http://www.ncbi.nlm.nih.gov/pubmed/21125020

183. Orriols L, Philip P, Moore N, Castot A, Gadegbeku B, Delorme B, et al. Benzodiazepine-like hypnotics and the associated risk of road traffic accidents. Clin Pharmacol Ther. 2011;89(4):595-601.

184. Salvucci DD. Modeling driver behavior in a cognitive architecture. Hum Factors. 2006;48(2):362-80. 
185. Williamson A, Friswell R. Investigating the relative effects of sleep deprivation and time of day on fatigue and performance. Accid Anal Prev. 2011;43(3):690-7.

186. Connor J, Norton R, Ameratunga S, Robinson E, Civil I, Dunn R, et al. Driver sleepiness and risk of serious injury to car occupants: population based case control study. BMJ [Internet]. 2002;324(7346). Available from: http://www.ncbi.nlm.nih.gov/pubmed/12003884

187. Horne JA, Reyner LA. Sleep related vehicle accidents. BMJ. 1995;310(6979):565-7.

188. Pandi-Perumal SR, Verster JC, Kayumov L, Lowe AD, Santana MG, Pires MLN, et al. Sleep disorders, sleepiness and traffic safety: a public health menace. Braz J Med Biol Res Rev Bras Pesqui Médicas E Biológicas Soc Bras Biofísica Al. 2006;39(7):863-71.

189. Philip P, Sagaspe P, Moore N, Taillard J, Charles A, Guilleminault C, et al. Fatigue, sleep restriction and driving performance. Accid Anal Prev. $2005 ; 37(3): 473-8$.

190. Dinges DF, Pack F, Williams K, Gillen KA, Powell JW, Ott GE, et al. Cumulative sleepiness, mood disturbance, and psychomotor vigilance performance decrements during a week of sleep restricted to 4-5 hours per night. Sleep. 1997 Apr;20(4):267-77.

191. Graw P, Kräuchi K, Knoblauch V, Wirz-Justice A, Cajochen C. Circadian and wake-dependent modulation of fastest and slowest reaction times during the psychomotor vigilance task. Physiol Behav. 2004 Feb;80(5):695-701.

192. Jung CM, Ronda JM, Czeisler CA, Wright KP Jr. Comparison of sustained attention assessed by auditory and visual psychomotor vigilance tasks prior to and during sleep deprivation. J Sleep Res. 2011 Jun;20(2):348-55.

193. Wu H, Stone WS, Hsi X, Zhuang J, Huang L, Yin Y, et al. Effects of different sleep restriction protocols on sleep architecture and daytime vigilance in healthy men. Physiol Res Acad Sci Bohemoslov. 2010;59(5):821-9.

194. Bond A, Lader M. The use of analogue scales in rating subjective feelings. Br J Med Psychol. 1974;47(3):211-8.

195. Brookhuis K, De vries G., Prins van wijngaarden P, Veenstra G, Hommes M, Louwerens JW, et al. The effects of increasing doses of meptazinol (100, 200, 
$400 \mathrm{mg}$ ) and glafenine $(200 \mathrm{mg})$ on driving performance. $1985 \mathrm{Apr}$ [cited 2013 May 6]; Available from: http://trid.trb.org/view.aspx?id=1186966

196. Iber C. The AASM manual for the scoring of sleep and associated events: rules, terminology and technical specifications. American Academy of Sleep Medicine; 2007.

197. Espié Stéphane. Vehicle-driven simulator versus traffic-driven simulator: the INRETS approach. Conference presented at: Proceeding of the Driving Simulation; 1999 Jul 7; Paris, France.

198. Verster JC, Roth T. Standard operation procedures for conducting the on-theroad driving test, and measurement of the standard deviation of lateral position (SDLP). Int J Gen Med. 2011;4:359-71.

199. Drummond SPA, Bischoff-Grethe A, Dinges DF, Ayalon L, Mednick SC, Meloy MJ. The neural basis of the psychomotor vigilance task. Sleep. 2005;28(9):1059-68.

200. Cluydts R, De Valck E, Verstraeten E, Theys P. Daytime sleepiness and its evaluation. Sleep Med Rev. 2002 Apr;6(2):83-96.

201. Pinheiro J C, Bates DM. Mixed Effects Models in S and S-Plus. Springer; 2000. $560 \mathrm{p}$.

202. Long JD, Feng D, Cliff N. Ordinal analysis of behavioral data. In: Schinka JA, Velicer WF, editors. Handbook of psychology: Research methods in psychology, Vol 2. Hoboken, NJ, US: John Wiley \& Sons Inc; 2003. p. 635-61.

203. Warrens MJ. On Association Coefficients for $2 \times 2$ Tables and Properties That Do Not Depend on the Marginal Distributions. Psychometrika. 2008 Dec;73(4):777-89.

204. Nakagawa S, Schielzeth H. A general and simple method for obtaining R2 from generalized linear mixed-effects models. Methods Ecol Evol. 2013;4(2):133-42.

205. Verster JC, Pandi-Perumal SR, Ramaekers JG, Gier JJ de. Drugs, Driving and Traffic Safety. Springer; 2009. 564 p.

206. Otmani S, Demazières A, Staner C, Jacob N, Nir T, Zisapel N, et al. Effects of prolonged-release melatonin, zolpidem, and their combination on psychomotor functions, memory recall, and driving skills in healthy middle aged and elderly volunteers. Hum Psychopharmacol. 2008;23(8):693-705. 
207. Daurat A, Sagaspe P, Moták L, Taillard J, Bayssac L, Huet N, et al. Lorazepam impairs highway driving performance more than heavy alcohol consumption. Accid Anal Prev. 2013 Nov;60:31-4.

208. Chee MWL, Tan JC, Zheng H, Parimal S, Weissman DH, Zagorodnov V, et al. Lapsing during Sleep Deprivation Is Associated with Distributed Changes in Brain Activation. J Neurosci. 2008;28(21):5519-28.

209. Shiffrin R, Schneider W, Schneider W, Schneider W. Controlled and automatic human information processing: II. Perceptual learning, automatic attending and a general theory. Psychol Rev. 1977;84:127-90.

210. Baglioni C, Regen W, Teghen A, Spiegelhalder K, Feige B, Nissen C, et al. Sleep changes in the disorder of insomnia: A meta-analysis of polysomnographic studies. Sleep Med Rev. 2013 Jun 25;

211. Fernandez-Mendoza J, Calhoun S, Bixler EO, Pejovic S, Karataraki M, Liao D, et al. Insomnia with objective short sleep duration is associated with deficits in neuropsychological performance: a general population study. Sleep. 2010;33(4):459-65.

212. Kierlin L, Olmstead R, Yokomizo M, Nicassio P, Irwin MR. Diagnostic and Statistical Manual criteria for insomnia related impairment in daytime functioning: polysomnographic correlates in older adults. Sleep Med. 2012 Aug;13(7):95860 .

213. Uehli K, Mehta AJ, Miedinger D, Hug K, Schindler C, Holsboer-Trachsler E, et al. Sleep problems and work injuries: a systematic review and meta-analysis. Sleep Med Rev. 2014 Feb;18(1):61-73.

214. Gieteling EW, Bakker MS, Hoekema A, Maurits NM, Brouwer WH, van der Hoeven JH. Impaired driving simulation in patients with Periodic Limb Movement Disorder and patients with Obstructive Sleep Apnea Syndrome. Sleep Med. 2012 May;13(5):517-23.

215. Philip P, Sagaspe P, Lagarde E, Leger D, Ohayon MM, Bioulac B, et al. Sleep disorders and accidental risk in a large group of regular registered highway drivers. Sleep Med. 2010;11(10):973-9.

216. Komada Y, Asaoka S, Abe T, Inoue Y. Short sleep duration, sleep disorders, and traffic accidents. IATSS Res. 2013 Jul;37(1):1-7. 
217. Laugsand LE, Strand LB, Vatten LJ, Janszky I, Bjørngaard JH. Insomnia Symptoms and Risk for Unintentional Fatal Injuries-The HUNT Study. Sleep. 2014;37(11):1777-86.

218. Léger D, Bayon V, Ohayon MM, Philip P, Ement P, Metlaine A, et al. Insomnia and accidents: cross-sectional study (EQUINOX) on sleep-related home, work and car accidents in 5293 subjects with insomnia from 10 countries. J Sleep Res. 2013 Nov 15;

219. Lucidi F, Mallia L, Violani C, Giustiniani G, Persia L. The contributions of sleeprelated risk factors to diurnal car accidents. Accid Anal Prev. 2013 Mar;51:13540 .

220. Vaz Fragoso CA, Araujo KLB, Van Ness PH, Marottoli RA. Sleep disturbances and adverse driving events in a predominantly male cohort of active older drivers. J Am Geriatr Soc. 2010 Oct;58(10):1878-84.

221. Leufkens TRM, Ramaekers JG, de Weerd AW, Riedel WJ, Vermeeren A. On-theroad driving performance and driving-related skills in older untreated insomnia patients and chronic users of hypnotics. Psychopharmacology (Berl). 2014 Feb 2;

222. Brookhuis KA, de Waard D. The use of psychophysiology to assess driver status. Ergonomics. 1993 Sep;36(9):1099-110.

223. Schmidt EA, Schrauf M, Simon M, Buchner A, Kincses WE. The short-term effect of verbally assessing drivers' state on vigilance indices during monotonous daytime driving. Transp Res Part F Traffic Psychol Behav. 2011 May;14(3):25160.

224. Akerstedt T, Hallvig D, Anund A, Fors C, Schwarz J, Kecklund G. Having to stop driving at night because of dangerous sleepiness--awareness, physiology and behaviour. J Sleep Res. 2013 Aug;22(4):380-8.

225. Kecklund G, Akerstedt T. Sleepiness in long distance truck driving: an ambulatory EEG study of night driving. Ergonomics. 1993 Sep;36(9):1007-17.

226. Papadelis C, Chen Z, Kourtidou-Papadeli C, Bamidis PD, Chouvarda I, Bekiaris E, et al. Monitoring sleepiness with on-board electrophysiological recordings for preventing sleep-deprived traffic accidents. Clin Neurophysiol Off J Int Fed Clin Neurophysiol. 2007 Sep;118(9):1906-22.

227. Horne JA, Baulk SD. Awareness of sleepiness when driving. Psychophysiology. 2004 Jan;41(1):161-5. 
228. Lal SKL, Craig A. Driver fatigue: electroencephalography and psychological assessment. Psychophysiology. 2002 May;39(3):313-21.

229. Association AP, DSM-IV APATF on. Diagnostic and statistical manual of mental disorders: DSM-IV-TR. [Internet]. American Psychiatric Pub; 2000. 996 p. Available from: http://books.google.com/books?id=3SQrtpnHb9MC

230. Cousineau D. Confidence intervals in within-subject designs: A simpler solution to Loftus and Masson's method. Tutor Quant Methods Psychol. 2005;1(1):42-5.

231. Lin C-T, Wu R-C, Liang S-F, Chao W-H, Chen Y-J, Jung T-P. EEG-based drowsiness estimation for safety driving using independent component analysis. IEEE Trans Circuits Syst Regul Pap. 2005 Dec;52(12):2726-38.

232. Simon M, Schmidt EA, Kincses WE, Fritzsche M, Bruns A, Aufmuth C, et al. EEG alpha spindle measures as indicators of driver fatigue under real traffic conditions. Clin Neurophysiol Off J Int Fed Clin Neurophysiol. 2011 Jun;122(6):1168-78.

233. Lowden A, Anund A, Kecklund G, Peters B, Akerstedt T. Wakefulness in young and elderly subjects driving at night in a car simulator. Accid Anal Prev. 2009;41(5):1001-7.

234. Akerstedt T, Ingre M, Kecklund G, Anund A, Sandberg D, Wahde M, et al. Reaction of sleepiness indicators to partial sleep deprivation, time of day and time on task in a driving simulator--the DROWSI project. J Sleep Res. 2010;19(2):298-309.

235. Johnson A, Dawson J, Rizzo M. Lateral control in a driving simulator: correlations with neuropsychological tests and on-road safety errors. Proc Int Driv Symp Hum Factors Driv Assess Train Veh Des. 2011 Dec 1;2011:45-61.

236. Brown T, Johnson R, Milavetz G. Identifying periods of drowsy driving using EEG. Ann Adv Automot Med Annu Sci Conf Assoc Adv Automot Med Assoc Adv Automot Med Sci Conf. 2013;57:99-108.

237. Jap BT, Lal S, Fischer P, Bekiaris E. Using EEG spectral components to assess algorithms for detecting fatigue. Expert Syst Appl. 2009 Mar;36(2, Part 1):2352-9.

238. Zhao C, Zhao M, Liu J, Zheng C. Electroencephalogram and electrocardiograph assessment of mental fatigue in a driving simulator. Accid Anal Prev. 2012 Mar;45:83-90. 
239. Brookhuis K, Louwerens J, O’Hanlon J. EEG energy-density spectra and driving performance under the influence of some antidepressant drugs. Drugs and Driving. Eds. London: Taylor \& Francis; 1986. p. 213-21.

240. Filtness AJ, Reyner LA, Horne JA. Moderate sleep restriction in treated older male OSA participants: greater impairment during monotonous driving compared with controls. Sleep Med. 2011 Oct;12(9):838-43.

241. Verster JC, Taillard J, Sagaspe P, Olivier B, Philip P. Prolonged nocturnal driving can be as dangerous as severe alcohol-impaired driving. J Sleep Res. 2011 Dec;20(4):585-8.

242. Krüger H-P, Kazenwadel J, Vollrath M. Grand Rapids Effects Revisited: Accidents, Alcohol and Risk. 1995.

243. Krüger H-P, Vollrath M. The alcohol-related accident risk in Germany: procedure, methods and results. Accid Anal Prev. 2004 Jan;36(1):125-33.

244. O’Hanlon J. Zopiclone's residual effects on psychomotor and information processing skills involved in complex tasks such as car driving: a critical review. Eur Psychiatry J Assoc Eur Psychiatr. 1995;10 Suppl 3:137s - 43s.

245. O’Hanlon JF, Ramaekers JG. Antihistamine effects on actual driving performance in a standard test: a summary of Dutch experience, 1989-94. Allergy. 1995 Mar;50(3):234-42.

246. Owens K, Ramaekers JG. Drugs, driving, and models to measure driving impairment. In: Verster JC, Pandi-Perumal SR, Ramaekers JG, Gier JJ de, editors. Drugs, Driving and Traffic Safety [Internet]. Birkhäuser Basel; 2009 [cited 2014 Nov 27]. p. 43-58. Available from: http://link.springer.com.gate2.inist.fr/chapter/10.1007/978-3-7643-99238_3

247. Verster JC, Roth T. Predicting psychopharmacological drug effects on actual driving performance (SDLP) from psychometric tests measuring driving-related skills. Psychopharmacology (Berl). 2012;220(2):293-301.

248. Akerstedt T, Anund A, Axelsson J, Kecklund G. Subjective sleepiness is a sensitive indicator of insufficient sleep and impaired waking function. J Sleep Res. 2014 Jun;23(3):240-52. 
249. Eoh HJ, Chung MK, Kim S-H. Electroencephalographic study of drowsiness in simulated driving with sleep deprivation. Int J Ind Ergon. 2005 Apr;35(4):30720.

250. Thiffault P, Bergeron J. Monotony of road environment and driver fatigue: a simulator study. Accid Anal Prev. 2003 May;35(3):381-91.

251. Ramaekers JG, Uiterwijk MM, O’Hanlon JF. Effects of loratadine and cetirizine on actual driving and psychometric test performance, and EEG during driving. Eur J Clin Pharmacol. 1992;42(4):363-9.

252. Vuurman EF, Muntjewerff ND, Uiterwijk MM, van Veggel LM, Crevoisier C, Haglund L, et al. Effects of mefloquine alone and with alcohol on psychomotor and driving performance. Eur J Clin Pharmacol. 1996;50(6):475-82.

253. Lim J, Dinges DF. Sleep Deprivation and Vigilant Attention. Ann N Y Acad Sci. 2008;1129(1):305-22.

254. Hallvig D, Anund A, Fors C, Kecklund G, Karlsson JG, Wahde M, et al. Sleepy driving on the real road and in the simulator--A comparison. Accid Anal Prev. 2013 Jan;50:44-50.

255. Sagaspe P, Taillard J, Akerstedt T, Bayon V, Espié S, Chaumet G, et al. Extended driving impairs nocturnal driving performances. PloS One. 2008;3(10):e3493.

256. Van Dongen HPA, Dinges DF. Sleep, circadian rhythms, and psychomotor vigilance. Clin Sports Med. 2005 Apr;24(2):237-49, vii - viii.

257. Dawson D, Searle AK, Paterson JL. Look before you (s)leep: evaluating the use of fatigue detection technologies within a fatigue risk management system for the road transport industry. Sleep Med Rev. 2014 Apr;18(2):141-52.

258. Achermann P, Dijk DJ, Brunner DP, Borbély AA. A model of human sleep homeostasis based on EEG slow-wave activity: quantitative comparison of data and simulations. Brain Res Bull. 1993;31(1-2):97-113.

259. Aeschbach D, Matthews JR, Postolache TT, Jackson MA, Giesen HA, Wehr TA. Dynamics of the human EEG during prolonged wakefulness: evidence for frequency-specific circadian and homeostatic influences. Neurosci Lett. 1997 Dec 19;239(2-3):121-4.

260. Cooper NR, Croft RJ, Dominey SJJ, Burgess AP, Gruzelier JH. Paradox lost? Exploring the role of alpha oscillations during externally vs. internally directed 
attention and the implications for idling and inhibition hypotheses. Int J Psychophysiol Off J Int Organ Psychophysiol. 2003 Jan;47(1):65-74.

261. Gorgoni M, Ferlazzo F, Ferrara M, Moroni F, D’Atri A, Fanelli S, et al. Topographic electroencephalogram changes associated with psychomotor vigilance task performance after sleep deprivation. Sleep Med. 2014 Sep;15(9):1132-9.

262. Klimesch W. EEG alpha and theta oscillations reflect cognitive and memory performance: a review and analysis. Brain Res Brain Res Rev. 1999 Apr;29(23):169-95.

263. Klimesch W, Schack B, Sauseng P. The functional significance of theta and upper alpha oscillations. Exp Psychol. 2005;52(2):99-108.

264. Oken BS, Salinsky M. Alertness and attention: basic science and electrophysiologic correlates. J Clin Neurophysiol Off Publ Am Electroencephalogr Soc. 1992 Oct;9(4):480-94.

265. Liang SF, Lin CT, Wu RC, Chen YC, Huang TY, Jung TP. Monitoring driver's alertness based on the driving performance estimation and the EEG power spectrum analysis. Conf Proc Annu Int Conf IEEE Eng Med Biol Soc IEEE Eng Med Biol Soc Conf. 2005;6:5738-41.

266. Buysse DJ, Reynolds CF, Monk TH, Berman SR, Kupfer DJ. The Pittsburgh Sleep Quality Index: a new instrument for psychiatric practice and research. Psychiatry Res. 1989;28(2):193-213.

267. Jongen S, Perrier J, Vuurman EF, Ramaekers JG, Vermeeren A. Sensitivity and Validity of Psychometric Tests for Assessing Driving Impairment: Effects of Sleep Deprivation. PloS One. 2015;10(2):e0117045.

268. Mulder-Hajonides van der Meulen W, Wijnberg J, Hollander J, De Diana I, Van den Hoofdakker R. Measurement of subjective sleep quality. Proceedings of the International European Sleep Congress, Elsevier, Amsterdam. 1981.

269. Gratton G, Coles MG, Donchin E. A new method for off-line removal of ocular artifact. Electroencephalogr Clin Neurophysiol. 1983 Apr;55(4):468-84.

270. Asplund CL, Chee MWL. Time-on-task and sleep deprivation effects are evidenced in overlapping brain areas. NeuroImage. 2013 Nov 15;82:326-35. 
271. Freydier C, Berthelon C, Bastien-Toniazzo M, Gineyt G. Divided attention in young drivers under the influence of alcohol. J Safety Res. 2014 Jun;49:13-8.

272. Fischer T, Langner R, Birbaumer N, Brocke B. Arousal and attention: self-chosen stimulation optimizes cortical excitability and minimizes compensatory effort. J Cogn Neurosci. 2008 Aug;20(8):1443-53.

273. Gillberg M, Kecklund G, Akerstedt T. Sleepiness and performance of professional drivers in a truck simulator--comparisons between day and night driving. J Sleep Res. 1996 Mar;5(1):12-5.

274. Jackson ML, Croft RJ, Kennedy GA, Owens K, Howard ME. Cognitive components of simulated driving performance: Sleep loss effects and predictors. Accid Anal Prev [Internet]. 2012; Available from: http://www.ncbi.nlm.nih.gov/pubmed/22721550

275. Strijkstra AM, Beersma DGM, Drayer B, Halbesma N, Daan S. Subjective sleepiness correlates negatively with global alpha $(8-12 \mathrm{~Hz})$ and positively with central frontal theta $(4-8 \mathrm{~Hz})$ frequencies in the human resting awake electroencephalogram. Neurosci Lett. 2003 Apr 3;340(1):17-20.

276. Klimesch W, Sauseng P, Hanslmayr S. EEG alpha oscillations: The inhibitiontiming hypothesis. Brain Res Rev. 2007 Jan;53(1):63-88.

277. Garcés Correa A, Orosco L, Laciar E. Automatic detection of drowsiness in EEG records based on multimodal analysis. Med Eng Phys. 2014 Feb;36(2):244-9.

278. Buysse DJ, Germain A, Hall ML, Moul DE, Nofzinger EA, Begley A, et al. EEG spectral analysis in primary insomnia: NREM period effects and sex differences. Sleep. 2008;31(12):1673-82.

279. Bonnet MH, Arand DL. Hyperarousal and insomnia: state of the science. Sleep Med Rev. 2010;14(1):9-15.

280. Braver TS, Barch DM, Kelley WM, Buckner RL, Cohen NJ, Miezin FM, et al. Direct comparison of prefrontal cortex regions engaged by working and long-term memory tasks. NeuroImage. 2001 Jul;14(1 Pt 1):48-59.

281. Unterrainer JM, Rahm B, Kaller CP, Ruff CC, Spreer J, Krause BJ, et al. When planning fails: individual differences and error-related brain activity in problem solving. Cereb Cortex N Y N 1991. 2004 Dec;14(12):1390-7. 
282. Wilckens KA, Erickson KI, Wheeler ME. Age-Related Decline in Controlled Retrieval: The Role of the PFC and Sleep. Neural Plast. 2012 Aug 28;2012:e624795.

283. Dang-Vu TT, Desseilles M, Laureys S, Degueldre C, Perrin F, Phillips C, et al. Cerebral correlates of delta waves during non-REM sleep revisited. NeuroImage. 2005;28(1):14-21.

284. Dang-Vu TT, Schabus M, Desseilles M, Sterpenich V, Bonjean M, Maquet P. Functional Neuroimaging Insights into the Physiology of Human Sleep. Sleep. 2010 Dec 1;33(12):1589-603.

285. R Core Team. R: A Language and Environment for Statistical Computing [Internet]. Vienna, Austria: R Foundation for Statistical Computing; 2014. Available from: http://www.R-project.org

286. Lakens D. Calculating and reporting effect sizes to facilitate cumulative science: a practical primer for t-tests and ANOVAs. Front Psychol. 2013;4:863.

287. Borbély AA, Mattmann P, Loepfe M, Strauch I, Lehmann D. Effect of benzodiazepine hypnotics on all-night sleep EEG spectra. Hum Neurobiol. 1985;4(3):189-94.

288. Brunner DP, Dijk DJ, Münch M, Borbély AA. Effect of zolpidem on sleep and sleep EEG spectra in healthy young men. Psychopharmacology (Berl). 1991;104(1):1-5.

289. Braun AR, Balkin TJ, Wesenten NJ, Carson RE, Varga M, Baldwin P, et al. Regional cerebral blood flow throughout the sleep-wake cycle. An H2(15)O PET study. Brain J Neurol. 1997 Jul;120 ( Pt 7):1173-97.

290. Nofzinger EA, Mintun MA, Wiseman M, Kupfer DJ, Moore RY. Forebrain activation in REM sleep: an FDG PET study. Brain Res. 1997 Oct 3;770(12):192-201.

291. Maquet P, Péters J, Aerts J, Delfiore G, Degueldre C, Luxen A, et al. Functional neuroanatomy of human rapid-eye-movement sleep and dreaming. Nature. 1996 Sep 12;383(6596):163-6.

292. Bastien CH, St-Jean G, Turcotte I, Morin CM, Lavallée M, Carrier J. Sleep spindles in chronic psychophysiological insomnia. J Psychosom Res. 2009;66(1):59-65. 
293. Besset A, Villemin E, Tafti M, Billiard M. Homeostatic process and sleep spindles in patients with sleep-maintenance insomnia: effect of partial $(21 \mathrm{~h})$ sleep deprivation. Electroencephalogr Clin Neurophysiol. 1998 Aug;107(2):122-32.

294. Le Bon O, Arpi S. [Effect of the first sleep night in polysomnography: classification by variable sensitivity and factorial analysis of differences between nights]. Rev Neurol (Paris). 2003 Nov;159(11 Suppl):6S42-7.

295. Newell J, Mairesse O, Verbanck P, Neu D. Is a one-night stay in the lab really enough to conclude? First-night effect and night-to-night variability in polysomnographic recordings among different clinical population samples. Psychiatry Res. 2012 Dec 30;200(2-3):795-801.

296. Riedel BW, Winfield CF, Lichstein KL. First night effect and reverse first night effect in older adults with primary insomnia: does anxiety play a role? Sleep Med. 2001 Mar;2(2):125-33.

297. Davenne D, Lericollais R, Sagaspe P, Taillard J, Gauthier A, Espié S, et al. Reliability of simulator driving tool for evaluation of sleepiness, fatigue and driving performance. Accid Anal Prev. 2012;45:677-82.

298. Lovato N, Lack L, Wright H, Cant M, Humphreys J. Working memory performance of older adults with insomnia. J Sleep Res. 2013 Jun;22(3):251-7.

299. Benjamins J, Migliorati F, Dekker K, Wassing R, Moens S, Van Someren E, et al. The sleep registry. An international online survey and cognitive test assessment tool and database for multivariate sleep and insomnia phenotyping. Sleep Med. 2013 Dec;14:e293-4.

300. Funahashi S. Neuronal mechanisms of executive control by the prefrontal cortex. Neurosci Res. 2001 Feb;39(2):147-65.

301. Kane MJ, Engle RW. The role of prefrontal cortex in working-memory capacity, executive attention, and general fluid intelligence: an individual-differences perspective. Psychon Bull Rev. 2002;9(4):637-71.

302. Barclay NL, Ellis JG. Sleep-related attentional bias in poor versus good sleepers is independent of affective valence. J Sleep Res. 2013 Aug;22(4):414-21.

303. Hasselmo ME, Eichenbaum H. Hippocampal mechanisms for the contextdependent retrieval of episodes. Neural Netw Off J Int Neural Netw Soc. 2005 Nov;18(9):1172-90. 
304. Bastien CH. Insomnia: Neurophysiological and neuropsychological approaches. Neuropsychol Rev. 2011;21(1):22-40.

305. Buysse DJ, Germain A, Hall M, Monk TH, Nofzinger EA. A Neurobiological Model of Insomnia. Drug Discov Today Dis Models. 2011;8(4):129-37.

306. A.G H. A cognitive model of insomnia. Behav Res Ther. 2002;40(8):869-93.

307. Nofzinger EA. What can neuroimaging findings tell us about sleep disorders? Sleep Med. 2004;5 Suppl 1:S16-22.

308. Wen X, Liu Y, Yao L, Ding M. Top-down regulation of default mode activity in spatial visual attention. J Neurosci Off J Soc Neurosci. 2013 Apr 10;33(15):6444-53.

309. Connor CE, Egeth HE, Yantis S. Visual attention: bottom-up versus top-down. Curr Biol CB. 2004 Oct 5;14(19):R850-2.

310. Dosenbach NUF, Visscher KM, Palmer ED, Miezin FM, Wenger KK, Kang HC, et al. A core system for the implementation of task sets. Neuron. 2006 Jun $1 ; 50(5): 799-812$.

311. Fan J, Gu X, Liu X, Guise KG, Park Y, Martin L, et al. Involvement of the anterior cingulate and frontoinsular cortices in rapid processing of salient facial emotional information. NeuroImage. 2011 Feb 1;54(3):2539-46.

312. Corbetta M, Patel G, Shulman GL. The reorienting system of the human brain: from environment to theory of mind. Neuron. 2008 May 8;58(3):306-24.

313. Raichle ME, MacLeod AM, Snyder AZ, Powers WJ, Gusnard DA, Shulman GL. A default mode of brain function. Proc Natl Acad Sci U S A. 2001 Jan 16;98(2):676-82.

314. Hinds O, Thompson TW, Ghosh S, Yoo JJ, Whitfield-Gabrieli S, Triantafyllou C, et al. Roles of default-mode network and supplementary motor area in human vigilance performance: evidence from real-time fMRI. J Neurophysiol. 2013 Mar;109(5):1250-8.

315. Spiegelhalder K, Regen W, Prem M, Baglioni C, Nissen C, Feige B, et al. Reduced anterior internal capsule white matter integrity in primary insomnia. Hum Brain Mapp. 2014 Jul;35(7):3431-8. 
316. Cano G, Mochizuki T, Saper CB. Neural circuitry of stress-induced insomnia in rats. J Neurosci Off J Soc Neurosci. 2008 Oct 1;28(40):10167-84.

317. Li Y, Wang E, Zhang H, Dou S, Liu L, Tong L, et al. Functional connectivity changes between parietal and prefrontal cortices in primary insomnia patients: evidence from resting-state fMRI. Eur J Med Res. 2014;19.

318. Verster JC, Bervoets AC, de Klerk S, Roth T. Lapses of attention as outcome measure of the on-the-road driving test. Psychopharmacology (Berl). 2014 Jan;231(1):283-92.

319. Hallvig D, Anund A, Fors C, Kecklund G, Akerstedt T. Real driving at night Predicting lane departures from physiological and subjective sleepiness. Biol Psychol. 2014 Sep;101:18-23.

320. Sommer D, Golz M. Evaluation of PERCLOS based current fatigue monitoring technologies. Conf Proc Annu Int Conf IEEE Eng Med Biol Soc IEEE Eng Med Biol Soc Annu Conf. 2010;2010:4456-9.

321. Shin D, Sakai H, Uchiyama Y. Slow eye movement detection can prevent sleeprelated accidents effectively in a simulated driving task. J Sleep Res. 2011 Sep;20(3):416-24.

322. Ramaekers JG. Antidepressants and driver impairment: empirical evidence from a standard on-the-road test. J Clin Psychiatry. 2003 Jan;64(1):20-9.

323. Holbrook AM, Crowther R, Lotter A, Cheng C, King D. Meta-analysis of benzodiazepine use in the treatment of insomnia. CMAJ Can Med Assoc J J Assoc Medicale Can. 2000;162(2):225-33.

324. Leufkens TRM, Vermeeren A. Highway driving in the elderly the morning after bedtime use of hypnotics: a comparison between temazepam $20 \mathrm{mg}$, zopiclone $7.5 \mathrm{mg}$, and placebo. J Clin Psychopharmacol. 2009 Oct;29(5):432-8.

325. Leufkens TRM, Ramaekers JG, de Weerd AW, Riedel WJ, Vermeeren A. Residual effects of zopiclone $7.5 \mathrm{mg}$ on highway driving performance in insomnia patients and healthy controls: a placebo controlled crossover study. Psychopharmacology (Berl). 2014 Jul;231(14):2785-98.

326. National Institutes of Health. National Institutes of Health State of the Science Conference statement on Manifestations and Management of Chronic Insomnia in Adults, June 13-15, 2005. Sleep. 2005 Sep;28(9):1049-57. 
327. Morin CM, Culbert JP, Schwartz SM. Nonpharmacological interventions for insomnia: a meta-analysis of treatment efficacy. Am J Psychiatry. 1994 Aug;151(8):1172-80.

328. Murtagh DR, Greenwood KM. Identifying effective psychological treatments for insomnia: a meta-analysis. J Consult Clin Psychol. 1995 Feb;63(1):79-89.

329. Wang M-Y, Wang S-Y, Tsai P-S. Cognitive behavioural therapy for primary insomnia: a systematic review. J Adv Nurs. 2005 Jun;50(5):553-64.

330. Gehrman PR, Pfeiffenberger C, Byrne E. The Role of Genes in the Insomnia Phenotype. Sleep Med Clin. 2013 Sep 1;8(3):323-31.

331. Harvey C-J, Gehrman P, Espie CA. Who is predisposed to insomnia: a review of familial aggregation, stress-reactivity, personality and coping style. Sleep Med Rev. 2014 Jun;18(3):237-47.

332. Espie CA, Kyle SD, Hames P, Cyhlarova E, Benzeval M. The daytime impact of DSM-5 insomnia disorder: comparative analysis of insomnia subtypes from the Great British Sleep Survey. J Clin Psychiatry. 2012 Dec;73(12):e1478-84.

333. Espie CA, Barrie LM, Forgan GS. Comparative Investigation of the Psychophysiologic and Idiopathic Insomnia Disorder Phenotypes: Psychologic Characteristics, Patients' Perspectives, and Implications for Clinical Management. Sleep. 2012 Mar 1;35(3):385-93. 


\section{Summary}

Sleep disruption is increasingly becoming a major health problem with a number of direct and indirect burdens on our modern societies. It has been shown that sleep deprivation exerts a number of adverse effects on cognitive and driving performance as revealed by epidemiological and experimental investigations. While driving simulator investigations after sleep deprivation demonstrated a performance decrement, real driving investigations in conditions of sleep deprivation are scarce. Moreover, little is known about the EEG fluctuations across time for tasks carried out after sleep deprivation in on-the-road highway driving conditions. In contrast, with sleep deprivation, the link between primary insomnia and daytime performance decrements is still unclear, particularly related to monotonous driving. In addition, executive functioning has rarely been investigated in primary insomnia although these patients complain of difficulties related to this cognitive field. Discrepancy between previous studies raises the question of the sensitivity of the tests used to evaluate executive functioning in primary insomnia. Finally, the EEG modifications associated with primary insomnia during both daytime and nighttime need to be investigated further in order to better elucidate the pathophysiology of this sleep disorder.

This dissertation aimed to identify the daytime performance deficits and EEG modifications associated with sleep deprivation and primary insomnia. In all the experimental studies described in this dissertation, we compared two groups. First, we compared the performances and the EEG fluctuations of primary insomnia patients compared to good sleepers. Second, we compared performances and EEG fluctuations in healthy participants after total sleep deprivation compared to a normal night of sleep. The first three chapters after the introduction focused on daytime performances and daytime EEG fluctuations in primary insomnia patients. The following chapter focused on the effects of sleep deprivation on daytime driving performances and EEG fluctuations compared to a normal night of sleep. The chapter preceding the discussion was dedicated to the spectral modifications of sleep associated with primary insomnia.

Chapter 1 gave a brief overview of current knowledge about insomnia and sleep deprivation. We first presented epidemiological information related to costs 
and prevalence of both insomnia and sleep deprivation. Then, we did a short review of the daytime deficits associated with insomnia and sleep deprivation. We also focused on the previous neuroimaging studies that investigated the pathophysiology of primary insomnia both during the wake state and during sleep. Finally, we described our methods and their usefulness in the context of this PhD.

The aim of the study described in Chapter 2 was to to better characterize cognitive deficits of primary insomnia patients in order to further understand their cognitive complaints. We looked at attentional and executive function because of their high involvement in daily living tasks. For this purpose, twenty-one primary insomnia patients and sixteen good sleepers completed the Attentional Networks Test (ANT). We only included untreated primary insomnia patients since sleep medication could be a confounding factor when assessing cognition. While PI patients, as compared to good sleepers, were found to have a longer overall reaction time and to perform more slowly in the incongruent flankers condition (conflict situation) than in the congruent condition, main effects were not observed for the variables representing the three attentional networks (alerting, orienting, and executive function). The present study revealed a conflict resolution deficit in untreated primary insomnia patients. This impairment may be linked to the prefrontal alterations reported in neuroimaging studies in these patients. Patients also had impaired vigilance compared to good sleepers, likely due to the high cognitive load of the ANT. These results could serve to explain the complaints of primary insomnia patients about difficulties performing daily living tasks that are often cognitively demanding.

In Chapter 3, we aimed to study the driving performance and the psychomotor vigilance of primary insomnia patients compared to good sleepers. After one night of polysomnography, twenty-one primary insomnia patients and sixteen good sleepers performed a 1-h simulated monotonous driving task and a psychomotor vigilance task (PVT). The driving performance was quantified using the SDLP. Self-ratings of sleepiness, mood and driving performance were completed. Results revealed a larger SDLP and more lane crossings in primary insomnia patients than in good sleepers. Analyses of effect of time on task on performance showed that the impairment in patients occurred after twenty minutes of driving, which was not the case for good sleepers. No difference 
between groups was found for the PVT, neither for the mean reaction time (RT) nor the number of lapses and the mean slowest $10 \% 1 /$ RT. Patients rated their sleepiness level higher and their alertness level lower than did good sleepers. The self-evaluation of the driving performance was not different between groups. These findings revealed that primary insomnia is associated with a performance decrement during a simulated monotonous driving task. We also showed that patients are able to drive safely only for a short time. In addition, insomnia patients did not seem aware of their performance decrement although they felt less alert and sleepier than good sleepers.

In Chapter 4, we set out to quantify driving performance and EEG fluctuations across a real driving task in primary insomnia patients and in good sleepers. A second aim was to determine whether changes in spectral EEG measures are related to changes in driving performance in older insomnia patients. For this purpose, global and changes over time for driving performance (SDLP) and background EEG in alpha, theta and beta bands were measured in older, untreated insomnia patients and normal sleepers during a 1-hour on-the-road driving task. Overall SDLP did not differ between groups and increased over time to similar degrees in both groups. Alpha and beta power were generally lower in insomnia patients compared to normal sleepers. Alpha and beta power were slightly reduced during driving in normal sleepers, but remained at a constant low in insomnia patients. Changes in EEG power and SDLP were not related. It was concluded that on-the-road driving performance does not differ between older insomnia patients and older normal sleepers and that changes in spectral EEG measures of cortical arousal and in driving performance were not related. In addition, our EEG results may suggest troubles in adapting vigilance and attention levels across the monotonous real driving task in insomnia patients.

In Chapter 5, the main objective was to assess whether sleepiness exacerbates Time on Task effects on both EEG and driving performance in real driving conditions. We also wanted to know which EEG fluctuations are detectable after sleep deprivation during on-the-road driving. For this purpose, twenty-four participants performed a one-hour monotonous on-the-road driving task both after a normal night of sleep and after one night of sleep deprivation. Continuous EEG was recorded during the on-the-road driving task. We measured 
the SDLP as a measure of road tracking error and spectral power was obtained over three bilateral clusters (frontal, central, parieto-occipital) in the theta, alpha and beta bands. Both datasets were averaged every 10 minutes to evaluate Time on Task effects. Sixteen participants completed the driving task in both sleep conditions (completers). Seven drives were terminated prematurely after one night of sleep deprivation (non-completers). We found effects of sleep deprivation, Time on Task and their interaction, both for SDLP and theta and alpha EEG bands. SDLP and EEG indices increased after sleep deprivation and varied with Time on Task. The latter was more pronounced after sleep deprivation. Beta power spectra increased with Time on Task only. Compared to completers, the SDLP for non-completers tended to be higher. The beta power spectrum varied differently with Time on Task between groups in the Frontal cluster. Changes in SDLP and EEG did not correlate significantly. It was concluded that sleep deprivation exacerbates driving impairment and sleepiness as a function of Time on Task during on-the-road driving. Our results also suggested that participants who completed both driving sessions put more effort across the task compared to those who prematurely terminated the drives.

The investigation described in Chapter 6 was dedicated to assessing the specific prefrontal activity in comparison to those in the other main cortical areas in primary insomnia patients and in good sleepers. For this purpose, participants completed a night of polysomnography. Fourteen primary insomnia patients and eleven good sleepers were included in the analysis after artefact rejection. Power spectra were calculated during NREM (Non rapid eye movements) and REM (Rapid eye movements) sleep at prefrontal, occipital, temporal and central electrode positions. We compared the EEG activity in the theta, alpha and beta bands of the prefrontal cortex compared to other cortical areas. During NREM sleep, the power spectra did not differ between groups in the prefrontal cortex, while primary insomnia patients exhibited a higher beta power spectrum and a lower delta power spectrum compared to good sleepers in other areas. During REM sleep, the betal power spectrum was lower in the prefrontal cortex in primary insomnia patients compared to good sleepers while no significant difference between groups was obtained for the other areas. The study described in this chapter showed a specific prefrontal sleep pattern during the whole sleep 
period. In addition, we suggested that primary insomnia patients displayed a dysfunction in the reactivation of the limbic system during REM sleep and we provided additional arguments in favor of a sleep-protection mechanism displayed by primary insomnia patients.

Finally, Chapter 7 discussed all studies from this dissertation and drew some general conclusions about the daytime performance associated with sleep deprivation and primary insomnia with a special focus on time on task effects during driving. In addition, new hypotheses were put forward about the pathophysiology of primary insomnia in light of the EEG and behavioral results described in the current dissertation. At the end, we discussed the topic of the prediction of driving performance changes using EEG and proposed other approaches to achieve this goal. Finally, future research questions were raised related to daytime driving performance in primary insomnia patients. Overall, this dissertation revealed that primary insomnia is associated with a vigilance decrement that could have adverse effects on monotonous driving under specific conditions. In addition, executive functioning seemed to be affected in a subtle way in primary insomnia. Future imaging investigations in this field are needed. In addition, the specific sleep pattern revealed in insomnia patients was further discussed. We also showed that sleep deprivation enhanced time on task effects on real driving performance which raises the question of this cumulative effect on traffic safety in everyday life. In summary, we demonstrated a better understanding of the adverse daytime consequences of primary insomnia and total sleep deprivation with a time on task aspect, and we furthered a new hypothesis about the pathophysiology of primary insomnia. 



\section{Résumé}

Les perturbations de la qualité et de la quantité du sommeil constituent un problème de santé publique croissant dans nos sociétés modernes avec des conséquences économiques importantes. Des études épidémiologiques et expérimentales ont montré que la privation de sommeil avait des effets délétères sur les performances cognitives et de conduite automobile. En particulier, des études en conduite simulée ont systématiquement révélé des détériorations de la performance de conduite. Contrairement aux études en conduite automobile simulée, les études sur les effets de la privation de sommeil dans un contexte de conduite réelle sont peu nombreuses. De plus, très peu d'informations sont disponibles quant aux effets de la privation de sommeil sur les fluctuations du signal EEG et de la performance au cours d'une tâche de conduite en condition réelle. Les performances diurnes des insomniaques primaires sont, en revanche, moins bien comprises particulièrement au regard de la conduite automobile. De plus, les performances dans le domaine des fonctions exécutives ont été très peu étudiées chez les insomniaques primaires bien que ces patients reportent fréquemment des difficultés en lien avec ce domaine cognitif. Les quelques études dans le domaine n'ont pas pu révéler de déficits exécutifs, ce qui peut soulever la question de la sensibilité des tests utilisés classiquement dans l'évaluation des fonctions exécutives. Enfin, l'étude des fluctuations du signal EEG, que ce soit au cours de tâches diurnes ou durant le sommeil, permettrait de mieux comprendre la pathophysiologie de l'insomnie et ses mécanismes.

Cette thèse visait à mieux caractériser les déficits diurnes ainsi que les fluctuations du signal EEG dans l'insomnie primaire et en condition de privation de sommeil. Dans ce but, les études expérimentales décrites dans le cadre de ce travail ont comparé de façon systématique deux groupes de participants. D’une part, nous avons comparés les performances diurnes et les modifications du signal EEG de patients insomniaques par rapport à des bons dormeurs. D'autres parts, nous avons comparés les performances diurnes et les modifications du signal EEG chez des participants sains après privation totale de sommeil par rapport à une nuit normale de sommeil. Cinq chapitres experimentaux sont décrits dans cette dissertation. Dans les trois premiers chapitres expérimentaux de cette 
dissertation, nous nous sommes intéressés aux effets de l'insomnie primaire sur les performances diurnes et sur les fluctuations du signal EEG lors de la conduite automobile. Dans le quatrième chapitre nous avons porté notre attention sur les effets d'une privation totale de sommeil sur les performances de conduite et sur les fluctuations du signal EEG en condition de conduite réelle. Enfin, le dernier chapitre expérimental de cette thèse est dédié à l'étude des modifications spectrales du signal EEG au cours du sommeil des insomniaques primaires.

Le Chapitre 1 nous a permis de dresser un bref état des lieux de la littérature sur les thématiques de l'insomnie et de la privation de sommeil. Nous avons d'abord abordé les études épidémiologiques qui ont mis en avant la prévalence et les couts associés à l'insomnie et à la privation de sommeil. Nous avons ensuite fait une courte revue de littérature à propos des déficits diurnes associés à l'insomnie et à la privation de sommeil. Nous avons également décrits les précédentes études qui se sont attachées à comprendre la pathophysiologie de l'insomnie primaire en utilisant la neuroimagerie tant durant la veille que durant le sommeil. Enfin, nous avons décrit les différents méthods et matériels employés dans cette thèse ainsi que leur utilité au regard des questions posées.

L'objectif de l'étude décrite dans le Chapitre $\mathbf{2}$ était de caractériser les déficits cognitifs des patients souffrant $\mathrm{d}^{\prime}$ insomnie primaire afin de mieux comprendre leurs plaintes cognitives. Nous avons examiné les fonctions attentionnelles et exécutives en raison de leur forte implication dans les tâches de la vie quotidienne. À cet effet, vingt et un patients insomniaques primaires et seize bons dormeurs ont effectué une tâche attentionnelle (Attentional Networks Test ANT). Seuls des patients non traités ont été inclus puisque la consommation de somnifères peut être un facteur confondant lors de l'évaluation cognitive. Les patients insomniaques étaient plus lents à réagir que les bons dormeurs en cas de situations de conflit (cibles non congruentes). Le temps de réaction global au test d'ANT était également plus long chez les patients par rapport aux bons dormeurs. En revanche, aucune différence entre les groupes n'a été observée pour chacun des trois réseaux attentionnels évalués par l’ANT (l’alerte, l'orientation, et les fonctions exécutives). Notre étude a donc permis de révéler un problème de résolution de conflit chez les patients insomniaques non traités. Cette difficulté en cas de situation de conflit pourrait être liée à des modifications préfrontales 
rapportées dans les études de neuro-imagerie. Les patients avaient également un déficit de vigilance par rapport aux bons dormeurs, probablement en raison de la forte charge cognitive de l'ANT. Ces résultats permettent de mettre en lumière les déficits cognitifs dont se plaignent les patients insomniaques, notamment en rapport avec des difficultés à accomplir des tâches à la vie quotidienne qui sont souvent exigeantes en terme cognitif (ex : planification).

Dans le Chapitre 3, nous nous sommes attachés à étudier la performance de conduite et le niveau de vigilance de patients souffrant d'insomnie primaire par rapport à des bons dormeurs. Après une nuit d'enregistrement du sommeil, vingt et un patients insomniaques et seize bons dormeurs ont effectué une tâche de conduite monotone simulée pendant une heure et une tâche évaluant le niveau de vigilance (Psychomotor Vigilance Test - PVT). La performance de conduite a été quantifiée par l'écart type de la position latérale (Standard deviation of Lateral Position - SDLP). Les participants ont également rempli des questionnaires d'auto-évaluation de la somnolence, de l'humeur, et des performances de conduite. Les résultats ont révélé que l’écart type de la position latérale est plus important et que les sorties de route sont plus nombreuses chez les patients insomniaques que chez les bons dormeurs. L'analyse des effets temporels sur la performance de conduite a montré que la détérioration de la performance de conduite chez les insomniaques se produisait vingt minutes après le début de la tâche ce qui n’était pas le cas pour les bons dormeurs. Aucune différence entre les groupes n’a été montrée pour le PVT. Les patients ont évalué leur niveau de somnolence supérieur et leur niveau de vigilance inférieur à celui des bons dormeurs. L'auto-évaluation de la performance de conduite n'a pas montré de différence entre les groupes. Cette étude a donc mis en évidence une détérioration de la performance de conduite simulée chez des insomniaques primaires. En particulier, les patients semblent en mesure de conduire en toute sécurité seulement pendant un court laps de temps avant que leur performance ne se dégrade. En outre, les patients insomniaques ne semblent pas conscients de la détérioration de leur performance de conduite malgré le fait qu’ils se soient décrits comme moins alertes et plus somnolents que les bons dormeurs.

Le Chapitre 4 était dédié à l'étude des fluctuations de la performance de conduite et du signal EEG au cours d'une tâche de conduite réelle chez des 
patients insomniaques par rapport à des bons dormeurs. Nous voulions également savoir si les fluctuations du signal EEG et de la performance de conduite étaient liées chez les insomniaques. A cet effet, nous avons quantifié les modifications globales au cours du temps, de la performance de conduite (mesurée par l'écart type de la position latérale - SDLP) et du signal EEG dans les bandes de fréquence alpha, thêta et bêta lors d'une tâche de conduite réelle d'une durée d'une heure chez des patients insomniaques et chez des bons dormeurs âgés de plus de 55 ans. Globalement, l'écart type de la position latérale ne différait pas entre les groupes et augmentait au cours du temps de façon similaire dans les deux groupes. La puissance spectrale dans les bandes de fréquence alpha et bêta était globalement plus faible chez les patients par rapport aux bons dormeurs. Au cours du temps, la puissance spectrale dans ces deux bandes de fréquences diminuait légèrement chez les bons dormeurs alors qu'elle était relativement constante chez les patients insomniaques. Nous n'avons pas démontré de relation entre les variations du signal EEG et les variations de la performance de conduite. Nous avons conclu que les performances de conduite en condition réelle ne différaient pas entre les patients insomniaques et les bons dormeurs. Et que les fluctuations de la performance de conduite n’étaient pas liées à celles du signal EEG. Nos résultats EEG peuvent également suggérer une difficulté de recrutement des ressources cognitives nécessaires à la réalisation de la tâche de conduite chez les patients souffrant d'insomnie primaire.

Dans le Chapitre 5, l'objectif principal était d'évaluer si la privation de sommeil aggravait les effets délétères de la durée de la tâche (effets Time on Task) à la fois sur le signal EEG et sur les performances de conduite en condition de conduite réelle. Un deuxième objectif était de savoir quelles étaient les modifications du signal EEG détectables après une nuit de privation de sommeil lors de la conduite automobile en condition réelle. A cet effet, vingt-quatre participants ont effectué une tâche de conduite réelle d'une heure après une nuit normale de sommeil et après une nuit de privation de sommeil. Le signal EEG a été enregistré en continue durant la tâche de conduite. Nous avons mesuré l'écart type de la position latérale (SDLP) comme mesure de la performance de conduite et nous avons quantifié la puissance spectrale dans les bandes de fréquences thêta, alpha et beta au niveau de trois zones corticales bilatérales (frontale, centrale, 
pariéto-occipitale). Pour l'ensemble des données, la moyenne du signal a été obtenue toutes les 10 minutes afin de quantifier l'effet de la durée de la tâche (Time on Task). Seize participants ont terminé la tâche de conduite dans les deux conditions (avec ou sans privation de sommeil) ; en revanche, les sept autres n’ont pas pu terminer la tâche de conduite lors de la condition de privation de sommeil. Nos résultats ont montré un effet de la privation de sommeil, un effet de la durée de la tâche ainsi qu'une interaction entre les deux aussi bien sur l'écart type de la position latérale que sur les puissances spectrales EEG dans les bandes thêta et alpha. Les effets temporels sur la performance de conduite et sur le signal EEG étaient plus prononcés après privation de sommeil par rapport à la condition contrôle. La puissance spectrale dans la bande beta était augmentée au cours de la tâche de conduite mais aucun effet significatif de la privation de sommeil n'a été trouvé. En comparaison aux participants qui ont terminé la tâche de conduite dans les deux conditions, ceux qui n’ont pas terminé la tâche en condition de privation de sommeil avaient un écart type de la position latérale plus élevé. La puissance spectrale dans la bande de fréquence beta au niveau frontal variait différemment au cours du temps entre les deux groupes. Les modifications de la performance de conduite et du signal EEG n’étaient pas corrélées de manière significative. Nous avons conclu que la somnolence induite par la privation de sommeil aggravait les effets de la durée de la tâche lors de la conduite réelle. Nos résultats suggèrent également que les participants qui ont terminé les deux sessions de conduite (avec ou sans privation de sommeil) ont probablement mis plus d'effort dans la réalisation de la tâche que ceux qui ont terminé la tâche de conduite prématurément en condition de privation de sommeil.

L'étude décrite dans le Chapitre 6 a été consacrée à la quantification de l'activité corticale au niveau du cortex préfrontal et au niveau des autres principales aires corticales chez des patients insomniaques primaires et chez des bons dormeurs. Dans ce but, les participants ont effectué une nuit d'enregistrement du sommeil et, à la suite du rejet d'artefacts, quatorze patients et onze bons dormeurs ont été inclus dans l'analyse spectrale du sommeil. Les puissances spectrales ont été obtenues pendant le sommeil lent profond (nonrapid eye movements - NREM) et le sommeil paradoxal (rapid eye movements REM) au niveau des électrodes préfrontales, occipitales, temporales et centrales. 
Nous avons comparé la puissance spectrale dans les bandes de fréquences thêta, alpha et bêta au niveau du cortex préfrontal par rapport aux autres zones corticales. Pendant le sommeil lent profond, la puissance spectrale ne différait pas entre les groupes au niveau du cortex préfrontal. En revanche, par rapport aux bons dormeurs, les patients insomniaques avaient une puissance spectrale plus élevée dans la bande de fréquence bêta et une puissance spectrale moins élevée dans la bande de fréquence delta au niveau des autres aires corticales. Pendant le sommeil paradoxal, la puissance spectrale dans la bande de fréquence beta était plus faible chez les patients insomniaques par rapport aux bons dormeurs au niveau du cortex préfrontal. En revanche, aucune différence significative entre les groupes n’a été trouvée pour les autres aires corticales durant le sommeil paradoxal. Nos résultats montrent donc un profil d'activité corticale spécifique au niveau du cortex préfrontal chez les patients insomniaques par rapport aux bons dormeurs pendant toute la durée du sommeil. En outre, nos résultats suggèrent que les patients insomniaques pourraient avoir un dysfonctionnement dans la réactivation du système limbique pendant le sommeil paradoxal. Nos résultats donnent également des arguments supplémentaires en faveur d'une intégrité des mécanismes de protection du sommeil chez les patients insomniaques primaires.

Enfin, dans le Chapitre 7, nous avons discuté l'ensemble des études expérimentales de cette dissertation afin d'en tirer des conclusions générales sur les effets de la privation de sommeil et de l'insomnie primaire sur les performances diurnes et sur les fluctuations du signal EEG. Nous nous sommes particulièrement intéressés aux effets de la durée de la tâche. De plus, de nouvelles hypothèses ont été proposées en lien avec la pathophysiologie de l'insomnie primaire en tenant compte des résultats comportementaux et des résultats EEG. Nous avons également abordé la thématique de la prédiction des modifications de la performance de conduite en utilisant le signal EEG et proposé d'autres approches à cet effet. Enfin, nos résultats ont ouvert la voie pour de nouvelles hypothèses et de nouvelles expérimentations notamment en lien avec l'étude des bases neurales des déficits diurnes chez les patients insomniaques. De façon générale, cette thèse a permis de montrer que l'insomnie primaire est associée à une diminution de la vigilance ce qui pourrait avoir des effets néfastes sur les performances de conduite automobile notamment en condition de conduite monotone. En outre, les 
insomniaques primaires semblent souffrir d'un disfonctionnement du contrôle exécutif et de futures recherches en imagerie dans ce domaine sont nécessaires pour aller plus loin dans la compréhension de ces déficits. Des modifications particulières au niveau du cortex préfrontal lors du sommeil ont également été mises en lumière chez les insomniaques primaires. Au regard des effets de la privation de sommeil, nous avons montré que la somnolence qu'elle induit aggrave les effets de la durée de la tâche tant sur la performance de conduite que sur le signal EEG ce qui soulève la question de cet effet cumulatif dans la vie quotidienne et notamment en terme de sécurité routière. En somme, ce travail apporte des précisions nécessaires à la compréhension des conséquences néfastes de l'insomnie primaire et de la privation de sommeil totale et nos résultats ont permis d'élaborer de nouvelles perspectives de recherche dans le domaine de l'insomnie primaire. 



\section{Valorization addendum}

This addendum addresses the relevance and the impact of the results described in the current dissertation entitled "Performance and EEG measures in primary insomnia patients and during sleep deprivation in healthy volunteers". For this purpose, five topics are developed in relation to the social and economic relevance of our results, the target groups for whom our results are relevant, the activities and products that can derive from the work described in the current dissertation, the innovative aspect of our research, and finally, how ideas resulting from our work have been and will be implemented.

\section{Social and economic relevance}

Sleep disruption has become a major problem in Western society [1,2]. Among the different types of sleep disruption, we have considered sleep deprivation and chronic insomnia in the current dissertation. The latter is one of the most prevalent current health concerns [3,4]. Between 10 and $15 \%$ of adults in the general population are reported to have complaints of insomnia associated with complaints of daytime fatigue and cognitive impairments [5-7]. Insomnia is defined as a complaint of non-restorative and/or insufficient sleep associated with complaints of daytime consequences without comorbidities.

Both insomnia and sleep deprivation can lead to a range of adverse consequences on daytime performance, health, and productivity [8-14] that place a heavy economic burden on our society [14-17]. It has been estimated that, in the United States, insomnia was associated with $7.2 \%$ of all costly workplace accidents and errors [18], and the Sleep Health Foundation evaluated the indirect costs of sleep loss at 1.5 billion dollars in 2011 [14]. Cognitive and psychomotor dysfunction may hamper day-to-day operations such as driving a car. Indeed, insomnia has been indicated as an additional risk factor for traffic accidents [1921]. Epidemiological studies have also shown that sleep deprivation leads to increased sleepiness and increased risk of traffic accidents [22-25]. Furthermore, crash risk following sleep deprivation seems most pronounced during extended driving periods when loss of vigilance is highest [26-28]. 
Daytime performance of insomnia patients is described in Chapters 2, 3, and 4. The current thesis explored for the first time the driving performance of untreated primary insomnia patients during simulated and on-the-road driving. We have shown that insomnia patients, even without being treated for their sleep problems, could have a driving impairment in monotonous situations in simulated driving. In contrast, on-the-road driving investigation revealed that older insomnia patients are fit to drive on the highway, meaning that it is not a simple problem. In fact, compensation mechanisms can occur in older insomnia patients or the occurrence of frequent stimuli (as is the case in real highway driving compared to simulated monotonous driving) can preserve insomnia patients from driving impairments. In addition, our results showed that insomnia patients have a vigilance decrement that seems more prominent than in good sleepers during monotonous driving and difficulties in conflict resolution. Conflict resolution is part of the executive control process that includes a large range of abilities such as initiating, planning, setting goals, and resolving conflicts that are consistently useful in day-to-day life. Because we (and previous research) have shown that insomnia patients have difficulties in complex cognitive and monotonous driving tasks, these results are in line with complaints of daytime fatigue and cognitive impairments that are often expressed by insomnia patients in daily life activities such as those done at work and/or in their personal life. Daytime difficulties may also be related to economic issues. Firstly, by being less efficient at work and by having more absenteeism than people with good sleep, insomnia patients' sleep problems contribute to costs. Secondly, if insomnia patients are more likely to have driving accidents than good sleepers, related health care costs must also be higher.

Beside these daytime deficits, the sleep spectral investigation in insomnia patients described in Chapter 6 revealed a specific cortical activity pattern in the prefrontal cortex compared to other cortical areas (i.e. parietal, occipital, and central). As the prefrontal cortex mediates sleep physiology as well as high-order cognitive abilities, it may thus be a target for sleep and cognitive performance enhancement using electrical stimulation, as described in recent studies [29-32]. 
We also investigated the effects of sleep deprivation over time on real monotonous driving performance and cortical activity as assessed by electroencephalography (EEG) during driving. Laboratory investigations have shown that sleep deprivation exacerbates time-on-task effects (performance decrement over time). Results described in Chapter 5 revealed that the interaction between sleep deprivation and duration of driving is present in real conditions as well. In addition, sleep EEG results revealed that, in particular, the theta EEG power, which is related to sleepiness, was increased both after sleep deprivation and by duration of driving. The deleterious effects induced by the duration of driving were exacerbated by sleep deprivation compared to the normal sleep condition. During on-the-road driving, after sleep deprivation, the time-on-task effects found in both driving performance and cortical activity differ slightly from those found in simulated driving setting. Our results did not reveal any significant correlation between EEG and driving performance measures, similar to previous driving and EEG investigations. It appears therefore that EEG is not the ideal physiological measure for predicting driving performance.

\section{Target groups}

The results described in this dissertation are relevant to many groups including the academic community, insomnia patients, clinicians, governments, and companies.

Clinicians, and by extension governments, should be aware that, even if the daytime deficits of insomnia patients are difficult to describe using classic neuropsychological tests, these patients do have daytime difficulties as revealed in part by results from Chapters 2, 3, and 4, and insomnia patients' complaints need to be heard. Therefore, sleep clinicians and general practitioners are directly concerned by results described in this dissertation. Consequently, if the current results are taken into account in creating more comprehensive care in the management of insomnia patients' daytime and sleep problems, these patients will benefit from our results. In addition, the results described in this dissertation are relevant for governments, especially in the field of prevention because it gives scientific support to epidemiological reports about both the interaction of sleep 
deprivation and duration of driving. Complaints of driving difficulties in insomnia patients are also supported.

Ultimately, our results may help companies develop devices dedicated to the prevention of vigilance decrement during driving, both after sleep deprivation and in insomnia patients.

\section{Activities and products}

As a first step, our results on daytime difficulties in insomnia patients may help clinicians in the management of the cognitive difficulties of insomnia patients. Cognitive therapies in particular should focus on executive control and memory, which are the cognitive domains most affected in insomnia based on our report and those of previous studies.

The prevention of driving sleepy is still in development, and our results open new opportunities for preventing traffic accidents related to sleepiness. The results described in Chapter 5 together with results from previous driving studies suggest that results from simulator driving studies, especially EEG measurements, may not be completely representative of the sleepiness state of the driver. Therefore, results from simulator driving studies should not be used as a basis for preventing sleepy-based traffic accidents. By having better knowledge about the effects of sleep deprivation on actual driving particularly throughout the driving task and not an overall view, companies will be able to improve drowsy driver detection systems. Because our study in Chapter 5 and previous driving and EEG investigations led to inconsistent results about a clear relationship between EEG and driving measures, it seems that EEG is not the ideal physiological measure to predict driving performance. Thus, new combinations of physiological measures obtained on-board and able to predict driving impairment are needed. This could be done by forming collaborative relationship between companies and researchers who can interact and exchange their knowledge. This is a good opportunity to increase contribution of public research to private companies. Such development will lead to the creation of new systems that will involve researchers and start-ups as well as international automobile companies and will thus lead to patents. The development of high order statistical models to prevent hypo-vigilance will also 
involve statisticians who are accustomed to working with private companies. Therefore, various fields of research will contribute and interact to develop onboard systems for preventing sleepiness and hypo-vigilance.

Electrical stimulation to improve cognitive performance is becoming increasingly popular among researchers and companies. Based on tDCS research experiment results, companies have recently developed devices that apply small electrical currents directly onto the scalp, in order to stimulate neurons. This method has been shown to improve attention and cognitive abilities. Based on this knowledge, it is tempting to think about using these devices in insomnia patients or after sleep deprivation. One may imagine that we could use tDCS to reverse the deleterious effects of insomnia or sleep deprivation on daytime performance by using electrical stimulation over the prefrontal cortex, for example. However, although this method has been said to be non-invasive, this is not necessarily the case if non-experienced persons perform the stimulation. Indeed, in order to use tDCS properly, it is important to know how long to stimulate, at what time to stimulate, and what intensity to use. Unfortunately, this knowledge is only available to clinicians or researchers in the field who have already had experience with tDCS. Consequently, one can imagine that tDCS could be used to improve daytime cognitive performance in insomnia or after sleep deprivation, but such electrical stimulation needs to be done by clinicians from the field and cannot be done by the patient himself.

\section{Innovation}

The research described in this dissertation is innovative in various aspects. Firstly, to our knowledge, this is the first time that EEG measures and on-the-road driving performance have been investigated together in insomnia patients and after total sleep deprivation in comparison to a normal night of sleep in healthy volunteers. Such experiments give new insights into the physiological basis of driving when sleepy and of daytime deficits of insomnia patients, and may help to improve driving safety. Driving experiments have high ecological validity that is particularly true for on-the-road driving. Conducting on-the-road driving studies is a scientific and technical challenge that has been possible only because of the expertise of the 
Department of Neuropsychology and Psychopharmacology (Maastricht, The Netherlands) in this field of research.

Secondly, studying the driving performance of both healthy volunteers after sleep deprivation and insomnia patients in the same thesis is also innovative. Although surprising, most insomnia patients are not sleepy and have a reasonable amount of sleep. Therefore, sleep deprivation study results should not be extended to insomnia patients. The behavioral and EEG results described in this dissertation are in line with this assertion because we showed that driving performance particular as well as EEG measures over time differ between insomnia patients and healthy sleep deprived in participants.

Besides these general methodological aspects, the special feature of the statistical investigations of our results helped to highlight for the first time sleep and daytime characteristics of insomnia patients and healthy volunteers that were sleep deprived. In particular, in Chapter 6, we chose to consider all cortical areas while investigating sleep spectral analysis. This allowed us to find specific cortical activity patterns in insomnia patients that have not been highlighted before. In Chapters 3, 4, and 5, instead of considering driving performance and EEG measures in a global way, we investigated their fluctuations over time that yielded more in-depth results about driving performance deficits and cortical fluctuations over the driving task.

\section{Timeline and implementation}

As a first step, our results have already been presented at various national and international scientific and clinical conferences. In addition, the papers described in this dissertation have been published or are in revision in scientific journals that target both scientific and clinical areas of interest. Moreover, dissemination of information about our study in public journals (Inserm, Science et Santé, March $2015)$ and in scientific editorials $[33,34]$ has been achieved.

In the long-term, we hope that our findings will be used to pursue efforts in the education of the general public about the risks of drowsiness on the road. Until now, less attention has been given to insomnia patients on the road than 
sleep deprivation or drug use. The present results underline the fact that driving safety and accident prevention should be extended to patients with sleep disorders, particularly insomnia patients. Therefore, it would be wise to include insomnia in prevention campaigns for public education on driving risks. This also raises the question of whether or not insomnia patients are indeed dangerous on the road, and public debates should be organized on this topic, during French Sleep Day or French Science Day for example. One can also imagine that, as in the case of drunk-driving, an insomnia-driving association could be created in order to encourage research and development and dissemination of information about insomnia and driving. Previous campaigns of prevention have focused on sleepy/drowsy driving. However, insomnia patients are qualified as "hyperaroused" and thus not really sleepy. Even if the question remains to be elucidate regarding the neural correlates of driving impairment in insomnia, our results emphasize the fact that not only being sleepy can be dangerous on the road.

Cognitive therapies in insomnia patients should focus specifically on executive control and memory, which are the cognitive domains most affected in insomnia based on our report and those of previous studies. More generally, sleep centers may receive additional interest and provide the opportunity for their sleep physicians to spend more time on insomnia patients, as in the case for OSAS for example. Additional effort needs to be put into finding resources (financial and human) in order to better support insomnia patients and develop the use of nonpharmacological treatment to improve sleep and performance of daily activities. In France, consultations for insomnia patients are currently considered to be too time consuming and thus not cost-effective for hospitals, so less attention is given to insomnia compared to other sleep disorders which are easier to manage. Our governments should consider the long-term consequences and understand that, although not immediately profitable, the long-term benefits of better patient management and lessening of economic burden will outweigh the benefits of short-term solutions. Insomnia is not untreatable, and these patients should be shown more consideration. 


\section{References list}

[1] Bin YS, Marshall NS, Glozier N. Sleeping at the limits: the changing prevalence of short and long sleep durations in 10 countries. Am J Epidemiol 2013;177:82633. doi:10.1093/aje/kws308.

[2] Conti AA. Is our sleep time too short? Sleep deprivation as a pathophysiological condition of modern times. Acta Bio-Medica Atenei Parm 2012;83:234-5.

[3] Roth T, Roehrs T. Insomnia: epidemiology, characteristics, and consequences. Clin Cornerstone 2003;5:5-15.

[4] Buysse DJ. Insomnia. JAMA J Am Med Assoc 2013;309:706-16. doi:10.1001/jama.2013.193.

[5] Morin CM, LeBlanc M, Daley M, Gregoire JP, Mérette C. Epidemiology of insomnia: prevalence, self-help treatments, consultations, and determinants of help-seeking behaviors. Sleep Med 2006;7:123-30. doi:10.1016/j.sleep.2005.08.008.

[6] Ohayon MM. Epidemiology of insomnia: what we know and what we still need to learn. Sleep Med Rev 2002;6:97-111.

[7] Ohayon MM, Reynolds CF 3rd. Epidemiological and clinical relevance of insomnia diagnosis algorithms according to the DSM-IV and the International Classification of Sleep Disorders (ICSD). Sleep Med 2009;10:952-60. doi:10.1016/j.sleep.2009.07.008.

[8] Wang Q, Xi B, Liu M, Zhang Y, Fu M. Short sleep duration is associated with hypertension risk among adults: a systematic review and meta-analysis. Hypertens Res Off J Jpn Soc Hypertens 2012;35:1012-8. doi:10.1038/hr.2012.91.

[9] Cappuccio FP, D’Elia L, Strazzullo P, Miller MA. Sleep duration and all-cause mortality: a systematic review and meta-analysis of prospective studies. Sleep 2010;33:585-92.

[10] Baglioni C, Riemann D. Is chronic insomnia a precursor to major depression? Epidemiological and biological findings. Curr Psychiatry Rep 2012;14:511-8. doi:10.1007/s11920-012-0308-5.

[11] Daley M, Morin CM, LeBlanc M, Grégoire JP, Savard J, Baillargeon L. Insomnia and its relationship to health-care utilization, work absenteeism, productivity and accidents. Sleep Med 2009;10:427-38. doi:10.1016/j.sleep.2008.04.005. 
[12] Fortier-Brochu E, Beaulieu-Bonneau S, Ivers H, Morin CM. Relations between sleep, fatigue, and health-related quality of life in individuals with insomnia. J Psychosom Res 2010;69:475-83. doi:10.1016/j.jpsychores.2010.05.005.

[13] Hamblin JE. Insomnia: an ignored health problem. Prim Care 2007;34:659-74, viii. doi:10.1016/j.pop.2007.05.009.

[14] Hillman DR, Lack LC. Public health implications of sleep loss: the community burden. Med J Aust 2013;199:S7-10.

[15] Ozminkowski RJ, Wang S, Walsh JK. The direct and indirect costs of untreated insomnia in adults in the United States. Sleep 2007;30:263-73.

[16] Daley M, Morin CM, LeBlanc M, Grégoire J-P, Savard J. The economic burden of insomnia: direct and indirect costs for individuals with insomnia syndrome, insomnia symptoms, and good sleepers. Sleep 2009;32:55-64.

[17] Léger D, Bayon V. Societal costs of insomnia. Sleep Med Rev 2010;14:379-89. doi:10.1016/j.smrv.2010.01.003.

[18] Shahly V, Berglund PA, Coulouvrat C, Fitzgerald T, Hajak G, Roth T, et al. The associations of insomnia with costly workplace accidents and errors: results from the America Insomnia Survey. Arch Gen Psychiatry 2012;69:1054-63. doi:10.1001/archgenpsychiatry.2011.2188.

[19] Laugsand LE, Strand LB, Vatten LJ, Janszky I, Bjørngaard JH. Insomnia symptoms and risk for unintentional fatal injuries- The HUNT study. Sleep In press.

[20] Smolensky MH, Di Milia L, Ohayon MM, Philip P. Sleep disorders, medical conditions, and road accident risk. Accid Anal Prev 2011;43:533-48. doi:10.1016/j.aap.2009.12.004.

[21] Léger D, Bayon V, Ohayon MM, Philip P, Ement P, Metlaine A, et al. Insomnia and accidents: cross-sectional study (EQUINOX) on sleep-related home, work and car accidents in 5293 subjects with insomnia from 10 countries. J Sleep Res 2014;23:143-52. doi:10.1111/jsr.12104.

[22] Maia Q, Grandner MA, Findley J, Gurubhagavatula I. Short and long sleep duration and risk of drowsy driving and the role of subjective sleep insufficiency. Accid Anal Prev 2013;59:618-22. doi:10.1016/j.aap.2013.07.028. 
[23] Martiniuk ALC, Senserrick T, Lo S, Williamson A, Du W, Grunstein RR, et al. Sleep-deprived young drivers and the risk for crash: the DRIVE prospective cohort study. JAMA Pediatr 2013;167:647-55. doi:10.1001/jamapediatrics.2013.1429.

[24] Sagaspe P, Taillard J, Bayon V, Lagarde E, Moore N, Boussuge J, et al. Sleepiness, near-misses and driving accidents among a representative population of French drivers. J Sleep Res 2010;19:578-84. doi:10.1111/j.13652869.2009.00818.x.

[25] Stutts JC, Wilkins JW, Scott Osberg J, Vaughn BV. Driver risk factors for sleeprelated crashes. Accid Anal Prev 2003;35:321-31.

[26] Di Milia L, Kecklund G. The distribution of sleepiness, sleep and work hours during a long distance morning trip: a comparison between night- and non-night workers. Accid Anal Prev 2013;53:17-22. doi:10.1016/j.aap.2013.01.003.

[27] Di Milia L, Rogers NL, Åkerstedt T. Sleepiness, long distance commuting and night work as predictors of driving performance. PloS One 2012;7:e45856. doi:10.1371/journal.pone.0045856.

[28] Philip P, Taillard J, Guilleminault C, Quera Salva MA, Bioulac B, Ohayon M. Long distance driving and self-induced sleep deprivation among automobile drivers. Sleep 1999;22:475-80.

[29] Feusner JD, Madsen S, Moody TD, Bohon C, Hembacher E, Bookheimer SY, et al. Effects of cranial electrotherapy stimulation on resting state brain activity. Brain Behav 2012;2:211-20. doi:10.1002/brb3.45.

[30] Zheng X, Alsop DC, Schlaug G. Effects of transcranial direct current stimulation (tDCS) on human regional cerebral blood flow. NeuroImage 2011;58:26-33. doi:10.1016/j.neuroimage.2011.06.018.

[31] Brunoni AR, Nitsche MA, Bolognini N, Bikson M, Wagner T, Merabet L, et al. Clinical research with transcranial direct current stimulation (tDCS): Challenges and future directions. Brain Stimulat 2011. doi:10.1016/j.brs.2011.03.002.

[32] Jacobson L, Koslowsky M, Lavidor M. tDCS polarity effects in motor and cognitive domains: a meta-analytical review. Exp Brain Res Exp Hirnforsch Expérimentation Cérébrale 2012;216:1-10. doi:10.1007/s00221-011-28919. 
[33] Perlis ML, Roalf DR, Kloss JD. A commentary on the "Functioning of three attentional networks and vigilance in primary insomnia.” Sleep Med 2015. doi:10.1016/j.sleep.2015.08.010.

[34] Verster JC, Roth T. Insomnia and driving ability. Sleep 2014;37:1411-2. doi:10.5665/sleep.3984. 



\section{Remerciements/Acknowledgments}

Après presque 4 ans de thèse, voici enfin le moment tant attendu des remerciements... La recherche est un travail d'équipe, ces remerciements sont donc un peu longs... Because research is a team effort, these aknowledgments are quite long...

I would like to thank the members of the committee,

for accepting to read and judge the work that has been done during this thesis: Prof. Wim Riedel for accepting to be the Chair of this committee, Prof. Eus van Someren and Prof. Karel Brookhuis for accepting to review this work as external reviewers, dr. Arjan Blokland, dr. Géraldine Rauchs and Prof. Michel Boulouard as internal reviewers.

Chère Marie Laure, directrice de thèse,

merci de m'avoir fait confiance deux fois! La première fois pour un poste d’ingénieur et la seconde fois pour m’avoir proposé un financement de thèse. Merci aussi pour votre patience et vos conseils (notamment sur la rédaction... !) que je garde en mémoire autant que possible. L'apprentissage est loin d'être fini, je crois qu'il ne l'est jamais vraiment mais j'ai beaucoup appris pendant cette thèse.

Je tiens également à remercier les directeurs de l'équipe Eri27 devenue unité 1075, les professeurs Pierre Denise et Damien Davenne, pour m'avoir accueillie dans votre équipe ainsi que pour votre soutien tout au long de cette thèse.

Dear Jan (Ramaekers), my co-supervisor,

thank you for giving me the opportunity to do part of my $\mathrm{PhD}$ in Maastricht. Coming to Maastricht contributed to opening my mind and my knowledge. I learned a lot in Maastricht, not only from a scientific point of view.

Dear Annemiek,

thank you for being an unofficial supervisor. I really appreciated your constructive feedback and your thoroughness. 
Dear Eus,

thank you for giving me the opportunity to join your lab this year and to conduct this project. I have the chance to work in a very good environment and I could not have expected more for this first post doc. I hope this study will be successful. I would also like to thank the entire Sleep and Cognition team for its support during the last months of my $\mathrm{PhD}$. A special thanks to Jennifer Ramautar and Diederick Stoffers for your knowledge and help to begin the study; Yvon, Jessica, Marco, Valérie for your motivation to conduct the study with me. Thank you Michele for designing (so well!) the cover of this dissertation, Kim for your lastminute corrections...

Sebastien (Moussay),

merci d'avoir obtenu ce financement et de m'avoir fait confiance, tu as largement contribué à ce que ce post doctorat soit possible et tu as pour cela toute ma reconnaissance.

Merci également à Laurence Mouchnino et Martin Simoneau ainsi que l'équipe des Bases neurales de la sensori-motricité qui m’a accompagnée lors de mon stage de Master (Anahid, Jean Blouin, Khaled, Luc Tremblay, Alain Guillaume). Ce stage m’a permis de me passionner pour la recherche et cela n'aurait pas été possible sans l’influence de Laurence et Martin.

\section{Merci à toute l'équipe Eri27/U1075 à Caen.}

Chantal, merci pour vos connaissances, vos retours sur l'écriture qui ont permis d'améliorer notre article. Merci à toutes les personnes qui ont été présentes pour un mot, une douceur, un échange de bonne humeur ou par leur présence tout simplement. Annick et Yannick pour vos fabuleuses pauses gâteaux et recettes, on n’a pas conscience à quel point la thèse est formatrice en tous points ; Gwénaëlle, pour ton aide précieuse ta rigueur lors des protocoles; Daniel, pour ton aide informatique mais aussi ton humour et les pauses «clopes». Merci Antoine et Gaëlle pour votre bonne humeur chronique.

Merci aux doctorants passés et présents pour ces bons moments passés ensembles, je vous souhaite beaucoup de belles choses pour la suite: Michaël, 
Guillaume, Jean-Noël, Antoine (Junior), Chandrou, Kristell, Tristan, PierreAlexandre, Alice, Anne.

Je tiens également à remercier toutes les personnes que j’ai rencontré à l'Unité sommeil de Caen et qui ont contribué à ce travail de thèse : le Dr Françoise Bertran, le Dr Colette Couque, le Dr Jean-Noël Prevost, Carine, les infirmiers/ères de nuit et de jour pour m'avoir formé à la polysomnographie. Merci aussi à toutes les personnes qui ont participé aux protocoles de recherche même si je doute fort qu'elles ne lisent ce manuscrit.

Merci à Patrice (Clochon), Caroline (Harand) et Franck (Doidy) de l'Unité 1077 à Caen, j’ai eu la chance de pouvoir travailler et échanger avec vous sur le sommeil et l'EEG. Merci pour ces échanges et la bonne entente qui était présente lors de nos protocoles respectifs. Un vrai plus !

I switch in English to thank all my colleagues from the department of Neuropsychology and Psychopharmacology and friends that $I$ met in Maastricht:

Anke, Aimee, Zoé, Eliza, Judith, Anna, Sanae, Janelle, Luci, Anne, Stefan. (Bedankt) Thank you for being so patient with my English and for spending such a great time with me. Stefan, thank you for being such a nice coworker, studying sleep deprivation was a nice thing because of you. Anne, thank you for our nice bike-trips, the Netherlands is a flat country except around Maastricht... Thanks to both of you for accepting to be my paranymphs! Luci, thank you for being my roommate and friend, it was a great pleasure to meet you and Natalino.

Un petit mot particulier pour les collègues (ou conjoint de) devenus des amis et qui m’ont aussi soutenu durant cette thèse, Audrey Mondou, Marion Marais, Nicolas Margas, Jan et Oana. Jan, merci pour tes conseils « stats », tes conseils de chercheur en général, tu m’as fait confiance et tu m’as beaucoup appris !

Marie-Laure, Marie-Sophie, Lucile et Marion, «mes» morues. Merci pour ces moments passés ensemble et votre soutien pendant ces années de thèse. La thèse apporte aussi beaucoup sur le plan personnel. 
Et les derniers mots pour la famille qui me soutient depuis longtemps.

Mon Lilou, merci d'avoir été là toutes ces années et plus encore, merci de nous avoir tant donné. Merci aussi à toute la famille «Dindelli », en particulier Alberto et Sylvette pour votre présence familiale durant cette thèse.

Mon Papa, Françoise, vous m'avez tant appris, de par votre expérience. Papa tu as un bagage personnel incroyable de diversité et tu as su nous le transmettre à ta façon... Merci à tous les deux pour votre présence.

Ma Mum, merci pour....tout....ton soutien, ton amour, ta confiance, ta présence, tu as toujours su trouver les mots et les gestes pour me (re)motiver et affronter les difficultés des études universitaires (mais pas que), merci.

Sœur, tu m’as apporté (et m’apporte toujours) la sagesse d'une personne qui a un autre regard sur le monde, tu m'as permis d'avancer dans l'aventure de la thèse d'une façon différente et tu as su t'intéresser à mon travail, continues ton chemin, garde le cap, tu es super comme cela, un peu hors du lot.

Nico, Titouan, il faut croire que la thèse ne se vit pas seule mais à 2 , voire à 3 ! Vous avez supporté mes sautes d'humeurs et mes exclamations de joies, les derniers mois d'écriture et un déménagement aux Pays Bas. Et tout cela avec le sourire, MERCI! 


\section{Curriculum Vitae}

Joy Perrier was born on the $27^{\text {th }}$ of July 1987 in Marseille (France). In 2005, she obtained her High school diploma with a scientific speciality in Luynes (France Bouches du Rhône). Then, she studied physiology, biochemistry and neuroscience during her Bachelor at the University of Aix-Marseille between 2006 and 2008. After receiving her Master's degree in Cognitive Neuroscience with honors in 2010, she worked for a year as a researcher on the European project DRUID (Driving Under the Influence of Drugs) within the host team ERI27 INSERM CHU Caen. Following this work as an engineer, she continued to work in research starting a $\mathrm{PhD}$ in 2011 on insomnia, psychotropic and driving in the Unit 1075 COMETE INSERM at the University of Caen (France). This thesis is a joint $\mathrm{PhD}$ with the University of Maastricht where Joy Perrier was invited for one year (2012-2013) in the Department of Neuropsychology and Psychopharmacology to work on a project to study the effects of sleep deprivation on driving performance and EEG. She is currently working as a post doctorate since January 2015 at the University of Amsterdam in the laboratory Sleep and Cognition (Nederlands Institute for Neuroscience) to study the mechanisms associated with cognitive deficits in primary insomnia via fMRI coupled with EEG. 
Joy Perrier est née le 27 juillet 1987 à Marseille (France). En 2005, elle obtient son baccalauréat général spécialité scientifique à Luynes (France - Bouches du Rhône). Elle a ensuite étudié la physiologie, la biochimie et les neurosciences lors de sa licence à l'université d'Aix-Marseille entre 2006 et 2008. Après avoir obtenu son Master recherche mention Assez bien en neurosciences cognitives en 2010, elle a travaillé pendant un an en tant que chargée d'études sur le projet européen DRUID (DRiving Under the Influence of Drugs) au sein de l'équipe d'accueil Inserm ERI27 au CHU de Caen. A la suite de ce travail d'ingénieur, elle poursuit son travail dans la recherche en commençant une thèse en 2011 sur l'insomnie, les psychotropes et la conduite automobile au sein de l'unité Inserm 1075 COMETE au CHU de Caen (France). Cette thèse devient une thèse en cotutelle avec l'université de Maastricht où Joy Perrier est invitée pendant un an (2012-2013) dans le laboratoire de Neuropsychology and Psychopharmacology pour travailler sur un projet visant à étudier les effets de la privation de sommeil sur les performances de conduite et l'EEG. Elle effectue actuellement un post doctorat depuis le mois de janvier 2015 à l'université d'Amsterdam, au sein du laboratoire Sleep and Cognition (Institut néerlandais de recherche en neurosciences), afin d'étudier, via l'IRMf couplé à l'EEG, les mécanismes associés aux déficits cognitifs dans l'insomnie primaire. 


\section{Publications list}

Perrier, J., Clochon, P., Bertran, F., Couque, C., Bulla, J., Denise, P., Bocca, M.L. (2015). Specific EEG sleep pattern in the prefrontal cortex in primary insomnia. PLoS One, 1O(1), e0116864.

Perrier, J., Bertran, F., Marie, S., Couque, C., Bulla, J., Denise, P., Bocca, M.L. (2014). Impaired driving performance associated with effect of time duration in patients with primary insomnia. Sleep, 37(9), 1565-1573.

Perrier J., Leufkens TRM, Bulla J., Jongen S., Vuurman E., Bocca M.L., Ramaekers J.G., Vermeeren A. (2015). Electroencephalography during on-theroad driving in older untreated insomnia patients and normal sleepers. Biological Psychology, 109, 20-28.

Perrier J., Chavoix C., Marie S., Bocca M.L. (Accepted). Functionning of the three attentional networks and vigilance in primary insomnia. Sleep Medicine.

Jongen, S., Perrier, J., Vuurman, E. F., Ramaekers, J. G., Vermeeren, A. (2015). Sensitivity and Validity of Psychometric Tests for Assessing Driving Impairment: Effects of Sleep Deprivation. PloS One, 10(2), e0117045.

Saradjian, A. H., Tremblay, L., Perrier, J., Blouin, J., Mouchnino, L. (2013). Cortical facilitation of proprioceptive inputs related to gravitational balance constraints during step preparation. Journal of Neurophysiology, 110(2), 397407.

Mouchnino L., Fontan A., Tandonnet C., Perrier J., Saradjian A., Blouin J., Simoneau M. (2015). Facilitation of cutaneous inputs during the planning phase of gait initiation. Journal of Neurophysiology, 114(1), 301-308.

\section{Submitted}

Perrier J., Jongen S., Vuurman E., Bocca M.L., Ramaekers J.G., Vermeeren A. (Submitted). Sleepiness linked to sleep deprivation during on-the-road driving: a behavioral and physiogical investigation of time on task effects. 
208 Publications list

In preparation

Perrier J., Jongen S., Vuurman E., Bocca M.L., Ramaekers J.G., Vermeeren A.

(In preparation). The effects of sleep deprivation on attention and vigilance: an EEG investigation. 